\title{
MMP for co-rank one foliations on threefolds
}

\section{Paolo Cascini $^{1}$ - Calum Spicer ${ }^{1}$}

Received: 11 August 2018 / Accepted: 28 January 2021 / Published online: 4 March 2021

(C) The Author(s) 2021

\begin{abstract}
We prove existence of flips, special termination, the base point free theorem and, in the case of log general type, the existence of minimal models for F-dlt foliated pairs of co-rank one on a $\mathbb{Q}$-factorial projective threefold. As applications, we show the existence of F-dlt modifications and F-terminalisations for foliated pairs and we show that foliations with canonical or F-dlt singularities admit non-dicritical singularities. Finally, we show abundance in the case of numerically trivial foliated pairs.
\end{abstract}

Mathematics Subject Classification 14E30 - 37F75

\section{Contents}

1 Introduction . . . . . . . . . . . . . . . . . . . . . 604

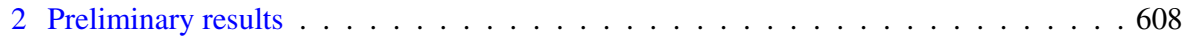

3 F-dlt foliated pairs and basic adjunction type results . . . . . . . . . . . 623

4 Approximating formal divisors . . . . . . . . . . . . . . . . . . . . 643

5 Approximating formal separatrices . . . . . . . . . . . . . . . . . 646

6 Constructing the flip . . . . . . . . . . . . . . . . . . . . 648

7 Special termination . . . . . . . . . . . . . . . . . . . 654

8 Existence of F-dlt modifications . . . . . . . . . . . . . . . . . . . 659

$凶$ Paolo Cascini

p.cascini@imperial.ac.uk

Calum Spicer

calum.spicer@imperial.ac.uk

1 Department of Mathematics, Imperial College London, 180 Queen's Gate, London SW7 2AZ, UK 


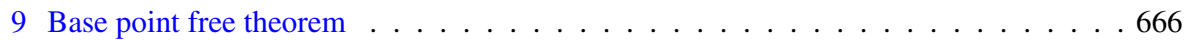

10 Minimal model program with scaling . . . . . . . . . . . . . . . . . . 669

11 Existence of F-terminalisations . . . . . . . . . . . . . . . . . . 673

12 Abundance for $c_{1}\left(K_{\mathcal{F}}+\Delta\right)=0 \ldots \ldots \ldots 676 \ldots \ldots$

References . . . . . . . . . . . . . . . . . . . . 688

\section{Introduction}

The (classical) Minimal Model Program predicts that a complex projective manifold is either uniruled or it admits a minimal model, i.e. it is birational to a (possibly singular) projective variety with nef canonical divisor. Although this is still an open problem, many important cases of the program have been carried out successfully, e.g. in the case of varieties of dimension at most three and for varieties of general type. After the work of McQuillan [31] we expect a similar picture to hold in the theory of birational geometry of foliations (see also [9,32]). More specifically, assuming that $X$ is a normal complex projective variety and $\mathcal{F}$ is a foliation with mild singularities, it is conjectured that either $\mathcal{F}$ is uniruled, i.e. $X$ is covered by rational curves which are tangent to $\mathcal{F}$, or $\mathcal{F}$ admits a minimal model, i.e. $X$ is birational to a projective variety $Y$ such that the transformed foliation on $Y$ (cf. Sect. 2.3) admits a nef canonical divisor (cf. Sect. 2.1). Many of the main goals of the program were carried out successfully in the case of rank one foliations (cf. [30,31]) and, in any rank, it is expected to follow the main steps of Mori's program.

The goal of this paper is to show the existence of flips (cf. Sect. 2.6) for foliations of co-rank one on a complex projective threefold and present several applications, under some natural assumptions on the singularities.

\subsection{Statement of main results}

In [41] it was shown that given a foliated pair $(\mathcal{F}, \Delta)$, with some mild assumption on the singularities, and given a $\left(K_{\mathcal{F}}+\Delta\right)$-negative extremal ray $R$, there is a morphism $\phi_{R}: X \rightarrow Y$, in the category of algebraic spaces, contracting only those curves $C$ such that $[C] \in R$. Projectivity of $Y$ and the existence of flips were shown in some special cases, but not in the generality needed to run the MMP.

Our first main result is to show in greater generality that if $\phi_{R}$ is a flipping contraction then the flip exists:

Theorem 1.1 (= Theorem 6.4 + Theorem 11.3) Let $\mathcal{F}$ be a co-rank one foliation on a $\mathbb{Q}$-factorial projective threefold $X$ and let $\Delta \geq 0$ such that $(\mathcal{F}, \Delta)$ is $F$-dlt. Let $\phi: X \rightarrow Y$ be a $\left(K_{\mathcal{F}}+\Delta\right)$-flipping contraction.

Then the $\left(K_{\mathcal{F}}+\Delta\right)$-flip exists. 
Notice that F-dlt foliated pairs play the same role as dlt log pairs in the classical MMP (see Definition 3.6 for a precise definition).

Next, we turn to the question of constructing a minimal model of a foliated pair $(\mathcal{F}, \Delta)$. As in Mori's program, existence of minimal models would follow if one could show that any sequence of flips terminates. We are unable to show termination in complete generality, but we are able to show a weaker version of termination, i.e., termination of flips with scaling, which suffices to show that minimal models exist in several cases of interest:

Theorem 1.2 (= Theorem $10.3+$ Theorem 11.3) Let $\mathcal{F}$ be a co-rank one foliation on a $\mathbb{Q}$-factorial projective threefold $X$. Let $\Delta=A+B$ be a $\mathbb{Q}$ divisor such that $(\mathcal{F}, \Delta)$ is a $F$-dlt pair, $A \geq 0$ is an ample $\mathbb{Q}$-divisor and $B \geq 0$. Assume that there exists a $\mathbb{Q}$-divisor $D \geq 0$ such that $K_{\mathcal{F}}+\Delta \sim_{\mathbb{Q}} D$.

Then $(\mathcal{F}, \Delta)$ admits a minimal model.

See Sect. 10 for a precise definition of a minimal model.

It is important to observe that Theorems 1.1 and 1.2 make no assumptions on the singularities of $X$ other than $\mathbb{Q}$-factoriality. However, as we will see by Theorem 11.3, the output of the MMP (and the intermediary steps of the MMP more generally) will be $\mathbb{Q}$-factorial varieties with klt singularities. Notice also that we first prove Theorems 1.1 and 1.2 under the assumption that the foliation has non-dicritical singularities (cf. Definition 2.10) but we later prove that, if $(\mathcal{F}, \Delta)$ is an F-dlt foliated pair then $\mathcal{F}$ admits non-dicritical singularities (cf. Theorem 11.3).

Along the way to proving the termination of flips with scaling, we prove the following basepoint free theorem for foliations which we expect will be of interest. Observe that if $\mathcal{F}$ is a rank one surface foliation with $K_{\mathcal{F}}$ nef and big then $K_{\mathcal{F}}$ is in general not semi-ample, see for instance [31, Corollary IV.2.3]. On the other hand, we prove the following:

Theorem 1.3 (= Theorem 9.4) Let $\mathcal{F}$ be a co-rank one foliation on a $\mathbb{Q}$ factorial projective variety $X$ of dimension at most three. Let $\Delta$ be a $\mathbb{Q}$-divisor such that $(\mathcal{F}, \Delta)$ is a $F$-dlt pair. Let $A \geq 0$ and $B \geq 0$ be $\mathbb{Q}$-divisors such that $\Delta=A+B$ and $A$ is ample. Assume that $K_{\mathcal{F}}+\Delta$ is nef.

Then $K_{\mathcal{F}}+\Delta$ is semi-ample.

Note that our result is in some sense optimal. Indeed, contrary to the case of varieties, if $\mathcal{F}$ is a foliation such that $K_{\mathcal{F}}$ is big and nef, then we cannot choose in general a divisor $\Delta \geq 0$ such that $\Delta \sim_{\mathbb{Q}} \epsilon K_{\mathcal{F}}$ for some $\epsilon>0$ and $(\mathcal{F}, \Delta)$ is F-dlt, as some of the components of $\Delta$ might be $\mathcal{F}$-invariant.

\subsection{Application to F-dlt modifications and F-terminalisations}

In the study of the birational geometry of varieties, dlt modifications and terminalisations have proven to be very useful tools. The existence of these 
modifications follows from the MMP for varieties. We prove foliated analogues of these modifications as a consequence of our results on the foliated MMP (see Sect. 2.2 for the definition of the number $\epsilon$ and Sect. 2.3 for the notion of the transform of a foliation under a birational map):

Theorem 1.4 (Existence of F-dlt modifications, = Theorem 8.1) Let $\mathcal{F}$ be $a$ co-rank one foliation on a normal projective variety $X$ of dimension at most three. Let $(\mathcal{F}, \Delta)$ be a foliated pair.

Then there exists a birational morphism $\pi: Y \rightarrow X$ such that if $\mathcal{G}$ is the transformed foliation on $Y$ then $\left(\mathcal{G}, \pi_{*}^{-1} \Delta+\sum \epsilon\left(E_{i}\right) E_{i}\right)$ is $F$-dlt where the sum is taken over all the $\pi$-exceptional divisors and

$$
\left(K_{\mathcal{G}}+\pi_{*}^{-1} \Delta+\sum \epsilon\left(E_{i}\right) E_{i}\right)+G=\pi^{*}\left(K_{\mathcal{F}}+\Delta\right),
$$

where $G \geq 0$.

In particular, if $(\mathcal{F}, \Delta)$ is lc then $\pi$ only extracts divisors of discrepancy $=-\epsilon\left(E_{i}\right)$.

Furthermore, we may choose $(Y, \mathcal{G})$ so that

1. if $Z$ is an lc centre of $\left(\mathcal{G}, \pi_{*}^{-1} \Delta+\sum \epsilon\left(E_{i}\right) E_{i}\right)$ then $Z$ is contained in a codimension one lc centre of $\left(\mathcal{G}, \pi_{*}^{-1} \Delta+\sum \epsilon\left(E_{i}\right) E_{i}\right)$,

2. $Y$ is $\mathbb{Q}$-factorial and

3. $Y$ is klt.

Theorem 1.5 (Existence of F-terminalisations, = Theorem 11.1) Let $\mathcal{F}$ be a co-rank one foliation on a normal projective variety $X$ of dimension at most three.

Then there exists a birational morphism $\pi: Y \rightarrow X$ such that

1. if $\mathcal{G}$ is the transformed foliation on $Y$, then $\mathcal{G}$ is $F$-dlt, canonical and terminal along Sing $Y$,

2. $Y$ is klt and $\mathbb{Q}$-factorial and

3. $K_{\mathcal{G}}+E=\pi^{*} K_{\mathcal{F}}$ where $E \geq 0$.

Using similar ideas we are also able to prove the following:

Theorem 1.6 (= Theorem 11.3) Let $(\mathcal{F}, \Delta)$ be a foliated pair on a normal projective threefold $X$. Suppose that $(\mathcal{F}, \Delta)$ is canonical.

Then $\mathcal{F}$ has non-dicritical singularities (cf. Definition 2.10).

Observe that we do not require the smoothness of $X$. We expect that this result will be useful in the study of foliation singularities.

\subsection{Application to foliation abundance}

It is a direct consequence of [29, Theorem 2] that if $X$ is a projective manifold with $\mathcal{F}$ a co-rank one foliation with canonical singularities and $c_{1}\left(K_{\mathcal{F}}\right)=$ 
0 then $K_{\mathcal{F}}$ is torsion. When $X$ is a threefold we extend this result to the $\log$ situation where we consider $\mathcal{F}$ together with a boundary $\Delta$, as well as weakening the hypotheses on the singularities.

Theorem 1.7 (= Theorem 12.1) Let $\mathcal{F}$ be a co-rank one foliation on a projective threefold $X$. Let $(\mathcal{F}, \Delta)$ be a foliated pair with log canonical foliation singularities. Suppose that $c_{1}\left(K_{\mathcal{F}}+\Delta\right)=0$.

Then $\kappa\left(K_{\mathcal{F}}+\Delta\right)=0$.

\subsection{Sketch of the proof}

We first give a rough outline of our approach to the existence of flips. Let us focus on a special case first: suppose $X$ is a smooth threefold and $\mathcal{F}$ is the foliation induced by a fibration $f: X \rightarrow B$ onto a curve $B$ and with simple normal crossing fibres. It is easy to compute that $K_{\mathcal{F}}=K_{X / B}-\sum\left(r_{i}-1\right) F_{i}$ where the sum runs over the components of fibres with multiplicity $r_{i}$.

Let $C \subset X$ be a $K_{\mathcal{F}}$-flipping curve (cf. Sect. 2.6). It was shown in [41] that $C$ is tangent to $\mathcal{F}$ (cf. Definition 2.12). Let $T=f^{*}(f(C))_{\text {red }}$ and let $S$ be a component of $T$ containing $C$. Note that $T$ is the largest connected $\mathcal{F}$-invariant divisor containing $C$ (cf. Sect. 2.2). A direct computation shows that $\left.K_{\mathcal{F}}\right|_{S}=$ $\left.\left(K_{X}+T\right)\right|_{S}$ and, in particular, it follows that $\left(K_{X}+T\right) \cdot C=K_{\mathcal{F}} \cdot C<0$ and since $(X, T)$ is log canonical it follows that the $K_{\mathcal{F}}$-flip can be realised as a $\log$ flip in the classical MMP.

In general, if $X$ is a normal threefold and $\mathcal{F}$ is a co-rank one foliation on $X$ with mild singularities and which admits a flipping contraction (cf. Sect. 2.6), we want to realise the $K_{\mathcal{F}}$-flip as a $\left(K_{X}+\sum S_{i}\right)$-flip where the $S_{i}$ are all the $\mathcal{F}$-invariant divisors meeting $C$. There are two technical challenges here. The first is that the $S_{i}$ are not necessarily algebraic divisors, and if $C \subset \operatorname{sing}(\mathcal{F})$, they might not even be defined analytically locally around $C$ : instead they might only exist as formal divisors in the formal completion of $X$ along $C$. The second challenge is to control the singularities of the pair $\left(X, \sum S_{i}\right)$.

To handle the first challenge we develop an extension of the classical MMP to the formal setting. We adapt some approximation results of Artin/Elkik to show that we can approximate the $S_{i}$ by algebraic divisors $S_{i}^{\prime}$ on an étale neighbourhood $U$ of $C$ in the sense that $S_{i}=S_{i}^{\prime}$ on some infinitesimal neighbourhood of $C$. By choosing a sufficiently close approximation, it follows that the $\left(K_{U}+\sum S_{i}^{\prime}\right)$-flip coincides with the $\left(K_{X}+\sum S_{i}\right)$-flip. We emphasise that we are only approximating the $S_{i}$ and we are not approximating the foliation $\mathcal{F}$, i.e., the $S_{i}^{\prime}$ are not necessarily invariant divisors of some other foliation $\mathcal{F}^{\prime}$. Indeed, it is well known that it is not in general possible to approximate a foliation with non-convergent separatrices by one with convergent separatrices (see Sects. 4, 5). 
Controlling the singularities of $\left(X, \sum S_{i}\right)$ is done by way of several results which provide bounds between the singularities of $\mathcal{F}$ and those of $\left(X, \sum S_{i}\right)$. There is some additional difficulty arising from the fact that the $S_{i}$ are not necessarily $\mathbb{Q}$-Cartier (even if $X$ is $\mathbb{Q}$-factorial). This can be handled by somewhat standard arguments from the classical MMP (see Sect. 6).

The remaining results on the MMP for foliations of co-rank one on a threefold (i.e., special termination, basepoint free theorem, termination of flips with scaling, existence of F-dlt modifications, etc.) are mostly a consequence of direct translations of standard ideas and strategies from the classical MMP to the foliated setting. Nevertheless there are some intriguing issues which arise and which are unique to the foliated setting (e.g., foliations may admit infinitely many lc centres).

Finally, our abundance type result for foliations with $c_{1}\left(K_{\mathcal{F}}\right)=0$ is a consequence of a rather lengthy and delicate case by case analysis. Some central ingredients are Touzet's results on foliations with pseudo-effective conormal bundle and a careful analysis of families of surfaces foliations with trivial canonical class (see Sect. 12).

\section{Preliminary results}

We work over the field of complex numbers $\mathbb{C}$. Throughout the paper, a variety is a complex analytic space.

Let $X$ be a normal variety and let $V \subset X$ be a closed subvariety. Let $\hat{X}$ be the formal completion of $X$ along $V$. A formal divisor $D$ on $\hat{X}$ is a union of distinct integral formal subschemes of pure codimension one. Let $I_{D}$ be the ideal sheaf of $D$. We say that $D$ is $\mathbb{Q}$-Cartier if $\left(I_{D}^{\otimes n}\right)^{* *}$ is locally of the form $f \cdot \mathcal{O}_{\widehat{X}}$ where $f$ is a local section of $\mathcal{O}_{\widehat{X}}$ and $n$ is a positive integer. We say that $D$ does not contain $V$ in its support if $f$ does not vanish along $V$. Note that, in this case, if we denote by $v: V^{v} \rightarrow V$ the normalisaton of $V$, we can define the pull-back of $D$ to $V^{v}$ as a $\mathbb{Q}$-Cartier divisor on $V^{v}$.

Let $X$ be a smooth variety of dimension $n$ and let $D$ be a reduced divisor, we say that $D$ is normal crossing, or that $(X, D)$ is a normal crossing pair, if, for each closed point $x \in D$, there exist local formal coordinates $x_{1}, \ldots, x_{n}$ such that $D$ is defined by $\left\{x_{1} \cdots x_{r}=0\right\}$ for some $1 \leq r \leq n$. Note that this definition works equally well even if $\hat{X}$ is the formal completion of a smooth variety $X$ along a closed subvariety and $D \subset \hat{X}$ is a formal divisor. We say that a divisor $D$ on a smooth variety $X$ is simple normal crossing if it is normal crossing and every irreducible component of $D$ is smooth.

Given a normal variety $X$, we denote by $\Omega_{X}^{1}$ its sheaf of Kähler differentials and, by $T_{X}:=\left(\Omega_{X}^{1}\right)^{*}$ its tangent sheaf. For any positive integer $p$, we denote $\Omega_{X}^{[p]}:=\left(\Omega_{X}^{p}\right)^{* *}$. 
Given a $\mathbb{Q}$-divisor $\Delta$ on a normal variety $X$, we write $\lfloor\Delta\rfloor$ for the round-down of $\Delta$ and $\{\Delta\}$ for the fractional part of $\Delta$, i.e. $\{\Delta\}=\Delta-\lfloor\Delta\rfloor$. A $\mathbb{Q}$-factorial variety is a normal variety $X$ on which every divisor is $\mathbb{Q}$-Cartier. A birational map $f: X \rightarrow Y$ between normal varieties is a birational contraction if $f^{-1}$ does not contract any divisor.

We refer to [25, Sect. 2.3] for the classical definitions of singularities (e.g. klt, $\log$ canonical, etc.) appearing in the minimal model program. In particular, a log pair $(X, \Delta)$ consists of a normal variety $X$ and a $\mathbb{Q}$-divisor $\Delta$ with coefficients in $(0,1]$ and such that $K_{X}+\Delta$ is $\mathbb{R}$-Cartier. Note that if the coefficients of $\Delta$ are rational, then $K_{X}+\Delta$ is $\mathbb{Q}$-Cartier. If $S$ is an irreducible component of $\lfloor\Delta\rfloor$, and $v: S^{\nu} \rightarrow S$ is its normalisation, then we may write

$$
\left.\left(K_{X}+\Delta\right)\right|_{S^{\nu}}=K_{S^{\nu}}+\Theta,
$$

where $\Theta$ is an effective $\mathbb{R}$-divisor on $S^{\nu}$ called the different of $(X, \Delta)$ with respect to $S$ (cf. [28, (4.2.9)]).

Let $(X, \Delta)$ be a log pair, let $S \subset X$ be a prime divisor contained in the support of $\lfloor\Delta\rfloor$ and let $\widehat{X}$ be the formal completion of $X$ along $S$. Assume that $T$ is a $\mathbb{Q}$-Cartier formal divisor on $\widehat{X}$ which does not contain $S$ in its support. Then we define the different of $(\widehat{X}, \Delta+T)$ with respect to $S$, as $\Theta+v^{*} T$, where $\Theta$ is the different of $(X, \Delta)$ with respect to $S$.

We say that a normal variety $X$ is potentially klt if there exists a $\mathbb{Q}$-divisor $\Delta \geq 0$ such that $(X, \Delta)$ is klt. We say that a normal variety $X$ is étale locally potentially klt if for all $x \in X$ there is an étale neighborhood $U$ of $x$ such that $U$ is potentially klt.

\subsection{Basic definitions}

A foliation on a normal variety $X$ is a coherent subsheaf $\mathcal{F} \subset T_{X}$ such that

1. $\mathcal{F}$ is saturated, i.e. $T_{X} / \mathcal{F}$ is torsion free, and

2. $\mathcal{F}$ is closed under Lie bracket.

The rank of $\mathcal{F}$ is its rank as a sheaf. Its co-rank is its co-rank as a subsheaf of $T_{X}$.

Let $X$ be a normal variety and let $\mathcal{F}$ be a rank $r$ foliation on $X$. We can associate to $\mathcal{F}$ a morphism

$$
\phi: \Omega_{X}^{[r]} \rightarrow \mathcal{O}_{X}\left(K_{\mathcal{F}}\right)
$$

defined by taking the double dual of the $r$-wedge product of the map $\Omega_{X}^{[1]} \rightarrow$ $\mathcal{F}^{*}$, induced by the inclusion $\mathcal{F} \subset T_{X}$. This yields a map

$$
\phi^{\prime}:\left(\Omega_{X}^{[r]} \otimes \mathcal{O}_{X}\left(-K_{\mathcal{F}}\right)\right)^{* *} \rightarrow \mathcal{O}_{X}
$$


and we define the singular locus of $\mathcal{F}$, denoted by $\operatorname{Sing} \mathcal{F}$, to be the cosupport of the image of $\phi^{\prime}$. A canonical divisor of $\mathcal{F}$ is a divisor $K_{\mathcal{F}}$ such that $\mathcal{O}_{X}\left(-K_{\mathcal{F}}\right) \cong \operatorname{det}(\mathcal{F})$. We define the normal sheaf of $\mathcal{F}$ as $\mathcal{N}_{\mathcal{F}}:=\left(T_{X} / \mathcal{F}\right)^{* *}$. The conormal sheaf $\mathcal{N}_{\mathcal{F}}^{*}$ of $\mathcal{F}$ is the dual of $\mathcal{N}_{\mathcal{F}}$. If $\mathcal{F}$ is a foliation of co-rank one then, by abuse of notation, we denote by $N_{\mathcal{F}}^{*}$ a divisor associated to $\mathcal{N}_{\mathcal{F}}^{*}$.

\subsection{Invariant subvarieties}

Let $X$ be a normal variety and let $\mathcal{F}$ be a rank $r$ foliation on $X$. Let $S \subset X$ be a subvariety. Then $S$ is said to be $\mathcal{F}$-invariant, or invariant by $\mathcal{F}$, if for any open subset $U \subset X$ and any section $\partial \in H^{0}(U, \mathcal{F})$, we have that

$$
\partial\left(\mathcal{I}_{S \cap U}\right) \subset \mathcal{I}_{S \cap U}
$$

where $\mathcal{I}_{S \cap U}$ denotes the ideal sheaf of $S \cap U$. If $D \subset X$ is a prime divisor then we define $\epsilon(D)=1$ if $D$ is not $\mathcal{F}$-invariant and $\epsilon(D)=0$ if it is $\mathcal{F}$-invariant.

Let $X$ be a normal variety and let $\mathcal{F}$ be a foliation on $X$. Let $\widehat{X}$ be the formal completion of $X$ along a proper closed subvariety $V \subset X$. We say that a formal subvariety $S \subset \widehat{X}$ is a formal $\mathcal{F}$-invariant divisor if it is a formal divisor which is $\widehat{\mathcal{F}}$-invariant, where $\widehat{\mathcal{F}}$ is the restriction of $\mathcal{F}$ to $\widehat{X}$.

\subsection{Transform of a foliation under a rational map}

Let $X$ be a normal variety and let $\mathcal{F}$ be a foliation on $X$. Let $\phi: Y \rightarrow X$ be a dominant map and assume that there exist smooth open subsets $U \subset X$ and $V \subset Y$ such that the restriction $\left.\phi\right|_{V}: V \rightarrow U$ is a morphism.

Let $\mathcal{F}_{U}$ denote the restriction of $\mathcal{F}$ to $U$. Then the morphism $\mathcal{N}_{\mathcal{F}_{U}}^{*} \rightarrow \Omega_{U}^{1}$ induces a morphism $\left(\left.\phi\right|_{V}\right)^{*} \mathcal{N}_{\mathcal{F}_{U}} \rightarrow \Omega_{V}^{1}$ and, therefore, a foliation $\mathcal{G}_{V}$ on $V$. We may extend $\mathcal{G}_{V}$ to a foliation on all of $Y$. Indeed, we may take $\mathcal{G}$ to be the saturated subsheaf of $T_{X}$ whose restriction to $V$ is $\mathcal{G}_{V}$. It is easy to see that $\mathcal{G}$ is closed under Lie bracket, since it is closed under Lie bracket over a dense open subset. We will refer to $\mathcal{G}$ as the induced foliation on $Y$ by $\phi$. If $\phi: Y \rightarrow X$ is a morphism, then the induced foliation is called the pulled back foliation and we denote it by $\phi^{-1} \mathcal{G}$. If $f: X \rightarrow X^{\prime}$ is a birational map, then the induced foliation on $X^{\prime}$ by $f^{-1}$ is called the transformed foliation of $\mathcal{F}$ by $f$ and we will denote it by $f_{*} \mathcal{F}$. 


\subsection{Foliation singularities}

Frequently, in birational geometry, it is useful to consider pairs $(X, \Delta)$ where $X$ is a normal variety and $\Delta$ is a $\mathbb{Q}$-Weil divisor such that $K_{X}+\Delta$ is $\mathbb{Q}$-Cartier. By analogy, we define:

Definition 2.1 Let $X$ be a normal variety. A foliated pair $(\mathcal{F}, \Delta)$ on $X$ consists of a foliation $\mathcal{F}$ on $X$ and a $\mathbb{R}$-divisor divisor $\Delta$ such that $K_{\mathcal{F}}+\Delta$ is $\mathbb{R}$-Cartier.

Note that if $(\mathcal{F}, \Delta)$ is foliated pair and $\Delta$ is a $\mathbb{Q}$-divisor, then $K_{\mathcal{F}}+\Delta$ is $\mathbb{Q}$-Cartier. Note also that we are typically interested only in the case when $\Delta \geq 0$, although it simplifies some computations to allow $\Delta$ to have negative coefficients.

Given a birational morphism $\pi: \widetilde{X} \rightarrow X$ and a foliated pair $(\mathcal{F}, \Delta)$ on $X$, let $\underset{\widetilde{\mathcal{F}}}{\widetilde{X}}$ be the pulled back foliation on $\tilde{X}$ and $\pi_{*}^{-1} \Delta$ be the strict transform of $\Delta$ in $\widetilde{X}$. We may write

$$
K_{\tilde{\mathcal{F}}}+\pi_{*}^{-1} \Delta=\pi^{*}\left(K_{\mathcal{F}}+\Delta\right)+\sum a(E, \mathcal{F}, \Delta) E,
$$

where $\pi_{*} K_{\widetilde{\mathcal{F}}}=K_{\mathcal{F}}$, the sum runs over all the prime $\pi$-exceptional divisors on $\widetilde{X}$ and the rational number $a(E, \mathcal{F}, \Delta)$ is called the discrepancy of $(\mathcal{F}, \Delta)$ with respect to $E$. If $\Delta=0$, then we will simply denote $a(E, \mathcal{F}, 0)$ by $a(E, \mathcal{F})$. Building on the work of McQuillan (e.g. see [31, Definition I.1.5]), we define:

Definition 2.2 Let $X$ be a normal variety and let $(\mathcal{F}, \Delta)$ be a foliated pair on $X$. We say that $(\mathcal{F}, \Delta)$ is terminal (resp. canonical, log terminal, log canonical) if $a(E, \mathcal{F}, \Delta)>0$ (resp. $\geq 0,>-\epsilon(E), \geq-\epsilon(E)$ ), for any birational morphism $\pi: \widetilde{X} \rightarrow X$ and for any prime $\pi$-exceptional divisor $E$ on $\widetilde{X}$.

We say that a foliation $\mathcal{F}$ is terminal (resp. canonical, $\log$ canonical) if the foliated pair $(\mathcal{F}, 0)$ is such.

We say that the foliated pair $(\mathcal{F}, \Delta)$ is klt if $\lfloor\Delta\rfloor=0$ and $a(E, \mathcal{F}, \Delta)>$ $-\epsilon(E)$ for any birational morphism $\pi: \tilde{X} \rightarrow X$ and for any $\pi$-exceptional prime divisor $E$ on $\tilde{X}$.

Let $P \in X$ be a, not necessarily closed, point of $X$. We say that the foliated pair $(\mathcal{F}, \Delta)$ is terminal (resp. canonical, log canonical) at $P$ if for any birational morphism $\pi: \widetilde{X} \rightarrow X$ and for any $\pi$-exceptional divisor $E$ on $\widetilde{X}$ whose centre in $X$ is the Zariski closure $\bar{P}$ of $P$, we have that the discrepancy of $E$ is $>0$ (resp. $\geq 0, \geq-\epsilon(E)$ ).

Notice that these notions are well defined, i.e., $\epsilon(E)$ and $a(E, \mathcal{F}, \Delta)$ are independent of $\pi$. 
Observe that in the case where $\mathcal{F}=T_{X}$, no exceptional divisor is invariant and so this definition recovers the usual definitions of $(\log )$ terminal, $(\log )$ canonical.

Remark 2.3 It follows from Definition 2.2 that if some component of Supp $\Delta$ is $\mathcal{F}$-invariant, then $(\mathcal{F}, \Delta)$ is not $\log$ canonical. Indeed, let $D$ be an $\mathcal{F}$-invariant component of $\Delta$ with coefficient $a>0$. Let $p \in D$ be a general point so that $X, D$ and $\mathcal{F}$ are all smooth at $p$. Let $b: X^{\prime} \rightarrow X$ be the blow up at $p$ and let $\mathcal{F}^{\prime}=b^{-1} \mathcal{F}$. Then $b$ extracts a single $\mathcal{F}^{\prime}$-invariant divisor $E$ of discrepancy

$$
a(E, \mathcal{F}, \Delta)=n-1-a
$$

where $n$ is the dimension of $X$. Let $D^{\prime}$ be the strict transform of $D$ and observe that $Z:=D^{\prime} \cap E$ is contained in Sing $\mathcal{F}^{\prime}$. Let $b^{\prime}: X^{\prime \prime} \rightarrow X^{\prime}$ be the blow up of $X^{\prime}$ in $Z$. Then $b^{\prime}$ extracts a divisor $E^{\prime}$ of discrepancy

$$
a\left(E^{\prime}, \mathcal{F}, \Delta\right)=n-2 a-1 \text {. }
$$

We may now blow up $E^{\prime} \cap D^{\prime \prime}$, where $D^{\prime \prime}$ is the strict transform of $D^{\prime}$ in $X^{\prime \prime}$, and, continuing this way, we produce a sequence of extractions of divisors with discrepancy $n-k a-1$ where $k=1,2, \ldots$. Thus, if $k$ is sufficiently large, then we extract a divisor $F$ such that

$$
a(F, \mathcal{F}, \Delta)<0=\epsilon(F)
$$

and, in particular, $(\mathcal{F}, \Delta)$ is not log canonical.

Definition 2.4 Given a foliated pair $(\mathcal{F}, \Delta)$, we say that $W \subset X$ is a log canonical centre (in short, lc centre) of $(\mathcal{F}, \Delta)$ provided $(\mathcal{F}, \Delta)$ is $\log$ canonical at the generic point of $W$ and there is some divisor $E$ of discrepancy $-\epsilon(E)$ on some model of $X$ dominating $W$.

Notice that in the case that $\epsilon(E)=0$ for all exceptional divisors $E$ over a centre the notions of log canonical and canonical coincide. In this case, we will still refer to canonical centres as log canonical centres.

We also remark that any $\mathcal{F}$-invariant divisor is an lc centre of $(\mathcal{F}, \Delta)$.

We have the following nice characterisation due to [31, Corollary I.2.2.]:

Proposition 2.5 Let $0 \in X$ be a normal surface germ with a terminal foliation $\mathcal{F}$ of rank one.

Then there exists a cyclic cover $\sigma: Y \rightarrow X$ such that $Y$ is a smooth surface and $\sigma^{-1} \mathcal{F}$ is a smooth foliation.

We also make note of the following easy fact: 
Lemma 2.6 Let $\pi: Y \rightarrow X$ be a proper birational morphism between normal varieties. Let $(\mathcal{F}, \Delta)$ be a foliated pair on $X$ and let $\mathcal{G}$ be the pulled back foliation of $\mathcal{F}$ on $Y$. Write $\pi^{*}\left(K_{\mathcal{F}}+\Delta\right)=K_{\mathcal{G}}+\Gamma$.

Then $a(E, \mathcal{F}, \Delta)=a(E, \mathcal{G}, \Gamma)$ for all $E$.

We will make frequent use of the following consequence of the negativity lemma:

Lemma 2.7 Let $\phi: X \rightarrow X^{\prime}$ be a birational map between normal varieties and let

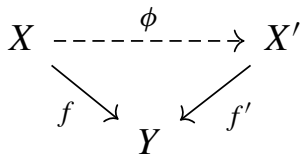

be a commutative diagram, where $Y$ is a normal variety and $f$ and $f^{\prime}$ are proper birational morphisms. Let $(\mathcal{F}, \Delta)$ be a foliated pair on $X$. Let $\mathcal{F}^{\prime}=$ $\phi_{*} \mathcal{F}$ and let $\Delta^{\prime}$ be a $\mathbb{Q}$-divisor on $X^{\prime}$ such that $f_{*} \Delta=f_{*}^{\prime} \Delta^{\prime}$. Assume that $-\left(K_{\mathcal{F}}+\Delta\right)$ is $f$-ample and $K_{\mathcal{F}^{\prime}}+\Delta^{\prime}$ is $f^{\prime}$-ample.

Then, for any valuation $E$ on $X$, we have

$$
a(E, \mathcal{F}, \Delta) \leq a\left(E, \mathcal{F}^{\prime}, \Delta^{\prime}\right) .
$$

Moreover, the inequality holds if $f$ or $f^{\prime}$ is not an isomorphism above the generic point of the centre of $E$ in $Y$.

Proof The proof is the same as [25, Lemma 3.38].

We now recall some facts from [10] on simple singularities. We say that the numbers $\lambda_{1}, \ldots, \lambda_{l} \in \mathbb{C}^{*}$ satisfy the non-resonant condition if for any non-negative integers $a_{1}, \ldots, a_{l}$ such that $\sum a_{i} \lambda_{i}=0$ we have that $a_{i}=0$ for all $i=1, \ldots, l$.

Definition 2.8 Let $\mathcal{F}$ be a co-rank one foliation on a smooth variety $X$ of dimension $n$. We say that $p \in X$ is a simple singularity for $\mathcal{F}$ provided that, in formal coordinates $x_{1}, \ldots, x_{n}$ around $p, N_{\mathcal{F}}^{*}$ is generated by a 1 -form which is in one of the following two forms, for some $1 \leq r \leq n$ :

1. There are $\lambda_{1}, \ldots, \lambda_{r} \in \mathbb{C}^{*}$, which satisfy the non-resonant condition and such that

$$
\omega=x_{1} \cdots x_{r} \cdot \sum_{i=1}^{r} \lambda_{i} \frac{d x_{i}}{x_{i}} .
$$


2. There is an integer $k \leq r$ such that

$$
\omega=x_{1} \cdots x_{r} \cdot\left(\sum_{i=1}^{k} p_{i} \frac{d x_{i}}{x_{i}}+\psi\left(x_{1}^{p_{1}} \cdots x_{k}^{p_{k}}\right) \sum_{i=2}^{r} \lambda_{i} \frac{d x_{i}}{x_{i}}\right),
$$

where $p_{1}, \ldots, p_{k}$ are positive integers without a common factor, $\psi(s)$ is a formal power series which is not a unit, and the numbers $\lambda_{2}, \ldots, \lambda_{r} \in \mathbb{C}^{*}$ satisfy the non-resonant condition.

The integer $r$ is called the dimension-type of the singularity. The $r$-uple $\left(\lambda_{1}, \ldots, \lambda_{r}\right)$ in (1) [resp. the $(r-1)$-uple $\left(\lambda_{2}, \ldots, \lambda_{r}\right)$ in (2)] is called the residual spectrum of the singularity (cf. [10, Remark 20]).

If $(X, D)$ is a normal crossing pair and $\mathcal{F}$ is a co-rank one foliation on $X$ then we say that $\mathcal{F}$ has simple singularities adapted to $D$ if $\mathcal{F}$ has simple singularities and, for every $p \in X$, we may choose formal coordinates around $p$ as above and such that the divisor $D \cup\left\{x_{1} \cdots x_{r}=0\right\}$ is also normal crossing at $p$ (cf. [10, Definitions 3, 13 and 14]).

A stratum of Sing $\mathcal{F}$ is a closed subvariety $Z \subset \operatorname{Sing} \mathcal{F}$ such that for all $p \in Z$ and coordinates $x_{1}, \ldots, x_{n}$ as above, in the formal neighbourhood of $X$ at $p$, we have that $Z$ is a stratum of $\left\{x_{1} \cdots x_{r}=0\right\}$.

By Cano [10, Main Theorem], every co-rank one foliation $\mathcal{F}$ on a smooth threefold $X$ admits a resolution $\pi: X^{\prime} \rightarrow X$ by blow ups centred in the singular locus of the foliation, such that the transformed foliation has simple singularities. By allowing blow ups in centres tangent to the foliation (but perhaps not contained in Sing $\mathcal{F}$ ) we may get that the transformed foliation has simple singularities adapted to Exc $\pi$. More generally, we may perform a sequence of blow ups so that the transform of $\mathcal{F}$ has simple singularities adapted to the transform of any divisor $D$ on $X$ (cf. [10, Theorem 3 and Sect. 4.5]).

We remark that if $\mathcal{F}$ is an algebraic foliation defined on an algebraic threefold and $D$ is an algebraic divisor then we only need to blow up in algebraic centres. Indeed, for centres contained in $\operatorname{Sing} \mathcal{F}$ this is obvious. If $Z$ is a centre which needs to be blown up and which is not contained in Sing $\mathcal{F}$ then $Z$ is either contained in the singular locus of $D$ or is contained in the tangency locus of $\mathcal{F}$ and $D$. Since $\mathcal{F}$ and $D$ are algebraic, these loci are also algebraic. Hence $Z$ is algebraic.

Lemma 2.9 Let $X$ be a smooth variety and let $\mathcal{F}$ be a co-rank one foliation with simple singularities on $X$.

Then $\mathcal{F}$ is canonical.

Proof Let $\pi: X^{\prime} \rightarrow X$ be a birational morphism, let $E \subset X^{\prime}$ be a divisor and let $Z=\pi(E)$. If $Z$ is not contained in Sing $\mathcal{F}$, then by shrinking about the 
generic point of $Z$ we may apply [1, Lemma 3.10] to conclude that $a(E, \mathcal{F}) \geq$ 0 .

Assume now that $Z \subset \operatorname{Sing} \mathcal{F}$. By shrinking about the generic point of $Z$, we may assume that $Z$ is smooth and, by Zariski's Lemma (cf. [25, Lemma 2.45]), after possibly replacing $X^{\prime}$ by a higher model, we may assume that $\pi$ is a composition of blow-ups of subvarieties centred on $Z$. By induction on the number of blow ups, it suffices to show that if $b: Y \rightarrow X$ is the blow up of $Z$ then

(i) if $E_{0}$ is the $b$-exceptional divisor then $a\left(E_{0}, \mathcal{F}\right) \geq 0$; and

(ii) $b^{-1} \mathcal{F}$ has simple singularities in a neighbourhood of $b^{-1}(z)$, where $z \in Z$ is a general point.

To prove (i), observe that if $W$ is a minimal stratum of Sing $\mathcal{F}$ containing $Z$ and $\omega$ is a 1-form defining $\mathcal{F}$ at a general point of $Z$, then $b^{*} \omega$ vanishes to order codim $W-1$ at the generic point of $E_{0}$. Thus,

$$
a\left(E_{0}, \mathcal{F}\right)=a\left(E_{0}, X\right)-(\operatorname{codim} W-1)=\operatorname{codim} Z-\operatorname{codim} W \geq 0
$$

and (i) follows.

We now prove (ii). Since we are only concerned about the behaviour at the general point of $Z$, we may assume without loss of generality that $Z$ is contained in a stratum of Sing $\mathcal{F}$ which meets no lower dimensional strata. Thus, If $p \in Z$ is a closed point then we may find formal coordinates $x_{1}, \ldots, x_{r}, y_{1}, \ldots, y_{n-r}$ around $p$, where $n$ is the dimension of $X, r$ is the dimension type of the singularity and if $\omega$ is a 1-form defining the foliation near $Z$ then $\omega$ is in one of the two forms in Definition 2.8 and

$$
Z=\left\{x_{1}=\cdots=x_{r}=y_{1}=\cdots=y_{l}=0\right\}
$$

for some $l \leq n-r$. Let $\omega^{\prime}:=\frac{1}{x_{1} \cdots x_{r}} \omega$.

Fix $i=1, \ldots, r$. Consider the chart for the blow up given by

$$
x_{1}=x_{i}^{\prime} x_{1}^{\prime} \ldots x_{i}=x_{i}^{\prime} \ldots x_{r}=x_{i}^{\prime} x_{r}^{\prime} \quad y_{1}=x_{i}^{\prime} y_{1}^{\prime} \ldots y_{s}=x_{i}^{\prime} y_{s}^{\prime} .
$$

If $\omega$ has a simple singularity of type (1) then

$$
\omega^{\prime}=\sum_{j=1}^{r} \lambda_{j} \frac{d x_{j}}{x_{j}}
$$

and, in this chart,

$$
b^{*} \omega^{\prime}=\sum_{j=1}^{r} \lambda_{j}^{\prime} \frac{d x_{j}^{\prime}}{x_{j}^{\prime}},
$$


where $\lambda_{j}^{\prime}=\lambda_{j}$ for $j \neq i$ and $\lambda_{i}^{\prime}=\sum_{j} \lambda_{j}$. In particular, notice that $\lambda_{1}^{\prime}, \ldots, \lambda_{r}^{\prime} \in \mathbb{C}^{*}$ satisfy the non-resonant condition, since it is a positive integral linear combination of a non-resonant spectrum, and so $b^{*} \omega^{\prime}$ is still a simple singularity of type (1). A similar computation holds in all other charts. Thus $b^{*} \omega^{\prime}$ defines a simple singularity of type (1).

So suppose that we have a simple singularity of type (2). Then

$$
\omega^{\prime}=\sum_{j=1}^{k} p_{j} \frac{d x_{j}}{x_{j}}+\psi\left(x_{1}^{p_{1}} \cdots x_{k}^{p_{k}}\right) \sum_{i=2}^{r} \lambda_{j} \frac{d x_{j}}{x_{j}} .
$$

Again, using coordinates for the blow up as above we see that if $1 \leq i \leq k$ then

$$
b^{*} \omega^{\prime}=\sum_{j=1}^{k} p_{j}^{\prime} \frac{d x_{j}^{\prime}}{x_{j}^{\prime}}+\psi\left(\left(x_{1}^{\prime}\right)^{p_{1}^{\prime}} \cdots\left(x_{k}^{\prime}\right)^{p_{k}^{\prime}}\right) \sum_{i=2}^{r} \lambda_{j}^{\prime} \frac{d x_{j}^{\prime}}{x_{j}^{\prime}}
$$

and if $i>k$ we have

$$
b^{*} \omega^{\prime}=\sum_{j=1}^{k} p_{j}^{\prime} \frac{d x_{j}^{\prime}}{x_{j}^{\prime}}+p_{i}^{\prime} \frac{d x_{i}^{\prime}}{x_{i}^{\prime}}+\psi\left(\left(x_{1}^{\prime}\right)^{p_{1}^{\prime}} \ldots\left(x_{k}^{\prime}\right)^{p_{k}^{\prime}}\left(x_{i}^{\prime}\right)^{p_{i}^{\prime}}\right) \sum_{i=2}^{r} \lambda_{j}^{\prime} \frac{d x_{j}^{\prime}}{x_{j}^{\prime}},
$$

where $p_{j}^{\prime}=p_{j}$ if $j \neq i$ and $p_{i}^{\prime}=p_{1}+\cdots+p_{k}$ and $\lambda_{j}^{\prime}$ is defined as above. Again, it follows that $\lambda_{2}^{\prime}, \ldots, \lambda_{r}^{\prime}$ satisfy the non-resonant condition and, similarly as above, $b^{*} \omega$ defines a simple singularity of type (2).

We remark that in both these cases the exceptional divisor of the blow up is invariant.

Thus, $b^{-1} \mathcal{F}$ has simple singularities in a neighbourhood of $b^{-1}(z)$ and (ii) follows.

The converse of this statement is false (e.g. see [41, Example 2.16]).

Definition 2.10 Given a normal variety $X$ and a foliation $\mathcal{F}$ on $X$, we say that $\mathcal{F}$ has non-dicritical singularities if for any closed point $q \in X$ and any proper birational morphism $\pi: X^{\prime} \rightarrow X$ such that $\pi^{-1}(q)$ is a divisor we have that each component of $\pi^{-1}(q)$ is invariant by $\pi^{-1} \mathcal{F}$.

Example 2.11 Let $\lambda \in \mathbb{R}$. Consider the rank one foliation $\mathcal{F}_{\lambda}$ on $\mathbb{C}^{2}$ generated by $x \partial_{x}+\lambda y \partial_{y}$. For $\lambda \in \mathbb{Q}_{>0}$ we can see that $\mathcal{F}_{\lambda}$ is dicritical, and otherwise is non-dicrtical. See [9, pg. 7] for an explicit resolution of $\mathcal{F}_{\lambda}$ when $\lambda \in \mathbb{Q}_{>0}$.

Definition 2.12 Let $X$ be a normal variety and let $\mathcal{F}$ be a co-rank one foliation with non-dicritical singularities. 
We say that a subvariety $W \subset X$ (possibly contained in Sing $X$ or Sing $\mathcal{F}$ ) is tangent to $\mathcal{F}$ if for any birational morphism $\pi: X^{\prime} \rightarrow X$ and any divisor $E$ on $X$ such that $E$ dominates $W$, we have that $E$ is $\mathcal{F}^{\prime}$-invariant, where $\mathcal{F}^{\prime}$ is the pulled back foliation on $X^{\prime}$. Otherwise, we say that $W \subset X$ is transverse to $\mathcal{F}$.

Note that the definitions above differ slightly from the usual ones, but, in our opinion, they are more flexible when working with singular varieties. In particular, if $W \subset X$ is a subvariety which is not contained in Sing $X \cup \operatorname{Sing} \mathcal{F}$, then our definition of tangency coincides with the classic one. Note also that for divisors the notions of tangency and invariance coincide.

Assume now that $X$ is a normal variety, $\mathcal{F}$ is a co-rank one foliation on $X$ and $W \subset X$ is an irreducible subvariety which is not contained in Sing $X \cup \operatorname{Sing} \mathcal{F}$ and which is transverse to $\mathcal{F}$. Let $v: W^{v} \rightarrow W$ be its normalisation. Let $U \subset X$ be a proper open subset which intersect $W$ and which is contained in the smooth locus of $X$ and let $V=v^{-1}(U \cap W)$. Then the composition of the natural maps

$$
\left.\left.\mathcal{N}_{\mathcal{F}}^{*}\right|_{V} \rightarrow \Omega_{X}^{1}\right|_{V} \rightarrow \Omega_{V}^{1}
$$

induces a co-rank one foliation $\mathcal{G}_{V}$ on $V$ which extends naturally to a foliation $\mathcal{G}$ on $W^{v}$, called the restricted foliation of $\mathcal{F}$ on $W^{\nu}$.

If $X$ is a smooth variety and $\mathcal{F}$ is a co-rank one foliation on $X$ then we say that $\mathcal{F}$ is strongly non-dicritical if for any sequence of blow ups

$$
X_{n} \stackrel{p_{n}}{\longrightarrow} \cdots X_{1} \stackrel{p_{1}}{\longrightarrow} X
$$

in smooth centres tangent to $\mathcal{F}_{i}$ or smooth centres contained in Sing $\mathcal{F}_{i}$, where $\mathcal{F}_{i}$ is the transformed foliation on $X_{i}$, we have that the exceptional locus of $X_{n} \rightarrow X$ is $\mathcal{F}_{n}$-invariant (cf. [12]). We remark that we allow these maps to be blow ups along analytic subvarieties.

Remark 2.13 Simple singularities are strongly non-dicritical. Indeed, by [12, Théorème 4] non-dicriticality can be checked by blowing up in permissible centres (cf. [10, Definition 1]). However, as we saw in the proof of Lemma 2.9 the exceptional divisor for such a blow up is always invariant by the induced foliation.

In [12, Théorème 4], the following characterisation of strong nondicriticality is given. A germ of a co-rank one foliation $\mathcal{F}$ on $0 \in \mathbb{C}^{n}$ is strongly non-dicritical if and only if there does not exists a germ of a surface $0 \in Z \subset \mathbb{C}^{n}$ such that $Z$ is transverse to $\mathcal{F}$ and such that the restricted foliation to $Z$ admits infinitely many invariant curves passing through Sing $\mathcal{F}$. 
We now show that, in the case of smooth threefolds, all these notions of non-dicriticality coincide:

Lemma 2.14 Let $X$ be a smooth threefold and let $\mathcal{F}$ be a co-rank one foliation on $X$.

Then $\mathcal{F}$ is non-dicritical if and only if it is strongly non-dicritical.

Proof Suppose that $\mathcal{F}$ is strongly non-dicritical. Let $\pi: X^{\prime} \rightarrow X$ be a proper birational morphism, let $q \in X$ be a closed point and suppose that $E \subset$ $\pi^{-1}(q)$ is a prime divisor. Suppose for sake of contradiction that $E$ is not $\pi^{-1} \mathcal{F}$-invariant. Let $H \subset X^{\prime}$ be a general hypersurface such that if $\mathcal{G}$ is the foliation restricted to $H$ then $E \cap H$ is transverse to $\mathcal{G}$. Then, through a general point $P \in E \cap H$ there is a germ of a $\mathcal{G}$-invariant curve passing through $P$, call it $\Sigma_{P}$. It follows that if $Z=\pi(H)$ then $Z$ is transverse to $\mathcal{F}$ and the restricted foliation admits infinitely many invariant curves passing through $q=\pi(E \cap H)$, namely, $\pi\left(\Sigma_{P}\right)$ as we let $P$ vary over points of $E \cap H$, a contradiction. In particular, $q \in \operatorname{Sing} \mathcal{F}$. Thus, $\mathcal{F}$ is non-dicritical.

Now suppose that $\mathcal{F}$ is non-dicritical. Let $\pi: X^{\prime} \rightarrow X$ be a resolution of singularities so that $\left(X^{\prime}\right.$, Exc $\left.\pi\right)$ is $\log$ smooth and $\mathcal{F}^{\prime}:=\pi^{-1} \mathcal{F}$ has simple singularities adapted to Exc $\pi$ (cf. Definition 2.8). In particular, if $E$ is a component of Exc $\pi$ which is not $\mathcal{F}^{\prime}$-invariant then Sing $\mathcal{F}^{\prime} \cap E$ has no one-dimensional components. We may also take $\pi$ to be a sequence of blow ups centred either in Sing $\mathcal{F}$ or centres tangent to the foliation. Since, by Remark 2.13, simple singularities are strongly non-dicritical, it suffices to show that $\operatorname{Exc} \pi$ is $\mathcal{F}^{\prime}$-invariant.

If $E$ is a component of Exc $\pi$ such that $\pi(E)$ is zero-dimensional or $\pi(E)$ is not contained in Sing $\mathcal{F}$ then it follows immediately that $E$ is $\mathcal{F}^{\prime}$-invariant. So suppose that $\pi(E)$ is a curve contained in Sing $\mathcal{F}$. Since $\mathcal{F}$ is non-dicritical it follows that the fibres of $E \rightarrow \pi(E)$ are tangent to $\mathcal{F}^{\prime}$. Suppose for sake of contradiction that $E$ is not $\mathcal{F}^{\prime}$-invariant.

If $\mathcal{G}$ is the foliation restricted to $E$ then $\mathcal{G}$ is the foliation induced by $E \rightarrow \pi(E)$ and is therefore smooth over the generic point of $\pi(E)$. Since Sing $\mathcal{F}^{\prime} \cap E$ has no one-dimensional components, it follows that $\mathcal{F}^{\prime}$ is smooth in a neighbourhood of a general fibre of $E \rightarrow \pi(E)$. Next, we claim that if $E^{\prime}$ is any other $\pi$-exceptional divisor dominating $\pi(E)$ then $E^{\prime}$ is not $\mathcal{F}^{\prime}$-invariant. Indeed, suppose otherwise. Without loss of generality we may assume that $E^{\prime} \cap E \neq \emptyset$ and $E \cap E^{\prime}$ dominates $\pi(E)$. However $E \cap E^{\prime}$ is $\mathcal{G}$-invariant since $E^{\prime}$ is $\mathcal{F}^{\prime}$-invariant and so $E \cap E^{\prime}$ is contained in a fibre of $E \rightarrow \pi(E)$, a contradiction.

Shrinking about a neighborhood of the general point of $\pi(E)$ we may therefore assume that every $\pi$-exceptional divisor is transverse to $\mathcal{F}^{\prime}$ and that $\mathcal{F}^{\prime}$ is smooth. 
Now, let $\omega$ be a 1-form defining $\mathcal{F}$ in a neighborhood of a general point of $\pi(E)$. Since $\pi(E)$ is contained in Sing $\mathcal{F}$ it follows that $\pi^{*} \omega$ must vanish along Exc $\pi$ and, in particular, it follows that that $N_{\mathcal{F}^{\prime}}^{*}=F$ where $F \geq 0$ is $\pi$-excpetional and $F \neq 0$.

On the other hand, it is easy to verify that $K_{\mathcal{F}^{\prime}}$ and $K_{X^{\prime}}$ are numerically equivalent over $X$. Indeed, one can check (as in the proof of [41, Lemma 3.11]) that if $E^{\prime}$ is any $\pi$-exceptional divisor dominating $\pi(E)$ and if $C$ is a general fibre of $E^{\prime} \rightarrow \pi(E)$ then $\left(K_{\mathcal{F}^{\prime}}+E\right) \cdot=\left(K_{X^{\prime}}+E^{\prime}\right) \cdot C=-2$ and so $K_{\mathcal{F}^{\prime}} \cdot C=K_{X^{\prime}} \cdot C$. This, together with the equality $K_{X^{\prime}}=K_{\mathcal{F}^{\prime}}+N_{\mathcal{F}^{\prime}}^{*}$ implies that $N_{\mathcal{F}^{\prime}}^{*}=F$ is numerically trivial over $X$. Since $F \neq 0$, the negativity lemma (e.g. see [25, Lemma 3.39]) gives us a contradiction.

Remark 2.15 The above proof shows that if $X$ is a smooth threefold, $\mathcal{F}$ is a non-dicritical co-rank one foliation on $X, \pi: X^{\prime} \rightarrow X$ is a birational morphism and $W$ is $\mathcal{F}$-invariant, then $\pi^{-1}(W)$ is $\mathcal{F}^{\prime}$-invariant where $\mathcal{F}^{\prime}$ is the transformed foliation on $X^{\prime}$.

Remark 2.16 Let $X$ be a normal threefold and let $\mathcal{F}$ be a co-rank one foliation with non-dicritical singularities. Let $\pi: X^{\prime} \rightarrow X$ be a birational morphism and let $\mathcal{F}^{\prime}$ be the transformed foliation on $X^{\prime}$. Assume that there exists a prime $\pi$-exceptional divisor $E$, which is $\mathcal{F}^{\prime}$-invariant and whose centre in $X$ is $W$. Then every $\pi$-exceptional divisor $E$ whose centre in $X$ is $W$ is $\mathcal{F}^{\prime}$-invariant. Indeed, the proof of this fact follows exactly as in the proof of Lemma 2.14.

Definition 2.17 Given a normal germ $0 \in X$ (resp. the formal completion $0 \in \widehat{X}$ of a normal variety $X$ at the point $0 \in X$ ) with a co-rank one foliation $\mathcal{F}$ such that 0 is a singular point for $\mathcal{F}$, we call an irreducible hypersurface germ (resp. a formal hypersurface) $0 \in S$ a separatrix (resp. formal separatrix) if it is $\mathcal{F}$-invariant.

Let $0 \in X$ be a smooth germ and let $\mathcal{F}$ be a co-rank one foliation $\mathcal{F}$ on $X$ defined by a 1-form $\omega$. Sometimes, in the literature, a formal separatrix is defined to be an irreducible and reduced formal power series $f$ such that $f$ divides $d f \wedge \omega$. We claim that, under this assumption, $\{f=0\}$ defines a formal hypersurface which is $\mathcal{F}$-invariant. Indeed, let $v$ be a vector field such that $v(\omega)=0$. On one hand, $v(d f \wedge \omega)$ is necessarily divisble by $f$ since $d f \wedge \omega$ is. On the other hand, we can compute $v(d f \wedge \omega)=v(d f) \omega-d f v(\omega)=v(d f) \omega$ which implies that $v(d f)$ is divisible by $f$, i.e., the ideal $(f)$ is invariant by $v$, as required. Conversely, if $\{f=0\}$ is $\mathcal{F}$-invariant then $d f \wedge \omega$ is divisible by $f$. Indeed, after replacing $(X,\{f=0\})$ by its log resolution whose existence is guaranteed by [42, Theorems 1.1.9 and 1.1.13], we may assume that the pair $(X,\{f=0\})$ is log smooth. Thus, we may assume that in some formal coordinates $x_{1}, \ldots, x_{n}$ we may write $f=x_{1}$. Now write $\omega=\sum a_{i} d x_{i}$ for some functions $a_{1}, \ldots, a_{n}$ and suppose for sake of contradiction that there 
exists $j \neq 1$ such that $a_{j} \notin\left(x_{1}\right)$. In this case $\partial:=a_{j} \frac{\partial}{\partial x_{1}}-a_{1} \frac{\partial}{\partial x_{j}}$ is a local vector field in $\mathcal{F}$. However, $\partial\left(x_{1}\right)=a_{j} \notin\left(x_{1}\right)$ and so $\left\{x_{1}=0\right\}$ is not invariant, a contradiction.

Example 2.18 Let $\mathcal{F}$ be a co-rank one foliation on a smooth variety $X$ with a simple singularity at $p \in X$ of dimension type $r$. Let $x_{1}, \ldots, x_{n}$ be formal coordinates as in Definition 2.8. Then $\left\{x_{i}=0\right\}$ is a formal separatrix for each $i=1, \ldots, r$ and moreover these are the only formal separatrices at $p$, see $[10$, Appendix: About simple singularities].

Note that away from the singular locus of $\mathcal{F}$ a separatrix is in fact a leaf. Furthermore being non-dicritical implies that there are only finitely many separatrices through a singular point. The converse of this statement is false.

Lemma 2.19 Let $X$ be a smooth variety of dimension $n$ and let $\mathcal{F}$ be a co-rank one foliation on $X$ with simple singularities. Suppose that $S \subset \operatorname{Sing} \mathcal{F}$ is a subvariety such that $\operatorname{dim} S \geq 1$. Let $H \subset X$ be a general element of a base point free linear system and let $\mathcal{G}$ be the restricted foliation.

Then $\mathcal{G}$ has simple singularities at a general point of $S \cap H$.

Proof Remark 2.13 implies that $\mathcal{F}$ admits non-dicritical singularities. Let $P \in$ $S$ be a general closed point and let $r \leq n-1$ be the dimension type of $\mathcal{F}$ at $P$. Note that this condition is generic in Sing $\mathcal{F}$.

Let $D_{1}, \ldots, D_{r}$ be the separatrices of $\mathcal{F}$ at $P$, including the formal ones. Let $H$ be a hyperplane passing through $P$ such that $\left(\widehat{X}, D_{1}+\cdots+D_{r}+H\right)$ is a normal crossings pair where $\widehat{X}$ is the formal completion of $X$ at $P$. If $\omega$ is a 1 -form defining $\mathcal{F}$ it is easy to check that $\omega$ restricted to $H$ is still a 1 -form which is of one of the types listed in Definition 2.8. Thus, the restricted foliation will have simple singularities near $P$. It follows from [10, Proposition 14] that having pre-simple singularities is an open condition in Sing $\mathcal{F}$, and the proof there works just as well to imply that having simple singularities is an open condition in Sing $\mathcal{F}$.

Even for simple foliation singularities it is possible that there are separatrices which do not converge. However, as the following definition/result shows there is always at least one convergent separatrix along a simple foliation singularity of codimension two.

Lemma 2.20 For a simple singularity of type (1), all separatrices are convergent.

For a simple singularity of type (2), around a general point of a codimension two component of the the singular locus we can write $\omega=$ pydx $+q x d y+$ $x \psi\left(x^{p} y^{q}\right) \lambda d y$. The hypersurface $\{x=0\}$ is a convergent separatrix, called the strong separatrix.

Proof This is proven in [11, Part II Sect. 5]. 


\subsection{Foliation with $K_{\mathcal{F}}$ not pseudo-effective}

We make note of the following easy consequence of [14].

Lemma 2.21 Let $X$ be a normal variety and let $\mathcal{F}$ be a foliation on $X$ such that $K_{\mathcal{F}}$ is $\mathbb{Q}$-Cartier and $K_{\mathcal{F}}$ is not pseudo-effective. Then $\mathcal{F}$ is uniruled, i.e., there exists a family of rational curves covering $X$ and tangent to $\mathcal{F}$.

Proof Let $\pi: X^{\prime} \rightarrow X$ be a resolution of singularities and let $\mathcal{F}^{\prime}$ be the pulled back foliation. Then observe that $K_{\mathcal{F}^{\prime}}$ is not pseudo-effective and so we may freely assume that $X$ is smooth.

Let $\alpha$ be a movable class so that $K_{\mathcal{F}} \cdot \alpha<0$. Let $\mathcal{E} \subset \mathcal{F}$ be a maximal destabilizing subsheaf of $\mathcal{F}$ with respect to $\alpha$. It follows that $\mathcal{E}$ defines a foliation with $\mu_{\alpha, \min }(\mathcal{E})>0$ and so by [14, Theorem 1.1] $\mathcal{E}$ is a foliation with rationally connected leaves, from which our claim follows.

\subsection{Steps of the minimal model program}

We recall some of the main definitions commonly used in the Minimal Model Program. Let $X$ be a normal projective variety. We denote by $N_{1}(X)$ the $\mathbb{R}$ vector space spanned by 1-cycles on $X$ modulo numerical equivalence (e.g. see [25, Definition 1.16]). We denote by $N E(X) \subset N_{1}(X)$ the subset of effective 1 -cycles $\left[\sum_{i=1}^{k} a_{i} C_{i}\right.$ ] where $a_{1}, \ldots, a_{k}$ are positive real numbers and $C_{1}, \ldots, C_{k}$ are curves in $X$, and we denote by $\overline{N E(X)}$ its closure (e.g. see [25, Definition 1.17]). A ray is a one-dimensional subcone $R$ of $\overline{N E(X)}$ and it is called extremal if for any $u, v \in \overline{N E(X)}$ such that $u+v \in R$, we have that $u, v \in R$. If $D$ is a $\mathbb{Q}$-Cartier $\mathbb{Q}$-divisor on $X$ then the extremal ray $R$ is said to be $D$-negative if $D \cdot C<0$ for any curve $C$ such that $[C] \in$ $R$. Given an extremal ray $R \subset \overline{N E}(X)$, we define the locus of $R$, denoted $\operatorname{loc}(R)$, to be the set of all those points $x \in X$ such that there exists a curve $C$ with $x \in C$ and $[C] \in R$. A projective birational morphism $f: X \rightarrow Y$ between normal projective varieties is said to be a flipping contraction, or small contraction, if its exceptional locus has codimension at least two and it is called a divisorial contraction if its exceptional locus is a divisor. Moreover, the birational morphism $f: X \rightarrow Y$ is said to be an extremal contraction if the relative Picard number $\rho(X / Y):=\rho(X)-\rho(Y)$ is equal to one. Given an extremal ray $R \subset \overline{N E}(X)$, an extremal contraction $f: X \rightarrow Y$ is said to be associated to $R$ if the locus of $R$ coincides with the exceptional locus of $f$.

Let $D$ be a $\mathbb{Q}$-Cartier $\mathbb{Q}$-divisor on a normal projective variety $X$, let $R$ be a $D$-negative extremal ray and let $f: X \rightarrow Y$ be a flipping contraction associated to $R$. Note, in particular, that $-D$ is $f$-ample. Then the $D$-flip is a birational map $\phi: X \rightarrow X^{+}$such that

1. $\phi$ is an isomorphism in codimension one, 
2. there exists an extremal small contraction $f^{+}: X^{+} \rightarrow Y$, and

3. $D^{+}:=\phi_{*} D$ is $f^{+}$-ample.

Note that the $D$-flip, if it exists, is unique.

We refer to [7, Definition 3.6.4] for the definition of ample models and we observe that if $\phi: X \rightarrow X^{+}$is a $D$-flip with flipping contraction $f: X \rightarrow Y$ then $X^{+}$is the ample model of $D$ over $Y$.

Let $\phi: X \rightarrow Y$ be a birational contraction between normal projective varieties and let $D$ be a $\mathbb{Q}$-Cartier $\mathbb{Q}$-divisor on $X$. Then $\phi$ is said to be a step of a $D$-MMP if it is either a $D$-flip or a divisorial contraction associated to a $D$-negative extremal ray. More in general, $\phi$ is said to be a sequence of steps of a $D$-MMP if we can decompose $\phi$ as

$$
X=X_{0} \rightarrow X_{1} \cdots \rightarrow X_{\ell}=Y
$$

so that the birational contraction $X_{i} \rightarrow X_{i+1}$ is a step of a $D_{i}$-MMP where $D_{i}$ is the strict transform of $D$ on $X_{i}$. Note that if $X$ is $\mathbb{Q}$-factorial, then $Y$ is also $\mathbb{Q}$-factorial.

If we replace the projective variety $X$ by a projective morphism $\pi: X \rightarrow U$ between normal varieties, then all the definitions above admit a relative version, by replacing each variety by one admitting a projective morphism to $U$, and each birational map by a birational map over $U$, in a similar fashion as in the classical MMP (e.g. see [25, Sect. 3.6]).

\subsection{A result from the classical MMP}

We will need to make use of some techniques from the classical MMP.

Definition 2.22 Let $X$ be a normal variety. We say that a birational morphism $f: Y \rightarrow X$ is a small $\mathbb{Q}$-factorialisation if the following holds:

1. $f$ is an isomorphism in codimension one,

2. $f$ is a projective morphism, and

3. $Y$ is $\mathbb{Q}$-factorial.

Theorem 2.23 [7, Corollary 1.4.3] Let $X$ be a normal algebraic variety of dimension three and let $D \geq 0$ be such that $(X, D)$ is klt.

Then there exists a small $\mathbb{Q}$-factorialisation for $X$. Moreover, if we write $K_{Y}+D_{Y}=f^{*}\left(K_{X}+D\right)$ then $\left(Y, D_{Y}\right)$ is klt.

Remark 2.24 Since flips are known to exist over complex analytic varieties (cf. [39, Main Theorem]), the same proof as in [7] implies the existence of a small $\mathbb{Q}$-factorialisation for a klt pair $(X, D)$, where $X$ is a complex analytic space of dimension three. 


\section{F-dlt foliated pairs and basic adjunction type results}

The goal of this section is to define a new category of foliated log pair singularities, namely F-dlt pairs. These are analogous of dlt log pairs in the classical MMP and they seem to be the most suitable singularities to run a foliated MMP. In particular, we prove several properties satisfied by these pairs, which we use later on in the paper.

\subsection{Foliated log smooth pairs}

Definition 3.1 Given a normal variety $X$, a co-rank one foliation $\mathcal{F}$ and a foliated pair $(\mathcal{F}, \Delta)$ we say that $(\mathcal{F}, \Delta)$ is foliated log smooth provided the following hold:

1. $(X, \Delta)$ is $\log$ smooth,

2. $\mathcal{F}$ has simple singularities, and

3. if $S$ is the support of the non $\mathcal{F}$-invariant components of $\Delta, p \in S$ is a closed point and $\Sigma_{1}, \ldots, \Sigma_{k}$ are the (possibly formal) $\mathcal{F}$-invariant divisors passing through $p$, then $S \cup \Sigma_{1} \cup \cdots \cup \Sigma_{k}$ is a normal crossings divisor at p.

Given a normal variety $X$, a co-rank one foliation $\mathcal{F}$ and a foliated pair $(\mathcal{F}, \Delta)$, a foliated $\log$ resolution, or in short $\log$ resolution, is a proper birational morphism $\pi: Y \rightarrow X$ so that Exc $\pi$ is a divisor and $\left(\mathcal{G}, \pi_{*}^{-1} \Delta+\sum E\right)$ is foliated $\log$ smooth where $\mathcal{G}$ is the pulled back foliation on $Y$ and the sum runs over all the $\pi$-exceptional divisors.

Remark 3.2 - If $X$ is a surface, then the existence of a foliated log resolution follows from a result of Seidenberg [38]. If $X$ is a threefold, then such a resolution exists by [10].

- Items (2) and (3) in Definition 3.1 imply that each component of $S$ is generically transverse to the foliation, no strata of $S$ is tangent to the foliation and no strata of Sing $\mathcal{F}$ is contained in $S$.

- If $\mathcal{F}$ is $\log$ smooth and if $D$ is a $\mathcal{F}$-invariant divisor then it is not necessarily the case that $D$ is smooth, although it will have at worst normal crossings singularities.

Lemma 3.3 Let $(\mathcal{F}, \Delta)$ be a foliated log smooth pair on a variety $X$, where $\Delta=\sum a_{i} D_{i}$ is $a \mathbb{Q}$-divisor such that $0 \leq a_{i} \leq 1$ and $D_{i}$ is not $\mathcal{F}$-invariant for every $i$.

Then $(\mathcal{F}, \Delta)$ is log canonical.

Proof By Lemma 2.9, since $\mathcal{F}$ has simple singularities, it follows that $\mathcal{F}$ is canonical. 
Now let $\pi: Y \rightarrow X$ be a blow up of subvariety $Z \subset$ Supp $\Delta$ where $Z$ has codimension $k$. Let $E$ be the exceptional divisor. We compute the discrepancy of this blow up as follows:

1. If $Z$ is transverse to the foliation then the discrepancy is

$$
(k-1)-\sum_{\left\{i \mid Z \subset D_{i}\right\}} a_{i} \geq-1=-\epsilon(E),
$$

where the inequality holds since $k \geq \#\left\{i \mid Z \subset D_{i}\right\}$ by Item (1) in Definition 3.1.

2. If $Z$ is tangent to the foliation but not contained in Sing $\mathcal{F}$ then the discrepancy is

$$
(k-1)-\sum_{\left\{i \mid Z \subset D_{i}\right\}} a_{i} \geq 0=-\epsilon(E),
$$

where the inequality holds since $k \geq \#\left\{i \mid Z \subset D_{i}\right\}+1$ by Item (3) in Definition 3.1.

3. If $Z \subset$ Sing $\mathcal{F}$ then let $m$ be the codimension of the minimal strata of Sing $\mathcal{F}$ containing $Z$. The discrepancy of the blow up is

$$
(k-1)-(m-1)-\sum_{\left\{i \mid Z \subset D_{i}\right\}} a_{i} \geq 0=-\epsilon(E),
$$

where the inequality holds since $k \geq m+\#\left\{i \mid Z \subset D_{i}\right\}$ by Item (3) in Definition 3.1.

As in the proof of Lemma 2.9, it follows that if $\mathcal{G}$ is the transformed foliation on $Y$ and $\Delta^{\prime}$ is the strict transform of $\Delta$ in $Y$, then $\left(\mathcal{G}, \Delta^{\prime}+E\right)$ is foliated $\log$ smooth. Thus, the result then follows by induction.

Note that in contrast to the classical situation, if $(\mathcal{F}, \Delta)$ is a foliated $\log$ smooth pair then $(\mathcal{F}, \Delta)$ may have infinitely many lc centres:

Example 3.4 Let $\left(\mathcal{F}, D_{1}+D_{2}\right)$ be a foliated log smooth pair on a threefold $X$, for some prime divisors $D_{1}$ and $D_{2}$ which are not $\mathcal{F}$-invariant. Suppose that $Z=D_{1} \cap D_{2}$ is non-empty, disjoint from Sing $\mathcal{F}$ and that $Z$ is transverse to $\mathcal{F}$. Then $Z$ is an lc centre of $\left(\mathcal{F}, D_{1}+D_{2}\right)$. Moreover, if $p \in Z$ and if $\pi: Y \rightarrow X$ is the blow up at $p$ with exceptional divisor $E$ then the discrepancy with respect to $E$ is $0=\epsilon(E)$ and so $p$ is an lc centre of $\left(\mathcal{F}, D_{1}+D_{2}\right)$. In particular, $\left(\mathcal{F}, D_{1}+D_{2}\right)$ admits infinitely many lc centres.

Note also that if $\mathcal{F}$ is a foliation on a smooth projective variety $X$ which is induced by a fibration onto a curve then any smooth vertical fibre is an lc centre. 


\subsection{Extending separatrices}

We provide a general result on the existence of formal separatrices which is a slight generalisation of the results in [11, Sect. IV].

Lemma 3.5 Let $X$ be a normal quasi-projective threefold. Let $\mathcal{F}$ be a corank one foliation on $X$ with non-dicritical singularities. Let $V \subset X$ be a subvariety tangent to $\mathcal{F}$, let $q \in V$ be any point and let $\widehat{S}_{q}$ be a, possibly formal, $\mathcal{F}$-invariant divisor containing $q$. Let $\widehat{X}$ be the formal completion of $X$ along $V$.

Then there exists an $\widehat{\mathcal{F}}$-invariant formal subscheme $\widehat{S}$ on $\widehat{X}$ which contains $\widehat{S}_{q}$ near $q$.

Moreover, if $\widehat{S}_{q}$ is in fact convergent, then we may take $\widehat{S}$ to be convergent.

Proof Let $\pi: W \rightarrow X$ be a high enough foliated $\log$ resolution so that $\pi^{-1}(V)=E$ is a divisor. By definition, we see that $E$ is $\pi^{-1} \mathcal{F}$-invariant. Let $\widehat{W}$ be the completion of $W$ along $E$ and let $\hat{\pi}: \widehat{W} \rightarrow \widehat{X}$ be the induced morphism.

Let $\widehat{X}_{/ q}$ denote the formal completion of $X$ along $q$ and let $\widehat{W}_{/ \pi^{-1}(q)}$ denote the formal completion of $W$ along $\pi^{-1}(q)$. Note that we have morphisms $\widehat{X}_{/ q} \rightarrow \widehat{X}$ and $\widehat{W}_{/ \pi^{-1}(q)} \rightarrow \widehat{W}$ which commute with the induced morphism $\widehat{W}_{/ \pi^{-1}(q)} \rightarrow \widehat{X}_{/ q}$ and $\widehat{W} \rightarrow \widehat{X}$. Since $\widehat{S}_{q}$ is a formal subscheme of $\widehat{X}_{/ q}$ we may take its strict transform on $\widehat{W}_{/ \pi^{-1}(q)}$, call it $\widehat{S}_{q}^{\prime}$. Recall that we can construct the strict transform as follows: let $\widehat{X}_{/ q}=\operatorname{Spf} A, \widehat{S}_{q}=\operatorname{Spf} B$ and let $\pi$ be given by the blow up along an ideal $I$. Let $\tilde{W}$ be the blow up of Spec $A$ along $I \otimes A$. If $\tilde{S}=\operatorname{Spec} B$ we may define the strict transform $\tilde{S}^{\prime}$ as the blow up of $\tilde{S}$ along the ideal $I \otimes_{A} B,\left[23\right.$, Corollary II.7.15]. We may take $\widehat{S}_{q}^{\prime}$ to be the formal completion of $\tilde{S}^{\prime}$ along $\tilde{S}^{\prime} \cap \pi^{-1}(q)$.

The arguments in [11, Sect. IV] and their slight extension in [41, Lemma 5.3] show that if $\widehat{S}_{q}^{\prime}$ is convergent then we can extend $\widehat{S}_{q}^{\prime}$ to a $\pi^{-1} \mathcal{F}$-invariant formal subscheme $\widehat{S}^{\prime}$ of $\widehat{W}$. In fact, the arguments given in [41] work even if $\widehat{S}_{q}^{\prime}$ is not convergent as in the proof of [11, Theorem IV.2.1].

For the reader's convenience we briefly indicate some of the important ideas of the proof of [11, Theorem IV.2.1]. Let $\omega$ be a 1-form defining a simple singularity on $0 \in U \subset \mathbb{C}^{3}$ with coordinates $(x, y, z)$. Suppose that the dimension type of $\omega$ is 3 (the case where the dimension type is 2 can be handled in a similar manner). By the work of [11, Sect. 2] we know that two of the separatrices at 0 are convergent. So, after performing a holomorphic change of coordinates we may assume that they are given by $\{x=0\}$ and $\{y=0\}$. It follows that the formal separatrix at 0 may be defined by an equation $f=z+$ $\phi(x, y)$ where $\phi(x, y) \in(x y) \mathbb{C}[[x, y]]$. By [11, Proposition II.5.4] it follows that there exists a bidisc $V \subset \mathbb{C}^{2}$ such that in fact $\phi(x, y)=\sum \phi_{i}(x, y)(x y)^{i}$ 
where $\phi_{i}(x, y) \in \mathcal{O}_{\mathbb{C}^{2}}(V)$. In particular, up to shrinking $U$, if $\widehat{U}$ denotes the formal completion of $U$ along $\{x y=0\}$ we see that in fact $z+\phi(x, y) \in$ $H^{0}\left(\widehat{U}, \mathcal{O}_{\widehat{U}}\right)$. In particular, we have extended the formal separatrix, a priori only defined on the formal completion of $U$ at 0 , to the formal completion of $U$ along the union of the convergent separatrices. Using this local extension result we see that the arguments given for extending convergent separatrices work for extending formal separatrices.

Let $\mathcal{I}_{\widehat{S}^{\prime}} \subset \mathcal{O}_{\widehat{W}}$ be the ideal sheaf corresponding to $\widehat{S}^{\prime}$. By the proper mapping theorem for formal schemes [22, Théorème 3.4.2], $\hat{\pi}_{*} I_{\widehat{S}^{\prime}}$ is a coherent sheaf, and since $\hat{\pi}_{*} \mathcal{O}_{\widehat{W}}=\mathcal{O}_{\widehat{X}}$ we see that it is in fact an ideal sheaf corresponding to a formal subscheme $\widehat{S} \subset \widehat{X}$.

Since being an invariant divisor can be checked locally, it suffices to check $\widehat{S}$ is a formal invariant divisor in the case where $X$ is affine.

If $X$ is affine, then let $\widetilde{X}=\operatorname{Spec} \mathcal{O}_{\widehat{X}}$ and let $\widetilde{W}=W \times_{X} \widetilde{X}$ and let $\tilde{\pi}: \widetilde{W} \rightarrow \widetilde{X}$ be induced map. By the Grothendieck existence theorem, $\hat{S}^{\prime}$ corresponds to a closed subscheme of $\widetilde{W}$ denoted $\widetilde{S}^{\prime}$ and $\hat{S}$ correspond to a closed subscheme of $\widetilde{X}$ denoted $\widetilde{\widetilde{S}}$. The above construction gives us $\tilde{\pi}_{*} \widetilde{S}^{\prime}=\widetilde{S}$. Observe that $\widetilde{S}$ is a divisor on $\widetilde{X}$. Let $\widetilde{U}=\widetilde{X} \backslash \tilde{\pi}(\operatorname{Exc} \tilde{\pi})$ and note that $\widetilde{U}$ is a Zariski open subset and $\widetilde{S} \cap \widetilde{U} \neq \emptyset$. It follows immediately that $\widetilde{S} \cap \widetilde{U}$ is $\left.\mathcal{F}\right|_{\widetilde{U}^{-}}$ invariant (since $\tilde{\pi}$ is an isomorphism above $\widetilde{U}$ ). Since $\widetilde{S}$ admits an invariant Zariski dense subset we see that in fact $\widetilde{S}$ is invariant. The theorem on formal functions tells us that the completion of $\widetilde{S}$ along $V$ is exactly $\widehat{S}$, and our result follows.

\subsection{F-dlt foliated pairs}

Definition 3.6 Let $X$ be a normal variety and let $\mathcal{F}$ be a co-rank one foliation on $X$. Suppose that $K_{\mathcal{F}}+\Delta$ is $\mathbb{Q}$-Cartier.

We say $(\mathcal{F}, \Delta)$ is foliated divisorial $\log$ terminal (F-dlt) if

1. each irreducible component of $\Delta$ is generically transverse to $\mathcal{F}$ and has coefficient at most one, and

2. there exists a foliated $\log$ resolution $\pi: Y \rightarrow X$ of $(\mathcal{F}, \Delta)$ which only extracts divisors $E$ of discrepancy $>-\epsilon(E)$.

Remark 3.7 As we show in Remark 3.15, canonical singularities are not in general F-dlt. On the other hand, if $(\mathcal{F}, \Delta)$ is a F-dlt pair, then it is $\log$ canonical. Indeed, by assumption, there exists a foliated $\log$ resolution $\pi: Y \rightarrow X$ which only extract divisors $E$ of discrepancy $>-\epsilon(E)$. Thus, if $\mathcal{G}$ is the transformed foliation on $Y$ and $\Gamma$ is the strict transform of $\Delta$ in $Y$, then we may write

$$
K_{\mathcal{G}}+\Gamma+F=\pi^{*}\left(K_{\mathcal{F}}+\Delta\right)+G
$$


where $F, G \geq 0$ are $\pi$-exceptional $\mathbb{Q}$-divisors without any common component. Note, in particular, that no component of $\Gamma+F$ is $\mathcal{G}$-invariant and, therefore, Lemma 3.3 implies that $(\mathcal{G}, \Gamma+F)$ is $\log$ canonical. Thus, for any valuation $S$, we have

$$
a(S, \mathcal{F}, \Delta) \geq a(S, \mathcal{G}, \Gamma+F) \geq-\epsilon(S)
$$

and our claim follows.

Lemma 3.8 Let $X$ be a normal variety and let $\mathcal{F}$ be a co-rank one foliation on $X$. Suppose that $(\mathcal{F}, \Delta)$ is a $F$-dlt pair on $X$ and let $\pi: Y \rightarrow X$ be a foliated $\log$ resolution such that $a(E, \mathcal{F}, \Delta)>-\epsilon(E)$ for any $\pi$-exceptional divisor E.

Then $\pi$ is an isomorphism at the general point of $\pi^{-1}(W)$ for any lc centre $W \subset X$. In particular, $(\mathcal{F}, \Delta)$ is foliated log smooth at the generic point of $W$.

Proof Suppose by contradiction that $\pi$ is not an isomorphism at the general point of $\pi^{-1}(W)$. We may write

$$
K_{\mathcal{G}}+\Gamma=\pi^{*}\left(K_{\mathcal{F}}+\Delta\right)+F,
$$

where $\Gamma, F \geq 0$ are $\mathbb{Q}$-divisors without any common component and $\mathcal{G}$ is the pulled back foliation on $Y$. Note that $(\mathcal{G}, \Gamma)$ is $\log$ smooth.

By assumption, there exists a valuation $T$, whose centre in $X$ is $W$ and such that $a(T, \mathcal{F}, \Delta)=-\epsilon(T)$. Since $\pi$ is not an isomorphism at the general point of $\pi^{-1}(W)$ and since Exc $\pi$ is a divisor, there exists a $\pi$-exceptional prime divisor $E$ which contains the centre of $T$ in $Y$.

If $E$ is $\mathcal{G}$-invariant, then $E$ is contained in the support of $F$ and we have

$$
a(T, \mathcal{G}, \Gamma)<a(T, \mathcal{F}, \Delta)=-\epsilon(T),
$$

which contradicts Lemma 3.3.

Similarly, if $E$ is not $\mathcal{G}$-invariant, then $E$ is not contained in the support of $\lfloor\Gamma\rfloor$ and there exists $\delta>0$ such that if $\Gamma^{\prime}:=\Gamma+\delta E$ then, $\left(\mathcal{G}, \Gamma^{\prime}\right)$ is $\log$ smooth, the coefficients of $\Gamma^{\prime}$ are not greater than one and

$$
a\left(T, \mathcal{G}, \Gamma^{\prime}\right)<a(T, \mathcal{G}, \Gamma) \leq a(T, \mathcal{F}, \Delta)=-\epsilon(T),
$$

which contradicts again Lemma 3.3.

Proposition 3.9 Let $X$ be a normal variety and let $\mathcal{F}$ be a co-rank one foliation on $X$. Let $(\mathcal{F}, \Delta)$ be a F-dlt pair on $X$.

Then $(\mathcal{F}, \Delta)$ has only finitely many lc centres of codimension at least two, which are not contained in the support of $\lfloor\Delta\rfloor$. 
Proof By Lemma 3.8, we have that $(\mathcal{F}, \Delta)$ is foliated $\log$ smooth at the generic point of every lc centre. Let $Z \subset X$ be a subvariety of codimension at least two which is not contained in the support of $\lfloor\Delta\rfloor$ and such that $(\mathcal{F}, \Delta)$ is foliated $\log$ smooth at the generic point of $Z$. Let $\pi: Y \rightarrow X$ be the blow up at $Z$ with exceptional divisor $E$ and suppose that $Z \subset$ Supp $\Delta$. Computing as in Lemma 3.3, we see that the discrepancy of this blow up is $>-\epsilon(E)$. Computing inductively we see that every divisor dominating $Z$ has discrepancy $>-\epsilon(E)$.

Thus, every lc centre of $(\mathcal{F}, \Delta)$ not contained in Supp $\lfloor\Delta\rfloor$ must also be an lc centre of $(\mathcal{F}, 0)$. Keeping in mind that $\mathcal{F}$ has simple singularities at the general point of $Z$, a straightforward computation shows that the lc centres of $(\mathcal{F}, 0)$ are strata of Sing $\mathcal{F}$ and therefore there are only finitely many such centres.

Remark 3.10 As in the proof of Proposition 3.9, it follows that if $\mathcal{F}$ is a co-rank one foliation on a normal variety $X$ and $(\mathcal{F}, \Delta)$ is a $F$-dlt pair, then there are only finitely many lc centres which are transverse to $\mathcal{F}$. Indeed, as in the proof of Lemma 3.3, these centres are strata of $\lfloor\Delta\rfloor$.

Lemma 3.11 Let $X$ be a normal threefold and let $\mathcal{F}$ be a co-rank one foliation on $X$. Suppose that $(\mathcal{F}, \Delta)$ is a $F$-dlt pair on $X$ and that $\phi: X \rightarrow X^{\prime}$ is a sequence of steps of a $\left(K_{\mathcal{F}}+\Delta\right)$-MMP. Let $\left(\mathcal{F}^{\prime}, \Delta^{\prime}\right)$ be the transformed foliated pair on $X^{\prime}$.

Then $\left(\mathcal{F}^{\prime}, \Delta^{\prime}\right)$ is also F-dlt.

Proof We may assume that $\phi: X \rightarrow X^{\prime}$ is either a $\left(K_{\mathcal{F}}+\Delta\right)$-flip or a divisorial contraction. We denote by $\Sigma$ the flipped locus if $\phi$ is a flip and $\Sigma:=\phi(\operatorname{Exc} \phi)$ if $\phi$ is a divisorial contraction.

By assumption, there exists a foliated $\log$ resolution $\pi: Y \rightarrow X$ of $(\mathcal{F}, \Delta)$ which only extracts divisors $E$ of discrepancy $>-\epsilon(E)$. It is enough to show that $\left(\mathcal{F}^{\prime}, \Delta^{\prime}\right)$ also admits such a foliated $\log$ resolution. Let $\bar{Y} \subset Y \times X^{\prime}$ be the closure of the graph of $\phi \circ \pi$ and let $p: \bar{Y} \rightarrow Y$ be the induced proper birational morphism. Let $\mathcal{G}$ be the pulled back foliation on $\bar{Y}$ and let $\bar{\Delta}$ be the strict transform of $\Delta$ in $\bar{Y}$. Let $f=\pi \circ p: \bar{Y} \rightarrow X$ be the induced morphism and let $F:=\sum F_{i}$ where the sum runs over all the $f$-exceptional divisors. Let $g: Y^{\prime} \rightarrow \bar{Y}$ be a foliated $\log$ resolution of $(\mathcal{G}, \bar{\Delta}+F)$. We may assume that $g$ is an isomorphism in the locus where $(\mathcal{G}, \bar{\Delta}+F)$ is log smooth.

Let $\pi^{\prime}: Y^{\prime} \rightarrow X^{\prime}$ be the induced morphism, let $E^{\prime}$ be a prime $\pi^{\prime}$-exceptional divisor and let $W$ be the centre of $E^{\prime}$ in $X^{\prime}$. We claim that $a\left(E^{\prime}, \mathcal{F}^{\prime}, \Delta^{\prime}\right)>$ $-\epsilon\left(E^{\prime}\right)$. If $W$ is contained in $\Sigma$, then Lemma 2.7 and Remark 3.7 imply that

$$
-\epsilon\left(E^{\prime}\right) \leq a\left(E^{\prime}, \mathcal{F}, \Delta\right)<a\left(E^{\prime}, \mathcal{F}^{\prime}, \Delta^{\prime}\right)
$$


as claimed. If $W$ is not contained in $\Sigma$ then, by construction, the morphism $Y^{\prime} \rightarrow Y$ is an isomorphism at the general point of $E^{\prime}$ and the strict transform of $E^{\prime}$ in $Y$ is $\pi$-exceptional. Thus,

$$
-\epsilon\left(E^{\prime}\right)<a\left(E^{\prime}, \mathcal{F}, \Delta\right)=a\left(E^{\prime}, \mathcal{F}^{\prime}, \Delta^{\prime}\right),
$$

and, again, our claim follows.

Lemma 3.12 Let $X$ be a normal threefold and let $\mathcal{F}$ be a co-rank one foliation on $X$ with non-dicritical singularities. Let $C \subset X$ be a curve tangent to $\mathcal{F}$ and suppose that $(\mathcal{F}, \Delta)$ is a $F$-dlt pair on $X$.

Then

1. $(\mathcal{F}, \Delta)$ is canonical at the generic point of $C$.

2. If in addition $C \subset \operatorname{Sing} X$ then $(\mathcal{F}, \Delta)$ is terminal at the generic point of C.

Proof Item (1) follows from the observation that every divisor $E$ dominating $C$ on some $\log$ resolution must be foliation invariant.

If $(\mathcal{F}, \Delta)$ is not terminal along $C$ then $C$ is an lc centre. Thus, Lemma 3.8 implies that $X$ is smooth at the generic point of $C$, i.e. $C$ is not contained in Sing $X$, and (2) follows.

Remark 3.13 In fact, in Case (1) above there is an open set $U \subset X$ intersecting $C$ on which $(\mathcal{F}, \Delta)$ has canonical singularities. Indeed, let $\pi: X^{\prime} \rightarrow X$ be a foliated $\log$ resolution and let $E_{1}, \ldots, E_{k}$ be the $\pi$-exceptional divisors of discrepancy $<0$ with respect to $(\mathcal{F}, \Delta)$. Let $W=\pi\left(\sum E_{i}\right)$. By assumption $C$ is not contained in $W$ and observe that $(\mathcal{F}, \Delta)$ has canonical singularities on $U:=X \backslash W$.

Lemma 3.14 Let $X$ be a normal quasi-projective threefold and let $\mathcal{F}$ be a corank one foliation with non-dicritical singularities on $X$. Suppose that $(\mathcal{F}, \Delta)$ is $F$-dlt and let $C \subset X$ be a curve tangent to $\mathcal{F}$.

Let $\widehat{X}$ denote the formal completion of $X$ along $C$.

1. If $(\mathcal{F}, \Delta)$ is terminal at the generic point of $C$ there exists a single $\mathcal{F}$ invariant divisor $S \subset \widehat{X}$, and we may take $S$ to be convergent.

2. If $(\mathcal{F}, \Delta)$ is not terminal at the generic point of $C$ then $X$ is smooth at the generic point of $C$ and at a general point $P \in C$ there are 2 (formal) separatrices at $P$ containing $C$, each of which may be extended to a divisor $S \subset \widehat{X}$. Moreover, at least one of these separatrices may be extended to a convergent one.

Proof Note that Lemma 3.12 implies that $(\mathcal{F}, \Delta)$ is canonical at the generic point of $C$. Suppose that $(\mathcal{F}, \Delta)$ is terminal at the generic point of $C$. We 
first show the existence of a unique separatrix containing $C$ at a general point $P \in C$, and so we are free to shrink to an analytic neighborhood of $P$. Let $H$ be a germ of a general hypersurface at $P$ and let $\mathcal{F}_{H}$ be the restricted foliation. We claim that $K_{\mathcal{F}_{H}}$ is $\mathbb{Q}$-Cartier and $\mathcal{F}_{H}$ has terminal singularities (cf. [41, Lemma 8.7]). Indeed, let $\pi: X^{\prime} \rightarrow X$ be a $\log$ resolution of $\mathcal{F}$ and set $\mathcal{F}^{\prime}:=\pi^{-1} \mathcal{F}$ and $\Delta^{\prime}=\pi_{*}^{-1} \Delta$. Perhaps shrinking about $P$ we may assume that Exc $\pi$ is $\mathcal{F}^{\prime}$-invariant and the following hold:

(i) For a general choice of $H$ set $H^{\prime}:=\pi^{*} H$. Then the log pair $\left(X^{\prime}, \Delta^{\prime}+\right.$ Exc $\left.\pi+H^{\prime}\right)$ is simple normal crossing and so $\pi$ is in fact a log resolution of $(\mathcal{F}, \Delta+H)$.

(ii) We may write $K_{\mathcal{F}^{\prime}}+\Delta^{\prime}+H^{\prime}=\pi^{*}\left(K_{\mathcal{F}}+\Delta+H\right)+\sum_{i=1}^{k} a_{i} E_{i}$ where $E_{1}, \ldots, E_{k}$ are the $\pi$-exceptional divisors and $a_{i}>0$ for each $i$.

(iii) $\left.\left(K_{\mathcal{F}^{\prime}}+H^{\prime}\right)\right|_{H^{\prime}}=K_{\mathcal{F}_{H^{\prime}}}$ where $\mathcal{F}_{H^{\prime}}$ is the restricted foliation, see [41, Lemma 3.1 and Corollary 3.3] (note that we will prove a more general adjunction statement in Lemma 3.18). Moreover, since $\left(\mathcal{F}^{\prime}, \Delta^{\prime}+H^{\prime}\right)$ is foliated $\log$ smooth it follows that $\left(\mathcal{F}_{H^{\prime}},\left.\Delta^{\prime}\right|_{H}\right)$ is $\log$ smooth (c.f. the proof of Lemma 2.19).

Set $D_{i}=E_{i} \cap H$. By items (ii) and (iii) we see that $K_{\mathcal{F}_{H^{\prime}}}+\left.\Delta^{\prime}\right|_{H^{\prime}} \sim_{\mathbb{Q}}$ $\sum_{i=1}^{k} a_{i} D_{i}$. Let $r: H^{\prime} \rightarrow H^{\prime \prime} / H$ be a run of the $K_{\mathcal{F}_{H^{\prime}}}+\left.\Delta^{\prime}\right|_{H^{\prime}}$-MMP over $H$, see [41, Corollary 2.26]. We know that $H^{\prime \prime}$ has at worst quotient singularities and $\left(\mathcal{F}_{H^{\prime \prime}},\left.r_{*} \Delta^{\prime}\right|_{H^{\prime}}\right)$, and hence $\mathcal{F}_{H^{\prime \prime}}$, has terminal singularities where $\mathcal{F}_{H^{\prime \prime}}$ is the transform of $\mathcal{F}_{H}$. By the negativity lemma we see that $r_{*}\left(\sum_{i=1}^{k} b_{i} D_{i}\right)=0$, i.e., $H^{\prime \prime} \cong H$. Thus $\mathcal{F}_{H}$ has terminal singularities.

By [31, Corollary I.2.2] there is a unique germ of a convergent curve $P \in \gamma$ which is $\mathcal{F}_{H}$-invariant. In turn, $\gamma$ is contained in a unique convergent separatrix of $\mathcal{F}$ at $P$. Indeed, the unicity follows from the fact that $\gamma$ is the unique $\mathcal{F}_{H^{-}}$ invariant curve through $P$. The existence of this separatrix is guaranteed by [11, Main Theorem] when $\mathcal{F}$ has non-dicritical singularities and $X$ is smooth, the case where $\mathcal{F}$ has non-dicritical singularities and $X$ is singular follows by essentially the same arguments, see [41, Corollary 5.4] (note that the hypothesis on the compactness of the curve $C$ is not needed here). By Lemma 3.5 we may extend this separatrix to a convergent invariant divisor on $\widehat{X}$.

If $(\mathcal{F}, \Delta)$ is not terminal at the generic point of $C$, then $C$ is an lc centre and Lemma 3.8 implies that $(\mathcal{F}, \Delta)$ is $\log$ smooth at the general point of $C$. This implies then that $\mathcal{F}$ has simple singularities at a general point $P \in C$, and hence has two (possibly formal) separatrices at $P$. By Lemma 3.5 we may extend each of them to formal $\mathcal{F}$-invariant divisors on $\widehat{X}$. By Lemma 2.20 one of these separatrices is convergent and so may be extended to a convergent invariant divisor. 
Remark 3.15 1. Even if at a general point $P \in C$ there are two distinct separatrices, the invariant divisor we produce by extending them might be the same for each separatrix.

2. In general, a canonical non-terminal singularity may only admit a single separatrix formal or otherwise. Thus, canonical does not imply F-dlt.

3. However, log terminal does imply F-dlt (keeping in mind that in general canonical does not imply log terminal for foliations).

4. Recall that, by Remark 3.7, F-dlt implies log canonical.

\subsection{Adjunction}

Recall the following result:

Lemma 3.16 Let $X$ be a normal threefold, let $\mathcal{F}$ be a co-rank one foliation with non-dicritical singularities and let $(\mathcal{F}, \Delta)$ be a foliated pair on $X$. Let $D=\sum D_{i}$ be an $\mathcal{F}$-invariant divisor on $X$ and suppose that $D$ is $\mathbb{Q}$-Cartier.

Suppose that $(\mathcal{F}, \Delta)$ is log terminal (resp. log canonical). Then $(X, \Delta+D)$ is log terminal (resp. log canonical).

Suppose that $(\mathcal{F}, \Delta)$ is F-dlt and $\lfloor\Delta\rfloor=0$. Then $(X, \Delta+(1-\epsilon) D)$ is klt for all $\epsilon>0$.

Suppose that $(\mathcal{F}, \Delta)$ is $F$-dlt and each $D_{i}$ is smooth in codimension one (but perhaps $\lfloor\Delta\rfloor \neq 0)$. Then $(X, \Delta+D)$ is dlt.

Proof The first claim is [41, Lemma 8.14], see also [29, Proposition 3.11].

The second claim is a direct consequence of Lemma 3.8.

The final claim follows by recalling the third point in Remark 3.2 and thereby noting that if each $D_{i}$ is smooth in codimension one then a foliated $\log$ resolution of $(\mathcal{F}, \Delta)$ is also a $\log$ resolution of $(X, \Delta+D)$.

In fact, Lemma 3.16 remains true even in the following slightly more general set up. Let $X$ be a normal threefold with a foliated pair $(\mathcal{F}, \Delta)$ and let $V \subset X$ be a closed subvariety and let $\widehat{X}$ be the formal completion of $X$ along $V$ and let $\widehat{\mathcal{F}}$ be the restriction of $\mathcal{F}$ to $\widehat{X}$. Then under the hypotheses of Lemma 3.16 the conclusions of the Lemma still hold even if $D \subset \widehat{X}$ is a formal divisor which is $\widehat{\mathcal{F}}$-invariant. We remark that the third point of Remark 3.2 implies that a $\log$ resolution of $(\mathcal{F}, \Delta)$, perhaps followed by some further blow ups in Sing $\mathcal{F}$, is a $\log$ resolution of $(\widehat{X}, \Delta+D)$, and in particular $(\widehat{X}, \Delta+D)$ admits a resolution by blow ups in algebraic centres.

Remark 3.17 Let $X$ be a normal threefold, let $\mathcal{F}$ be a co-rank one foliation on $X$, and let $P \in X$ be a point. Let $U$ be an analytic neighbourhood of $P$ and let $\mathcal{F}_{U}$ be the foliation restricted to $U$. Then, thanks to the existence of a $\log$ resolution and since simple singularities are canonical by Lemma 2.9, we 
know that if $\mathcal{F}$ is $(\log )$ canonical then $\mathcal{F}_{U}$ is $(\log )$ canonical. Conversely, if $\mathcal{F}_{U}$ is $(\log )$ canonical at $P$ then the same is true for $\mathcal{F}$.

Lemma 3.18 (Adjunction) Let $X$ be a normal threefold, let $\mathcal{F}$ be a co-rank one foliation on $X$. Let $(\mathcal{F}, \epsilon(S) S+\Delta)$ be a foliated pair where $S$ is a prime divisor and $\Delta \geq 0$ is a $\mathbb{Q}$-divisor on $X$ which does not contain $S$ in its support. Let $v: S^{v} \rightarrow S$ be the normalisation and let $\mathcal{G}$ be the restricted foliation to $S^{v}$ if $S$ is not $\mathcal{F}$-invariant and let $\mathcal{G}=T_{S^{\nu}}$ if $S$ is $\mathcal{F}$-invariant.

Then, there exists $\Theta \geq 0$ such that

$$
\left.\left(K_{\mathcal{F}}+\epsilon(S) S+\Delta\right)\right|_{S^{\nu}}=K_{\mathcal{G}}+\Theta .
$$

Now suppose that $K_{X}, K_{X}+\Delta$ and $S$ are $\mathbb{Q}$-Cartier and that $\mathcal{F}$ admits non-dicritical singularities. Then the following hold:

1. Suppose $\epsilon(S)=1$. Suppose moreover that $(\mathcal{F}, S+\Delta)$ is lc (resp. lt, resp. $F$-dlt). Then $(\mathcal{G}, \Theta)$ is lc (resp. lt, resp. F-dlt).

2. Suppose $\epsilon(S)=0$. Suppose that $\mathcal{F}$ is $F$-dlt. Then $\left(S^{\nu}, \Theta^{\prime}:=\lfloor\Theta\rfloor\right.$ red $\left.+\{\Theta\}\right)$ is $\log$ canonical and if, in addition, $S$ and Sing $\mathcal{F} \cap S$ are normal then $\left(S, \Theta^{\prime}\right)$ is dlt. Moreover, if $(\mathcal{F}, \Delta)$ is log terminal, then $\left(S^{\nu}, \Theta^{\prime}\right)$ is $\log$ terminal.

Proof The first claim follows from [41, Proposition 3.4] if $S$ is not $\mathcal{F}$-invariant and [2, Definition 3.11] if $S$ is $\mathcal{F}$-invariant. Note that [2, Definition 3.11] assumes that $\mathcal{F}$ has algebraic leaves, however the arguments work equally well in this situation.

We now prove the second claim. Let $\pi: Y \rightarrow X$ be a foliated log resolution of $(\mathcal{F}, \epsilon(S) S+\Delta)$, let $\mathcal{F}_{Y}$ be the pulled back foliation on $Y$ and let $T$ be the strict transform of $S$ in $Y$. If $(\mathcal{F}, \epsilon(S) S+\Delta)$ is F-dlt, then we choose $\pi$ so that $a(E, \mathcal{F}+\epsilon(S) S, \Delta)>-\epsilon(E)$ for any $\pi$-exceptional divisor $E$.

We may write

$$
K_{\mathcal{F}_{Y}}+\epsilon(T) T+\Delta^{\prime}+\sum a_{i} E_{i}=\pi^{*}\left(K_{\mathcal{F}}+\epsilon(S) S+\Delta\right),
$$

where $\Delta^{\prime}$ is the strict transform of $\Delta$ in $Y, a_{i} \in \mathbb{Q}$ and the sum is taken over all the $\pi$-exceptional divisors. Suppose first that $\epsilon(S)=1$ and note that, by the second point of Remark 3.2 we have that $T$ is generically transverse to $\mathcal{F}_{Y}$ and is not tangent to $\mathcal{F}_{Y}$ along any curves contained in $T$ and contains no onedimensional components of Sing $\mathcal{F}_{Y}$. Let $v^{\prime}: T^{v} \rightarrow T$ be the normalisation. By [41, Corollary 3.3] we get $\left.\left(K_{\mathcal{F}_{Y}}+T\right)\right|_{T^{v}}=K_{\mathcal{G}_{T}}+\Theta$ where $\mathcal{G}_{T}$ is the restricted foliation to $T^{v}$ and $\Theta \geq 0$ is a $\mathbb{Q}$-divisor. Note that the image of the support of $\Theta$ in $T$ is contained in curves where $T$ is tangent to $\mathcal{F}_{Y}$ and on one-dimensional components $\operatorname{Sing} \mathcal{F}_{Y} \cap T$. By our previous observation, we 
see that $\Theta=0$. Thus, we get

$$
K_{\mathcal{G}_{T}}+\left.\Delta^{\prime}\right|_{T^{v}}+\left.\sum a_{i} E_{i}\right|_{T^{v}}=\phi^{*}\left(K_{\mathcal{G}}+\Theta\right),
$$

where $\phi: T^{v} \rightarrow S^{v}$ is the induced morphism. By assumption $a_{i} \leq \epsilon\left(E_{i}\right)$ (resp. $\left.a_{i}<\epsilon\left(E_{i}\right)\right)$. To prove our result we need to show that $a_{i} \leq \epsilon\left(\left.E_{i}\right|_{T^{v}}\right)$ (resp. $a_{i}<\epsilon\left(\left.E_{i}\right|_{T^{v}}\right)$ ).

Suppose for sake of contradiction that for some $i$, we have $\epsilon\left(E_{i}\right)=1$, $\epsilon\left(\left.E_{i}\right|_{T^{v}}\right)=0$ and $a_{i}>0$ (resp. $a_{i} \geq 0$ ). In this case, consider the blow up of $Y$ at $E_{i} \cap T$ and let $F$ be the exceptional divisor. Notice that $\epsilon(F)=0$. However, this is a blow up of discrepancy $\leq-a_{i}<\epsilon(F)$ (resp. $\leq-a_{i} \leq \epsilon(F)$, resp. $\left.\leq-a_{i}<\epsilon(F)\right)$, and so we see that $\left(\mathcal{F}_{Y}, T+\Delta^{\prime}+\sum a_{i} E_{i}\right)$ is not lc (resp. 1t, resp. F-dlt), hence $(\mathcal{F}, S+\Delta)$ is not lc (resp. lt, resp. F-dlt). Thus, (1) follows.

Suppose now that $\epsilon(S)=0$ and let $\widehat{X}$ be the formal completion of $X$ along $S$. Let $T$ be the sum of all the (formal) invariant divisors meeting $S$, and which are not equal to $S$. Suppose for the moment that $T$ is $\mathbb{Q}$-Cartier. Observe that if Sing $\mathcal{F} \cap S$ is normal then each component of $T$ is smooth in codimension one. Then by Lemma 3.16 we know that $(\widehat{X}, S+T+\Delta)$ is dlt, furthermore, as in the proof of [41, Lemma 8.9], it follows that the different of $(\widehat{X}, S+T+\Delta)$ with respect to $S$ is exactly $\Theta^{\prime}$. We then apply adjunction for varieties to conclude.

We now handle the case where $T$ is not necessarily $\mathbb{Q}$-Cartier. By Lemma 3.16 and since $(\mathcal{F}, \Delta)$ is F-dlt, it follows that $X$ is klt. Suppose for sake of contradiction that there exists a point $P \in \widehat{X}$ such that $T$ is not $\mathbb{Q}$-Cartier at $P$. Then, since $X$ is klt, if $U$ is a small analytic neighbourhood of $P \in X$ we may find a small $\mathbb{Q}$-factorialisation $f: Y \rightarrow U$ such that $Y$ is analytically $\mathbb{Q}$-factorial by Remark 2.24. Let $\Sigma=f^{-1}(P)$, let $\widehat{U}$ be the formal completion of $U$ at $P$ and let $\widehat{Y}$ be the formal completion along $\Sigma$ so that we have a morphism $\hat{f}: \widehat{Y} \rightarrow \widehat{U}$.

Observe that $K_{Y}=f^{*} K_{U}$ and if we let $S^{\prime}=f^{*} S$ then $\Sigma \subset S^{\prime}$. Let $\widehat{S}^{\prime}$ denote the restriction of $S^{\prime}$ to $\widehat{Y}$. Let $\Sigma_{0}$ be a component of $\Sigma$ which meets $\hat{f}_{*}^{-1} T$. Note that $K_{Y} \cdot \Sigma_{0}=0$ and that, after possibly shrinking $U$, we may assume that $\Sigma_{0}$ spans an extremal ray in $\overline{N E}(Y / U)$. Thus, there exists a flop of $\Sigma_{0}$ over $U$, call it $\psi: Y \rightarrow Y^{\prime} / U$ and let $\Sigma_{0}^{\prime}$ be the flopped curve. Observe that $\Sigma_{0} \subset \hat{f}_{*}^{-1} T$ or $\Sigma_{0}^{\prime} \subset \psi_{*} \hat{f}_{*}^{-1} T$. So up to replacing $Y$ by $Y^{\prime}$, we may assume that $\Sigma_{0} \subset \hat{f}_{*}^{-1} T$. Next, note that $\Sigma_{0} \subset \widehat{S}^{\prime} \cap \hat{f}_{*}^{-1}(T) \subset$ Sing $\mathcal{H}$ where $\mathcal{H}:=f^{-1} \mathcal{F}$ and the latter inclusion holds because $S^{\prime}$ and $\hat{f}_{*}^{-1}(T)$ are both $\mathcal{H}$-invariant.

By Remark 3.7, the foliation $\mathcal{F}$ has $\log$ canonical singularities. Thus, since $f$ is small, $\mathcal{H}$ has also log canonical singularities. Note also by Lemma 3.14 that $\mathcal{H}$ has canonical, and not terminal, singularities at the generic point of $\Sigma_{0}$, since $\Sigma_{0}$ is contained in the intersection of two (formal) invariant divisors. Thus we may find a birational morphism $b: Y^{\prime} \rightarrow Y$ which extracts a divisor 
$E$ dominating $\Sigma_{0}$ such that $0=a(E, \mathcal{H})=a(E, \mathcal{F})$. Next, observe that $a(E, \mathcal{F}) \geq a(E, \mathcal{F}, \Delta) \geq-\epsilon(E)=0$ and so all these inequalities must be equalities and it follows that $P$ is a lc centre of $(\mathcal{F}, \Delta)$. By Lemma 3.8, $X$ is smooth at $P$, and so $T$ is necessarily Cartier at $P$, a contradiction. Thus, (2) follows.

Remark 3.19 Note that, using the same proof, the first part of Lemma 3.18 can be generalised to the formal setting. More specifically, let $X$ be a normal threefold and let $\mathcal{F}$ be a co-rank one foliation with non-dicritical singularities on $X$. Suppose that $(\mathcal{F}, \Delta)$ is a F-dlt pair on $X$, let $S$ be an $\mathcal{F}$-invariant divisor, let $\widehat{X}$ be the formal completion of $X$ along $S$ and let $v: S^{\nu} \rightarrow S$ be the normalisation. Then, we may write

$$
\left.\left(K_{\mathcal{F}}+\Delta\right)\right|_{S^{\nu}}=K_{S^{\nu}}+\Theta,
$$

where $\Theta \geq 0$ is a $\mathbb{Q}$-divisor on $S^{\nu}$.

Corollary 3.20 Let $X$ be a normal threefold and let $\mathcal{F}$ be a co-rank one foliation with non-dicritical singularities on $X$. Suppose that $(\mathcal{F}, \Delta)$ is a $F$ dlt pair on $X$, let $S$ be an $\mathcal{F}$-invariant divisor such that $(X, \Delta+S)$ is a log pair, let $\widehat{X}$ be the formal completion of $X$ along $S$ and let $v: S^{v} \rightarrow S$ be the normalisation. Let $T_{1}, \ldots, T_{k}$ be the collection of $\mathcal{F}$-invariant divisors on $\widehat{X}$ not equal to $S$ and suppose that $T=\sum T_{i}$ is $\mathbb{Q}$-Cartier. Write

$$
\left.\left(K_{\mathcal{F}}+\Delta\right)\right|_{S^{\nu}}=K_{S^{\nu}}+\Theta
$$

and let $\Theta^{\prime}$ be the different of $(\widehat{X}, \Delta+S+T)$ with respect to $S$.

Then $\Theta^{\prime} \leq \Theta$ with equality along the curves contained in Sing X. Moreover, for any irreducible curve $C$ in $S$, at the general point of which $\mathcal{F}$ has simple singularities and along which $S$ is a strong separatrix, the coefficients of $\Theta$ and $\Theta^{\prime}$ coincide.

Proof If $C$ is a curve contained in the singular locus of $S$, then $C \subset$ Sing $\mathcal{F} \cup$ Sing $X$. Since $T_{1}, \ldots, T_{k}$ are $\mathcal{F}$-invariant, it follows that $\Theta$ and $\Theta^{\prime}$ are supported on $v^{-1}(\operatorname{Sing} \mathcal{F} \cup \operatorname{Sing} X)$.

Let $C$ be an irreducible curve in $S^{\nu}$. By Lemma 3.12, we see that if $v(C) \subset$ Sing $X \cap S$ then $\mathcal{F}$ is terminal at the generic point of $v(C)$. In this case, by [41, Lemma 8.9], it follows that the coefficient of $C$ in $\Theta$ and $\Theta^{\prime}$ is the same.

So we may assume that $X$ is smooth at the generic point of $C$. If $v(C) \subset$ Sing $\mathcal{F} \cap S$ then $C$ is an lc center of $\mathcal{F}$ and so by Lemma 3.8, $\mathcal{F}$ has simple singularities at a general point of $C$. It follows that $S+T$ is a normal crossings divisor at the generic point of $C$ and so the coefficient of $C$ in $\Theta^{\prime}$ is equal to 1 .

Let $\omega$ be a 1-form defining $\mathcal{F}$ at a general point of $C$ and let $\omega_{S}$ be the restriction of $\omega$ to $S$. The coefficient of $C$ in $\Theta$ is the order of the zero of $\omega_{S}$ 
along $C$, in particular, it is an integer $\geq 1$. When $S$ is the strong separatrix along $C$ then the order of the zero of $\omega_{S}$ along $C$ is equal to 1 .

Remark 3.21 We emphasise that in Corollary 3.20 we are not assuming that $X$ is quasi-projective or that $S$ is an algebraic variety. Later on we will apply this Lemma in the case where $S$ is an analytic divisor on an analytic neighborhood of a proper curve $C$ contained in a quasi-projective threefold.

Lemma 3.22 Let $X$ be a $\mathbb{Q}$-factorial quasi-projective threefold and let $\mathcal{F}$ be a co-rank 1 foliation with non-dicritical singularities on $X$. Suppose that $(\mathcal{F}, \Delta)$ is $F$-dlt. Let $C$ be a one-dimensional lc centre of $(\mathcal{F}, \Delta)$ tangent to $\mathcal{F}$.

Then there exists $\Theta \geq 0$ such that

$$
\left.\left(K_{\mathcal{F}}+\Delta\right)\right|_{C^{v}}=K_{C^{\nu}}+\Theta,
$$

where $v: C^{v} \rightarrow C$ is the normalisation. Moreover, if $P$ is contained in the support of $\lfloor\Theta\rfloor$ then $v(P)$ is an lc centre of $(\mathcal{F}, \Delta)$.

Proof By Lemma 3.8, it follows that $(\mathcal{F}, \Delta)$ is log smooth at the general point of $C$. By Lemma 3.14, there exists a germ of an invariant divisor $S$ containing $C$ and if $C \subset \operatorname{Sing} \mathcal{F}$, then we may choose $S$ so that it is the strong separatrix at a general point of $C$. Let $v^{\prime}: S^{\nu} \rightarrow S$ be the normalisation of $S$ and set $C^{\prime}=v^{\prime-1}(C)$. Then it is easy to check that

$$
\left.\left(K_{\mathcal{F}}+\Delta\right)\right|_{S^{v}}=K_{S^{v}}+C^{\prime}+\Delta_{S^{v}}
$$

for some $\mathbb{Q}$-divisor $\Delta_{S^{v}} \geq 0$, whose support does not contain $C$.

Note that we have a morphism $C^{v} \rightarrow C^{\prime}$ and so we may write $\left(K_{S^{v}}+\right.$ $\left.C^{\prime}+\Delta_{S^{v}}\right)\left.\right|_{C^{v}}=K_{C^{v}}+\Theta$. Thus, by adjunction for varieties, the image of the non-klt locus of $\left(C^{\nu}, \Theta\right)$ is supported on the non-klt locus of $\left(S^{\nu}, C^{\prime}+\Delta_{S^{\nu}}\right)$. We claim these centres are contained in lc centres of $(\mathcal{F}, \Delta)$. Let $P \in S$, by Lemma 3.18 we see that if $(\mathcal{F}, \Delta)$ is $\log$ terminal at $P$ then $\left(S^{v}, C^{\prime}+\Delta_{S^{v}}\right)$ must also be $\log$ terminal at $P$, and so every point in the non-klt locus of $\left(S^{\nu}, C^{\prime}+\Delta_{S^{\nu}}\right)$ is an lc centre of $(\mathcal{F}, \Delta)$.

Lemma 3.23 Let $X$ be a normal threefold and let $\mathcal{F}$ be a corank one foliation with non-dicritical singularities. Let $S$ be an irreducible divisor transverse to $\mathcal{F}$ and suppose that $K_{X}+\Delta+S$ and $K_{\mathcal{F}}+\Delta+S$ are $\mathbb{Q}$-Cartier where $\Delta \geq 0$ and $S$ is not contained in the support of $\Delta$. Write $n^{*}\left(K_{X}+\Delta+S\right)=K_{S^{n}}+\Theta$ and $n^{*}\left(K_{\mathcal{F}}+\Delta+S\right)=K_{\mathcal{F}_{S}}+\Theta^{\prime}$ where $n: S^{n} \rightarrow S$ is the normalisation. Let $C \subset S$ be a curve such that $n(C)$ is transverse to $\mathcal{F}$.

Then the coefficient of $C$ in $\Theta$ is equal to the coefficient of $C$ in $\Theta^{\prime}$.

Proof By [41, Lemma 3.11] we have that the usual discrepancy of any divisor dominating $n(C)$ is equal to the foliated discrepancy. By construction, the 
coefficient of the different along $C$ is only a function of the discrepancies of divisors dominating $C$ with respect to the pairs $(X, \Delta+S)$ and $(\mathcal{F}, \Delta+S)$ and our claimed equality follows.

\subsection{Bertini type results}

Lemma 3.24 Let $X$ be a normal projective threefold and let $\mathcal{F}$ be a co-rank one foliation on $X$ with non-dicritical singularities. Let $(\mathcal{F}, \Delta)$ be a $F$-dlt pair such that $\lfloor\Delta\rfloor=0$ and let $A$ be an ample $\mathbb{Q}$-divisor on $X$.

Then there exists an effective $\mathbb{Q}$-divisor $A^{\prime} \sim_{\mathbb{Q}} A$ such that

1. $\left(\mathcal{F}, \Delta+A^{\prime}\right)$ is also F-dlt;

2. $\left\lfloor\Delta+A^{\prime}\right\rfloor=0$; and

3. the support of $A^{\prime}$ does not contain any lc centre of $(\mathcal{F}, \Delta)$.

Proof Lemma 3.8 implies that $(\mathcal{F}, \Delta)$ admits a foliated $\log$ resolution $\pi: Y \rightarrow$ $X$ which only extracts divisors $E$ of discrepancy $>-\epsilon(E)$ and which is an isomorphism at the general point of $\pi^{-1}(W)$ for any $W \subset X$ which is a lc centre of $(\mathcal{F}, \Delta)$. We claim that there exists a sufficiently divisible positive integer $m$ such that if $H \in|m A|$ is a general element and $A^{\prime}:=\frac{1}{m} H$ then

(i) the support of $A^{\prime}$ does not contain any lc centre of $(\mathcal{F}, \Delta)$;

(ii) each irreducible component of $A^{\prime}$ is generically transverse to $\mathcal{F}$ and $\lfloor\Delta+$ $\left.A^{\prime}\right\rfloor=0$;

(iii) if $x \in X$ is the general point of a $\log$ canonical centre of $(\mathcal{F}, \Delta)$ then, $\left(\mathcal{F}, \Delta+A^{\prime}\right)$ is $\log$ smooth in a neighbourhood of $x$; and

(iv) if $(\mathcal{F}, \Delta)$ is klt in a neighbourhood of $x \in X$ then $\left(\mathcal{F}, \Delta+A^{\prime}\right)$ is also klt in a neighbourhood of $x$.

Assuming the claim, let $\mathcal{G}:=\pi^{-1} \mathcal{F}$. We may write

$$
K_{\mathcal{G}}+\Gamma=\pi^{*}\left(K_{\mathcal{F}}+\Delta\right)
$$

for some $\mathbb{Q}$-divisor $\Gamma$ on $Y$. Then there exists a foliated $\log$ resolution $\pi^{\prime}: Y^{\prime} \rightarrow$ $Y$ of $\left(\mathcal{G}, \Gamma+\pi^{*} A^{\prime}\right)$ such that if $p: Y^{\prime} \rightarrow X$ is the induced morphism, then $\pi^{\prime}$ is an isomorphism at the general point of $p^{-1}(W)$ for any lc centre $W$ of $(\mathcal{F}, \Delta)$. Thus, $a\left(F, \mathcal{F}, \Delta+A^{\prime}\right)>-\epsilon(F)$ for any prime divisor $F$ on $Y^{\prime}$ which is $p$-exceptional and the Lemma follows.

We now prove the claim. Since $\lfloor\Delta\rfloor=0$, Proposition 3.9 implies that if $m$ is sufficiently divisible then $H$ does not contain any lc centre of $(\mathcal{F}, \Delta)$. Thus, (i) holds. It is easy to check that (ii) holds.

Now we check (iii). Let $W$ be a $\log$ canonical centre of $(\mathcal{F}, \Delta)$. By Lemma 3.8, $(\mathcal{F}, \Delta)$ is foliated log smooth at the generic point of $W$. In particular, $\mathcal{F}$ admits simple singularities at the general point of $W$. If $x \in \operatorname{Sing} \mathcal{F}$ is 
an isolated point, then we may choose $m$ sufficiently divisible so that $x$ is not contained in the support of $H$. Similarly, we may assume that $x$ is the general point of a one-dimensional component $C$ of Sing $\mathcal{F}$. Thus, we may choose $m$ so that $H$ is transverse to $C$ and, in particular, if $\Sigma_{1}, \Sigma_{2}$ are, possibly formal, $\mathcal{F}$-invariant divisors passing through $x$, then $C$ is contained in $\Sigma_{1} \cap \Sigma_{2}$ and $\left(X, \Sigma_{1}+\Sigma_{2}+H\right)$ is a normal crossing pair at $x$. It follows that $\left(\mathcal{F}, \Delta+A^{\prime}\right)$ is $\log$ smooth at $x$. Therefore (iii) holds.

Finally, we check (iv). First, let $U:=X \backslash \operatorname{Sing} X$ and let $x \in U$ be a point such that $(\mathcal{F}, \Delta)$ is klt in a neighborhood of $x$. If $x \notin H$ then there is nothing to prove, and if $x \in H$ and $H$ is general we may also assume that $x \in U \backslash \operatorname{Sing} \mathcal{F}$. Moreover, if $H$ is sufficiently general then by [3, Lemma 2.9] it follows that $(\mathcal{F}, H)$ is $\log$ smooth on $U \backslash \operatorname{Sing} \mathcal{F}$, except perhaps at finitely many points, $P_{1}, \ldots, P_{m}$. In fact a dimension count as in [3, Lemma 2.9] shows that a general choice of $H$ will have at worst an order 2 tangency with $\mathcal{F}$ at $P_{i}$, i.e., in some local analytic coordinates $(x, y, z)$ we have $\mathcal{F}$ is defined by $d z$ and $H=\left\{z+x^{2}+y^{2}=0\right\}$. In particular, at the $P_{i}$ we know that $\left(\mathcal{F}, \frac{1}{4} H\right)$ is klt. Note that $\pi$ is a foliated $\log$ resolution of $(\mathcal{F}, \Delta+H)$ along $U$ except at $P_{1}, \ldots, P_{m}$. It then follows that for $m$ sufficiently divisible and $H$ sufficiently general, we have that $\left(\mathcal{F}, \Delta+A^{\prime}\right)$ is klt on $U \backslash \operatorname{Sing} \mathcal{F}$.

Next, write Sing $X=Z_{0} \cup Z_{1}$ where $Z_{0}$ are the components of Sing $X$ which are tangent to $\mathcal{F}$ and $Z_{1}$ are the components which are transverse. Suppose that $x \in Z_{0}$. Observe that $\pi^{-1}\left(Z_{0}\right)$ is by definition $\mathcal{G}$-invariant and so for a general choice of $H$, we know that $\left(\mathcal{G}, \Gamma+\pi^{*} H\right)$ is foliated log smooth in a neighbourhood of $\pi^{-1}(x)$. It therefore follows immediately that, for $m$ sufficiently divisible, $\left(\mathcal{F}, \Delta+A^{\prime}\right)$ is klt in a neighbourhood of $x$.

Finally suppose that $x \in Z_{1}$. Without loss of generality we may assume that $x$ is a general point of $Z_{1}$. By non-dicriticality we know that $\pi^{-1}(x)$ is tangent to $\mathcal{G}$. In fact, arguing as in the proof of Lemma 2.14 we see that $\mathcal{G}$ is smooth on a neighbourhood of $\pi^{-1}(x)$ and so by Lemma 3.5 it follows that there exists a $\mathcal{G}$-invariant divisor $S$ defined on an analytic neighbourhood of $\pi^{-1}(x)$ such that $\pi^{-1}(x) \subset S$.

We claim that $\left(\mathcal{G}, \Gamma+\pi^{*} A^{\prime}\right)$ is klt in a neighbourhood of $\pi^{-1}(x)$ if and only if $\left(Y, \Gamma+S+\pi^{*} A^{\prime}\right)$ is log terminal in a neighbourhood of $\pi^{-1}(x)$. Supposing the claim, then we are done by observing that if $\left(X, \Delta+\pi_{*} S\right)$ is log terminal at $x$ then $\left(X, \Delta+\pi_{*} S+A^{\prime}\right)$ is $\log$ terminal at $x$ for sufficiently divisible $m$ and sufficiently general $H$.

We now prove the above claim. After possibly shrinking $x$, we may consider a $\log$ resolution $\mu: \widetilde{Y} \rightarrow Y$ of $\left(Y, \Gamma+S+\pi^{*} A^{\prime}\right)$ which is an isomorphism away from $(\pi \circ \mu)^{-1}(x)$. Let $E$ be the support of Exc $\mu$. Then $K_{\mu^{-1} \mathcal{G}}$ and $K_{\widetilde{Y}}+\mu_{*}^{-1} S+E$ are numerically equivalent over $Y$. Indeed, if $E_{0}$ is any component of $E$ then, since $\mu_{*}^{-1} S \cap E_{0}, E^{\prime} \cap E_{0} \subset \operatorname{Sing} \mu^{-1} \mathcal{G}$ where $E^{\prime}$ is 
any other component of $E$, Corollary 3.20 implies that

$$
\left.K_{\mu^{-1} \mathcal{G}}\right|_{E_{0}}=K_{E_{0}}+\left.\left(E-E_{0}+\mu_{*}^{-1} S\right)\right|_{E_{0}} .
$$

We also have

$$
\left.\left(K_{\widetilde{Y}}+E+\mu_{*}^{-1} S\right)\right|_{E_{0}}=K_{E_{0}}+\left.\left(E-E_{0}+\mu_{*}^{-1} S\right)\right|_{E_{0}} .
$$

It follows that the $\log$ discrepancies of $\left(Y, \Gamma+S+\pi^{*} A^{\prime}\right)$ are equal to the discrepancies of $\left(\mathcal{G}, \Gamma+\pi^{*} A^{\prime}\right)$ as required. Therefore (iv) holds.

Corollary 3.25 Set up as in Lemma 3.24. Suppose in addition that $(\mathcal{F}, \Delta)$ is log smooth and has canonical singularities.

Then there exists an effective $\mathbb{Q}$-divisor $A^{\prime} \sim_{\mathbb{Q}} A$ such that $\left(\mathcal{F}, \Delta+A^{\prime}\right)$ has canonical singularities.

Proof By Lemma 3.24 we may find an effective $\mathbb{Q}$-divisor $A^{\prime} \sim_{\mathbb{Q}} A$ so that $\left(\mathcal{F}, \Delta+A^{\prime}\right)$ is F-dlt, hence log canonical by Remark 3.7. It suffices to show that for any divisor $E$ on some model of $X$ that $a\left(E, \mathcal{F}, \Delta+A^{\prime}\right) \geq 0$. Clearly this holds for all foliation invariant divisors, so let $E$ be one such divisor which is not foliation invariant and let $W$ be the centre of $E$ on $X$.

Since $\mathcal{F}$ has non-dicritical singularities, by Remark 2.13 we know that $W$ is necessarily transverse to $\mathcal{F}$. In particular, by [41, Lemma 3.11], we see that the foliation discrepancies for divisors centred over $W$ are equal to the classical discrepancies.

If $A^{\prime}$ is sufficiently general with sufficiently small coefficients then we know that $\left(X, \Delta+A^{\prime}\right)$ has canonical singularities at the generic point of $W$, and so $\left(\mathcal{F}, \Delta+A^{\prime}\right)$ has canonical singularities at the generic point of $W$, which implies our claim.

Lemma 3.26 Let $X$ be a $\mathbb{Q}$-factorial projective threefold and let $\mathcal{F}$ be a corank one foliation on $X$ with non-dicritical singularities. Let $\Delta=A+B$ be $a \mathbb{Q}$-divisor such that $(\mathcal{F}, \Delta)$ is a $F$-dlt pair, $A \geq 0$ is an ample $\mathbb{Q}$-divisor and $B \geq 0$.

Then there exist $\mathbb{Q}$-divisors $A^{\prime}, B^{\prime} \geq 0$ such that $A^{\prime}$ is ample and if $\Delta^{\prime}=$ $A^{\prime}+B^{\prime}$ then

1. $\Delta^{\prime} \sim_{\mathbb{Q}} \Delta$,

2. $\left\lfloor\Delta^{\prime}\right\rfloor=0$,

3. $\left(\mathcal{F}, \Delta^{\prime}\right)$ is $F$-dlt and

4. if $E$ is a valuation such that $a\left(E, \mathcal{F}, \Delta^{\prime}\right)=-\epsilon(E)$ then $\epsilon(E)=0$ and $a(E, \mathcal{F})=0$. 
Proof Let $\delta>0$ be sufficiently small rational number, so that $A+\delta B$ is ample and let $B^{\prime}:=(1-\delta) B$. By Lemma 3.24, there exists a $\mathbb{Q}$-divisor

$$
0 \leq A^{\prime} \sim_{\mathbb{Q}} A+\delta B
$$

such that, if $\Delta^{\prime}:=(1-\delta) B+A^{\prime}$ then (1), (2) and (3) hold and the support of $A^{\prime}$ does not contain any lc centre of $(\mathcal{F},(1-\delta) B)$. Thus, if $E$ is a valuation such that $a\left(E, \mathcal{F}, \Delta^{\prime}\right)=-\epsilon(E)$ then $a(E, \mathcal{F},(1-\delta) B)=-\epsilon(E)$. Since

$$
a(E, \mathcal{F}, B) \geq a(E, \mathcal{F}, \Delta) \geq-\epsilon(E),
$$

it follows that the centre $W$ of $E$ in $X$ is not contained in the support of $B$ and, in particular, $a(E, \mathcal{F})=-\epsilon(E)$. By Lemma 3.8, we have that $\mathcal{F}$ has simple singularities along the generic point of $W$. As in the proof of Lemma 2.9, it follows that $\epsilon(E)=0$. Thus, (4) follows.

Lemma 3.27 Let $X$ be a $\mathbb{Q}$-factorial projective threefold and let $\mathcal{F}$ be a corank one foliation on $X$ with non-dicritical singularities. Let $\Delta=A+B$ be $a \mathbb{Q}$-divisor such that $(\mathcal{F}, \Delta)$ is a $F$-dlt pair, $A \geq 0$ is an ample $\mathbb{Q}$-divisor and $B \geq 0$. Let $\varphi: X \rightarrow X^{\prime}$ be a sequence of steps of the $\left(K_{\mathcal{F}}+\Delta\right)-M M P$ and let $\mathcal{F}^{\prime}$ be the transformed foliation on $X^{\prime}$.

Then, there exist $\mathbb{Q}$-divisors $A^{\prime} \geq 0$ and $C^{\prime} \geq 0$ on $X^{\prime}$ such that

1. $\varphi_{*} A \sim \mathbb{Q} A^{\prime}+C^{\prime}$,

2. $A^{\prime}$ is ample, and

3. if $\Delta^{\prime}:=A^{\prime}+C^{\prime}+\varphi_{*} B$ then $\Delta^{\prime} \sim_{\mathbb{Q}} \varphi_{*} \Delta$ and $\left(\mathcal{F}^{\prime}, \Delta^{\prime}\right)$ is $F$-dlt.

Proof By Lemma 3.26, we may assume that $\lfloor\Delta\rfloor=0$. We may also assume that $\varphi: X \rightarrow X^{\prime}$ is a $\left(K_{\mathcal{F}}+\Delta\right)$-flip (resp. $\left(K_{\mathcal{F}}+\Delta\right)$-divisorial contraction). Let $H \geq 0$ be a general ample $\mathbb{Q}$-divisor on $X^{\prime}$. After possibly replacing $H$ by a smaller multiple, we may assume that if $H_{X}$ is the strict transform of $H$ in $X$ then $A-H_{X}$ is ample. In particular, by Lemma 3.24, there exists an effective $\mathbb{Q}$-divisor $C \sim_{\mathbb{Q}} A-H_{X}$ and $\epsilon>0$ sufficiently small such that $(\mathcal{F}, \Delta+\epsilon C)$ is F-dlt and $\varphi$ is still a $\left(K_{\mathcal{F}}+\Delta+\epsilon C\right)$-flip (resp. $\left(K_{\mathcal{F}}+\Delta+\epsilon C\right)$ divisorial contraction). Thus, if $\mathcal{F}^{\prime}$ is the transformed foliation on $X^{\prime}$, then Lemma 3.11 implies that $\left(\mathcal{F}^{\prime}, \varphi_{*}(\Delta+\epsilon C)\right)$ is $F$-dlt. By Remark 3.7, we have that $\left(\mathcal{F}^{\prime}, \varphi_{*}(\Delta+\epsilon C)\right)$ is log canonical and, therefore, $\varphi_{*} C$ does not contain any lc centre of $\left(\mathcal{F}^{\prime}, \varphi_{*} \Delta\right)$.

Moreover, $\varphi_{*} A \sim_{\mathbb{Q}} \varphi_{*} C+H$. Thus, for a sufficiently small rational number $\delta>0$, we may choose $A^{\prime}=\delta H$ and $C^{\prime}=(1-\delta) \varphi_{*} A+\delta \varphi_{*} C$ and the claim follows. 


\subsection{F-dlt modification}

Definition 3.28 Let $\mathcal{F}$ be a co-rank one foliation on a normal projective variety $X$. Let $(\mathcal{F}, \Delta)$ be a foliated pair. A F-dlt modification for the foliated pair $(\mathcal{F}, \Delta)$ is a birational projective morphism $\pi: Y \rightarrow X$ such that if $\mathcal{G}$ is the pulled back foliation on $Y$ then $\left(\mathcal{G}, \pi_{*}^{-1} \Delta+\sum \epsilon\left(E_{i}\right) E_{i}\right)$ is F-dlt where we sum over all $\pi$-exceptional divisors and

$$
K_{\mathcal{G}}+\pi_{*}^{-1} \Delta+\sum \epsilon\left(E_{i}\right) E_{i}+F=\pi^{*}\left(K_{\mathcal{F}}+\Delta\right)
$$

for some $\pi$-exceptional $\mathbb{Q}$-divisor $F \geq 0$ on $Y$.

In particular, if $(\mathcal{F}, \Delta)$ is lc then $\pi$ only extracts divisors $E_{i}$ of discrepancy $-\epsilon\left(E_{i}\right)$.

Theorem 8.1 below implies the existence of a F-dlt modification for any foliated pair $(\mathcal{F}, \Delta)$ on a normal projective variety of dimension at most three.

\subsection{F-dlt cone and contraction theorem}

In [41] the cone theorem is proved under the hypotheses that $X$ is a $\mathbb{Q}$-factorial threefold, $\mathcal{F}$ is non-dicritical, $(\mathcal{F}, \Delta)$ has canonical singularities and the contraction theorem is proved under the additional hypothesis that $(\mathcal{F}, \Delta)$ is terminal at the generic points of Sing $X$.

In fact, it is possible to prove the cone and contraction theorem under the hypothesis that $(\mathcal{F}, \Delta)$ is F-dlt (rather than canonical). Even better, it is possible to prove the cone theorem in the case that $X$ is not necessarily $\mathbb{Q}$-factorial but $X$ is potentially klt. We still require that $\mathcal{F}$ has non-dicritical singularities. We explain the required modifications to the cone theorem first.

Since $X$ is potentially klt, Theorem 2.23 implies that there exists a small $\mathbb{Q}$-factorialisation $\pi: Y \rightarrow X$. Write $K_{\mathcal{G}}+\Gamma=\pi^{*}\left(K_{\mathcal{F}}+\Delta\right)$, where $\mathcal{G}$ is the pulled back foliation on $Y$.

Lemma 3.29 Set up as above. Then $(\mathcal{G}, \Gamma)$ is $F$-dlt.

Proof By Lemma 3.8, it follows that $(\mathcal{F}, \Delta)$ is foliated log smooth at the generic point of every lc centre of $(\mathcal{F}, \Delta)$. In particular, $\pi(\operatorname{Exc} \pi)$ does not contain any lc centre of $(\mathcal{F}, \Delta)$. Let $\rho: X^{\prime} \rightarrow X$ be a foliated log resolution of $(\mathcal{F}, \Delta)$ such that $a(E, \mathcal{F}, \Delta)>-\epsilon(E)$ for any $\rho$-exceptional divisor $E$. Let $p: \bar{Y} \rightarrow Y$ and $q: \bar{Y} \rightarrow X^{\prime}$ be proper birational morphisms which resolve the indeterminacy locus of the induced birational contraction $X^{\prime} \rightarrow Y$. Let $\overline{\mathcal{F}}$ be the transformed foliation on $\bar{Y}$ and let $\bar{\Delta}$ be the strict transform of $\Delta$ in $\bar{Y}$. Let $Y^{\prime} \rightarrow \bar{Y}$ be a foliated $\log$ resolution of $(\overline{\mathcal{F}}, \bar{\Delta}+G)$, where $G$ is the support of the exceptional divisor of $q$, and let $\rho^{\prime}: Y^{\prime} \rightarrow Y$ be 
the induced morphism. We may assume that the morphism $Y^{\prime} \rightarrow X^{\prime}$ is an isomorphism away from $\phi^{-1}(\operatorname{Exc} \pi)$. Suppose by contradiction that there exists a $\rho^{\prime}$-exceptional divisor $F$ such that $a(F, \mathcal{G}, \Gamma)=-\epsilon(F)$. Then the centre of $F$ in $Y$ does not intersect Exc $\pi$ and therefore the image of $F$ in $X^{\prime}$ is a divisor which is $\rho$-exceptional. Since $a(F, \mathcal{F}, \Delta)=a(F, \mathcal{G}, \Gamma)=-\epsilon(F)$, we get a contradiction and our claim follows.

Notice that $\left(K_{\mathcal{G}}+\Gamma\right) \cdot C=0$ for every $\pi$-exceptional curve $C$ so if $R$ is a $\left(K_{\mathcal{F}}+\Delta\right)$-negative extremal ray then there exists a $\left(K_{\mathcal{G}}+\Gamma\right)$-negative extremal ray $R^{\prime}$ such that $\pi_{*} R^{\prime}=R$. Thus, by replacing $(\mathcal{F}, \Delta)$ by $(\mathcal{G}, \Gamma)$ we may freely assume that $X$ is $\mathbb{Q}$-factorial.

Let $R$ be a $\left(K_{\mathcal{F}}+\Delta\right)$-negative extremal ray and let $H_{R}$ be a supporting hyperlane to $R$. We may assume that $H_{R}-\left(K_{\mathcal{F}}+\Delta\right)$ is ample and, in particular, $H_{R}^{3}>\left(K_{\mathcal{F}}+\Delta\right) \cdot H_{R}^{2}$.

If $H_{R}^{3}=0$ then [41, Corollary 2.28] implies that $X$ is covered by rational curves tangent to $\mathcal{F}$ which span $R$. Otherwise, if $H_{R}^{3}=0$ then $H_{R}$ is big and there exists an effective divisor $S$ such that $S$ is negative on $R$. As in the proof of [41, Lemma 4.7], it follows that if $(\mathcal{F}, \Delta)$ is $\log$ canonical then $R$ is spanned by a curve $C$. In either case, every $\left(K_{\mathcal{F}}+\Delta\right)$-negative extremal ray is spanned by a curve.

Lemma 3.30 Let $X$ be a normal threefold and let $\mathcal{F}$ be a co-rank one foliation with non-dicritical singularities on $X$. Suppose that $(\mathcal{F}, \Delta)$ is a log canonical foliated pair and that $X$ is potentially klt. Let $R$ be $a\left(K_{\mathcal{F}}+\Delta\right)$-negative extremal ray and suppose that $\operatorname{loc}(R) \neq X$. Suppose that $[C] \in R$.

Then $C$ is tangent to $\mathcal{F}$.

Proof This is proven in [41, Lemma 8.10] under the assumption that $X$ is $\mathbb{Q}$-factorial and klt. However, one can observe that the proof does not rely on either of these hypotheses.

For the reader's convenience, we briefly sketch the relevant ideas. Let $H_{R}$ be the supporting hyperplane to $R$ and suppose for sake of contradiction that $C$ is transverse to $\mathcal{F}$. Since $\operatorname{loc}(R) \neq X$, it follows that $H_{R}$ is big. Let $A$ be an ample $\mathbb{Q}$-Cartier divisor so that $H_{R}-A$ is still big. We may then find a $\mathbb{Q}$-divisor $0 \leq G \sim_{\mathbb{Q}} H_{R}-A$ and notice that $G \cdot C<0$.

Let $\lambda \geq 0$ be the largest real number so that $(\mathcal{F}, \Delta+\lambda G)$ is log canonical at the generic point of $C$. On one hand, we know that $\left(K_{\mathcal{F}}+\Delta+\lambda G\right) \cdot C<0$. On the other hand, by replacing $X$ by a small $\mathbb{Q}$-factorialisation (which is necessarily an isomorphism at the generic point of $C$ ) we may apply subadjunction (cf. [41, Theorem 4.5]) to see that since $C$ is transverse to $\mathcal{F}$ then $\left(K_{\mathcal{F}}+\Delta+\lambda G\right) \cdot C \geq 0$, a contradiction.

By Lemma 3.12, if $C$ is tangent to $\mathcal{F}$ then $(\mathcal{F}, \Delta)$ is canonical at the generic point of $C$ and we have reduced to the cone theorem in [41, Theorem 7.1]. 
Thus, we have:

Theorem 3.31 Let $X$ be a normal projective threefold and let $\mathcal{F}$ be a co-rank one foliation with non-dicritical singularities. Suppose that $X$ is potentially klt. Let $(\mathcal{F}, \Delta)$ be a $F$-dlt pair and let $H$ be an ample $\mathbb{Q}$-divisor.

Then there exist countably many curves $\xi_{1}, \xi_{2}, \ldots$ such that

$$
\overline{N E(X)}=\overline{N E(X)}_{K_{\mathcal{F}}+\Delta \geq 0}+\sum \mathbb{R}_{+}\left[\xi_{i}\right]
$$

Furthermore, for each $i, \xi_{i}$ is a rational curve tangent to $\mathcal{F}$ such that $\left(K_{\mathcal{F}}+\Delta\right) \cdot \xi_{i} \geq-6$, and if $C \subset X$ is a curve such that $[C] \in \mathbb{R}_{+}\left[\xi_{i}\right]$ and $\operatorname{loc}\left(\mathbb{R}_{+}\left[\xi_{i}\right]\right) \neq X$ then $C$ is tangent to $\mathcal{F}$. If we assume in addition that $X$ is $\mathbb{Q}$-factorial then if $[C] \in \mathbb{R}_{+}\left[\xi_{i}\right]$ then $C$ is tangent to $\mathcal{F}$.

In particular, there exist only finitely many $\left(K_{\mathcal{F}}+\Delta+H\right)$-negative extremal rays.

Remark 3.32 We will return to the problem of constructing contractions in the $\mathbb{Q}$-factorial case in Theorem 6.7 and in the non- $\mathbb{Q}$-factorial case in Sect. 8.1.

We now show that the MMP preserves non-dicritical singularities.

Lemma 3.33 Let $X$ be a normal threefold and let $\mathcal{F}$ be a non-dicritical corank one foliation on $X$. Suppose that $(\mathcal{F}, \Delta)$ is $\log$ canonical and that $X$ is potentially klt.

Let $R$ be a $\left(K_{\mathcal{F}}+\Delta\right)$-negative extremal ray and let $c: X \rightarrow Y$ be the contraction associated to $R$. Suppose that $c$ is a birational morphism and let $\mathcal{G}:=c_{*} \mathcal{F}$. Then $\mathcal{G}$ is non-dicritical. In particular, if $c$ is a flipping contraction and assuming that its flip $\phi: X \rightarrow X^{+}$exists, then $\mathcal{F}^{+}:=\phi_{*} \mathcal{F}$ is nondicritical.

Proof To prove our first claim let $q \in Y$ be a point and let $\pi: Y^{\prime} \rightarrow Y$ be any sequence of blow ups such that $\pi^{-1}(q)$ is a divisor. Perhaps passing to a higher model we may assume we have a factorisation $Y^{\prime} \stackrel{g}{\rightarrow} X \stackrel{c}{\rightarrow} Y$. Let $\mathcal{G}^{\prime}:=\pi_{*}^{-1} \mathcal{G}$. Lemma 3.30 implies that $c^{-1}(q)$ is tangent to $\mathcal{F}$. Since $g^{-1}\left(c^{-1}(q)\right)$ is a divisor and since $\mathcal{F}$ is non-dicritical it follows by definition of tangency that $g^{-1}\left(c^{-1}(q)\right)$ is an invariant divisor, as required.

We now prove our second claim. Let $c^{+}: X^{+} \rightarrow Y$ be the induced morphism. We have that $c_{*}^{+} \mathcal{F}=\mathcal{G}$ and, by our first claim, it is non-dicritical. This implies that $\mathcal{F}^{+}$is non-dicritical, as claimed.

Note that, in Sect. 6, we prove the existence of flips for non-dicritical F-dlt pairs defined on a $\mathbb{Q}$-factorial projective theefold. 


\section{Approximating formal divisors}

One of the main difficulties to prove the existence of flips for foliated pairs $(\mathcal{F}, \Delta)$, as in Theorem 6.4, is due to the fact that in the singular setting, some of the separatrices through a curve $C$ which is tangent to $\mathcal{F}$, are defined only in a formal neighbourhood of the curve $C$. To this end, since the MMP for formal schemes is still unknown, we study some application of Artin and Elkik's approximation theorems.

We begin by recalling some definitions. The following definition is [37, Chapter XI, Définition 2].

Definition 4.1 Let $A$ be a ring, $\mathfrak{J} \subset A$ an ideal and $B$ an étale $A$-algebra. We say that $B$ is an étale neighborhood of $\mathfrak{J}$ in $A$ if the morphism

$$
A / \mathfrak{J} A \rightarrow B / \mathfrak{J} B
$$

is an isomorphism.

The following definition is [37, Chapter XI, Définition 3].

Definition 4.2 A pair $(A, \mathfrak{J})$ of a ring and an ideal is called a henselian couple if $\mathfrak{J}$ is contained in the Jacobson radical of $A$ and for all étale neighborhoods $B$ of $\mathfrak{J}$ in $A$, there exists an $A$-morphism $B \rightarrow A$.

Given a pair $(A, \mathfrak{J})$ of a ring $A$ and an ideal $\mathfrak{J}$ contained in the Jacobson radical of $A$, it is possible to define the henselization of $(A, \mathfrak{J})$ as in $[37$, Chapitre XI, Théorème 2].

The next result is [17, Theorem 3 (see also the paragraph below Theorem 3 )]:

Theorem 4.3 (Elkik approximation) Let $(A, \mathfrak{J})$ be a henselian couple where $A$ is Noetherian and let $\widehat{A}$ be the $\mathfrak{J}$-adic completion. Let $\bar{M}$ be a finite type $\widehat{A}$-module, locally free on Spec $\widehat{A} \backslash V(\mathfrak{J})$.

Then there exists an A-module $M$ such that $M \otimes_{A} \widehat{A}$ is isomorphic to $\bar{M}$.

Furthermore, for any positive integer $k$, we may choose $M$ so that if

$$
\widehat{A}^{p} \stackrel{\bar{L}}{\rightarrow} \widehat{A}^{q} \rightarrow \bar{M} \rightarrow 0
$$

is a presentation of $\bar{M}$, then we have a presentation of $M$

$$
A^{p} \stackrel{L}{\rightarrow} A^{q} \rightarrow M \rightarrow 0
$$

such that $L=\bar{L} \bmod \mathfrak{J}^{k}$ and such that the isomorphism between $\bar{M}$ and $M \otimes \widehat{A}$ is induced by automorphisms of $\widehat{A}^{p}$ and $\widehat{A}^{q}$ congruent to the identity modulo $\mathfrak{J}^{k}$. 
Remark 4.4 Note that, in the Theorem above, after possibly replacing $M$ by $M^{* *}$, we may assume that $M$ is reflexive. Indeed, by [24, Proposition 1.8] it follows that $M^{* *} \otimes_{A} \widehat{A}$ is reflexive and, therefore, it coincides with $\bar{M}$.

Corollary 4.5 Let $(A, \mathfrak{J})$ be a henselian couple where $A$ is Noetherian and let $\widehat{A}$ be the $\mathfrak{J}$-adic completion. Suppose moreover that A is excellent and normal.

Fix any positive integer $k$. Let $\bar{V} \subset$ Spec $\widehat{A}$ be an effective integral divisor and let $m$ be a positive integer such that $m \bar{V}$ is Cartier away from $W=V(\mathfrak{J})$.

Then there exists a divisor $V$ on Spec $A$ such that

1. $\mathcal{O}(m V) \otimes \widehat{A} \cong \mathcal{O}(m \bar{V})$,

2. $V=\bar{V} \bmod \mathfrak{J}^{k}$.

Proof We first assume that $\bar{M}:=\mathcal{O}(\bar{V})$ is locally free away from $W$. Note that this case follows from [17, Corollaire p. 574], but we include a proof for the reader's convenience.

By Theorem 4.3 and Remark 4.4, there exists a reflexive sheaf $M$ on Spec $A$ such that $M \otimes \widehat{A}=\mathcal{O}(\bar{V})$. Let $\bar{s} \in \mathcal{O}(\bar{V})$ be a section whose associated divisor is $\bar{V}$. We may assume that there is a presentation

$$
\widehat{A}^{p} \stackrel{\bar{L}}{\rightarrow} \widehat{A}^{q} \rightarrow \bar{M} \rightarrow 0
$$

such that $\bar{s}$ is the image of $(1,0, \ldots, 0)$ in $\bar{M}$. Approximating this presentation by

$$
A^{p} \stackrel{L}{\rightarrow} A^{q} \rightarrow M \rightarrow 0
$$

we define $s$ to be the image of $(1,0, \ldots, 0)$ in $M$. In particular, we see that $s=\bar{s} \bmod \mathfrak{J}^{k}$ considered as sections of $\bar{M}$. Let $V$ be the divisor associated to $s$. Then $M=\mathcal{O}(V)$ and $V=\bar{V} \bmod \mathfrak{J}^{k}$.

Now, suppose that $m>1$ is the Cartier index of $\mathcal{O}(\bar{V})$ away from $W$. By Lemma 4.6, we may find a Galois possibly ramified cover $\sigma$ : Spec $B \rightarrow$ Spec $A$ such that $\left(\sigma^{*} \mathcal{O}(\bar{V})\right)^{* *}$ is a line bundle away from $\sigma^{-1}(W)$.

Note that $\mathfrak{J}^{\prime}=B \otimes \mathfrak{J}$ is the ideal corresponding to $\sigma^{-1}(W)$. Let $\widehat{B}$ be the $\mathfrak{J}^{\prime}$-adic completion of $B$. Observe that by [37, Chapter XI, Proposition 2] $\left(B, \mathfrak{J}^{\prime}\right)$ is a henselian couple. We may find a reflexive sheaf $M^{\prime}$ on Spec $B$ such that $M^{\prime} \otimes \widehat{B} \cong\left(\sigma^{*} \mathcal{O}(\bar{V})\right)^{* *}$. Let $\bar{s}$ be as above and let $\bar{t}=\sigma^{*} \bar{s}$.

As before, for any positive integer $\ell$, we can approximate $\bar{t}$ by a section $t$ of $M^{\prime}$ such that $t=\bar{t} \bmod \mathfrak{J}^{\prime \ell}$. Observe that for $\ell$ sufficiently large we have that $\sum_{g \in G} g \cdot t \neq 0$. So replacing $t$ by $\frac{1}{\# G} \sum_{g \in G} g \cdot t$ we may assume that $t$ is also $G$-invariant. Let $V^{\prime}$ be the divisor associated to $t$ and so $M^{\prime}=\mathcal{O}\left(V^{\prime}\right)$.

By Theorem 4.3 let $L$ be a reflexive sheaf on Spec $A$ such that $L \otimes \widehat{A}=$ $\mathcal{O}(m \bar{V})$. Then we have $\left(\sigma^{*} L\right)^{* *} \cong \mathcal{O}\left(m V^{\prime}\right)$. Thus $\left(\sigma^{*} L\right)^{* *}$ has a $G$-invariant section $t^{\otimes m}$ which descends to a section $\eta$ of $L$. 
If we set $V=\sigma\left(V^{\prime}\right)$, then notice that $(\eta=0)=m V$ and $\operatorname{so} \mathcal{O}(m V) \otimes \widehat{A} \cong$ $\mathcal{O}(m \bar{V})$. Next, we know that $m V=m \bar{V} \bmod \mathfrak{J}^{\ell}$ which implies that $V=\bar{V}$ $\bmod \mathfrak{J}^{\lfloor\ell / m\rfloor}$. Choosing $\ell \geq k m$ gives our result.

Lemma 4.6 Let $(A, \mathfrak{J})$ be a henselian couple where $A$ is Noetherian and let $\widehat{A}$ be the $\mathfrak{J}$-adic completion. Suppose moreover that $A$ is excellent.

Let $\bar{V} \subset$ Spec $\widehat{A}$ be a divisor such that $\mathcal{O}_{\text {Spec }} \widehat{A}(\bar{V})$ is $\mathbb{Q}$-Cartier away from $W=V(\mathfrak{J})$.

Then there exists a finite Galois morphism $\sigma: \operatorname{Spec} B \rightarrow \operatorname{Spec} A$ such that if $\widehat{\sigma}:$ Spec $\widehat{B} \rightarrow$ Spec $\widehat{A}$ is the completion of this map we have that $\left(\widehat{\sigma}^{*} \mathcal{O}(\bar{V})\right)^{* *}$ is locally free away from $\widehat{\sigma}^{-1}(W)$.

Proof Let $X=\operatorname{Spec} A$ and let $m$ be the Cartier index of $\mathcal{O}(\bar{V})$ away from $W$. Let $L$ be a reflexive sheaf on $X$ such that $L \otimes \widehat{A} \cong \mathcal{O}(m \bar{V})$ and whose existence is guaranteed by Theorem 4.3.

Let $\left\{U_{i}\right\}_{i=1, \ldots, n}$ be an open cover of Spec $A \backslash W$ and let $s_{i}$ be a global section of $L$ such that $\left.s_{i}\right|_{U_{i}}$ generates $\left.L\right|_{U_{i}}$. Choose a rational function $\varphi_{i}$ such that $\left(\varphi_{i}\right)=\left(\left.s_{i}\right|_{U_{i}}\right)$. Let $V_{i} \rightarrow U_{i}$ be a cover extracting a $m$-th root of $\varphi_{i}$. If we let $U_{i}=U_{i} \times{ }_{X}$ Spec $\widehat{A}$ and $\widehat{V}_{i}=V_{i} \times{ }_{X}$ Spec $\widehat{A}$ then we see that $\left.\mathcal{O}(\bar{V})\right|_{\widehat{V}_{i}} ^{* *}$ is locally free.

Let $V=V_{1} \times_{X} V_{2} \times_{X} \ldots \times_{X} V_{n}$ and let $K(V)$ be the field of functions of $V$ and notice that $K(V)$ is a finite extension of $K(A)$. Let $B$ be the integral closure of $A$ in $K(V)$. Note that $B$ is finite over $A$ (e.g. see [4, Proposition 5.17]). Thus, the natural map $\sigma: \operatorname{Spec} B \rightarrow \operatorname{Spec} A$ gives a cover with the desired properties.

Definition 4.7 Let $(A, \mathfrak{J})$ be a pair of a normal ring and an ideal. Let $\Delta^{\prime}$ be a $\mathbb{Q}$-divisor on Spec $A$. We say that a $\mathbb{Q}$-divisor $\Delta$ on $\operatorname{Spec} A$ approximates $\Delta^{\prime} \bmod \mathfrak{J}^{n}$ if we can write $\Delta=\sum a_{i} D_{i}$ and $\Delta^{\prime}=\sum a_{i} D_{i}^{\prime}$ where $D_{i}=D_{i}^{\prime}$ $\bmod \mathfrak{J}^{n}$.

Lemma 4.8 Let $(A, \mathfrak{J})$ be a pair of a ring and an ideal, where $A$ is excellent. Suppose that (Spec $A, \Delta)$ is a klt (resp. lc) pair.

Then there exists a positive integer $n_{0}$ such that if $n \geq n_{0}$ and if $\Delta^{\prime}$ is an approximation of $\Delta \bmod \mathfrak{J}^{n}$ such that $K_{\text {Spec } A}+\Delta^{\prime}$ is $\mathbb{Q}$-Cartier then (Spec $\left.A, \Delta^{\prime}\right)$ is klt (resp. lc).

Proof Let $\pi: Y \rightarrow \operatorname{Spec} A$ be a $\log$ resolution of (Spec $A, \Delta)$ and let $E=$ $\pi^{-1}(V(\mathfrak{J}))$ whose existence is guaranteed by [42, Theorems 1.1 .9 and 1.1.13]. Note that $\pi$ is projective. Perhaps passing to a higher resolution we may assume that $E$ is a divisor.

Let $\widehat{D}$ be a component of $\Delta$. We may write

$$
\pi^{-1} I_{\widehat{D}} \cdot \mathcal{O}_{Y}=\mathcal{O}_{Y}\left(-\widehat{D}^{\prime}-\sum a_{i} E_{i}\right)
$$


where $\widehat{D}^{\prime}$ is the strict transform of $\widehat{D}$ and $E_{i}$ are $\pi$-exceptional and $a_{i} \geq 0$. Choose $r$ larger than $a_{i}$ for all $i$ and for all the components $\widehat{D}$ of $\Delta$.

Next, pick $n_{0}$ so that $\mathfrak{J}^{n_{0}} \subset \pi_{*} \mathcal{O}_{Y}(-2 r E)$. Note that in this case $\pi^{-1} \mathfrak{J}^{n_{0}} \subset$ $\mathcal{O}_{Y}(-2 r E)$ and so if $s=t \bmod \mathfrak{J}^{n_{0}}$ then

$$
\pi^{*} s=\pi^{*} t \quad \bmod \mathcal{O}_{Y}(-2 r E) .
$$

This choice of $r, n_{0}$ guarantees that if $\widehat{D}$ is a component of Supp $\Delta$ and $D$ is an approximation of $\widehat{D} \bmod \mathfrak{J}^{n}$ where $n \geq n_{0}$ that if we write

$$
\pi^{-1} I_{\widehat{D}} \cdot \mathcal{O}_{Y}=\mathcal{O}_{Y}\left(-\widehat{D}^{\prime}-\sum a_{i} E_{i}\right)
$$

and

$$
\pi^{-1} I_{D} \cdot \mathcal{O}_{Y}=\mathcal{O}_{Y}\left(-D^{\prime}-\sum b_{i} E_{i}\right)
$$

then $a_{i}=b_{i}$ and so $D^{\prime}=\widehat{D}^{\prime}$ in some infinitessimal neighborhood of $E$. Thus, if $\Delta^{\prime}$ is an approximation of $\Delta \bmod \mathfrak{J}^{n}$ for $n \geq n_{0}$ then $\pi$ is also a log resolution of ( $\left.\operatorname{Spec} A, \Delta^{\prime}\right)$.

Furthermore, if we let $\pi_{*}^{-1} \Delta$ (respectively $\pi_{*}^{-1} \Delta^{\prime}$ ) be the strict transform of $\Delta$ (respectively $\Delta^{\prime}$ ) we see that $\left.\pi_{*}^{-1} \Delta\right|_{E}=\left.\pi_{*}^{-1} \Delta^{\prime}\right|_{E}$ which implies that the two are $\pi$-numerically equivalent and hence the discrepancies of (Spec $A, \Delta$ ) and $\left(\operatorname{Spec} A, \Delta^{\prime}\right)$ are the same and the result follows.

\section{Approximating formal separatrices}

In this section we work in the following set up:

Let $X$ be a $\mathbb{Q}$-factorial and klt quasi-projective threefold. Let $\mathcal{F}$ be a co-rank one foliation on $X$ with non-dicritical singularities and let $(\mathcal{F}, \Delta)$ be a F-dlt foliated pair. Let $f: X \rightarrow Z$ be a birational contraction and $p \in f(\operatorname{Exc} f)$ be a closed point. We assume that $D:=f^{-1}(p)$ is tangent to $\mathcal{F}$. Suppose moreover that $Z$ is klt away from finitely many points. As we will see, this will always be the case for birational contractions arising in the course of the MMP.

Let $\hat{f}: \widehat{X} \rightarrow \widehat{Z}$ be the completion of $f$ along the fibre $f^{-1}(p)$, and let $\widehat{\mathcal{F}}$ be the formal foliation.

Remark 5.1 Observe that by Lemmas 3.12 and 3.14 if $D$ is a curve and $D_{i}$ is component of $D$ then $D_{i}$ is contained in a (possibly formal) $\widehat{\mathcal{F}}$-invariant divisor.

Lemma 5.2 Let $\widehat{S} \subset \widehat{X}$ be any irreducible formal divisor not contained in Exc $f$. Fix an integer $n>0$. 
Then there exists an étale morphism $\sigma: Z^{\prime} \rightarrow Z$ and a divisor $S^{\prime}$ on $X^{\prime}=$ $X \times_{Z} Z^{\prime}$ such that if $\tau: X^{\prime} \rightarrow X$ is the projection, $f^{\prime}: X^{\prime} \rightarrow Z^{\prime}$ the induced morphism, $D^{\prime}=f^{\prime-1}\left(\sigma^{-1}(p)\right)$ and $\widehat{\tau}: \widehat{X}^{\prime} \rightarrow \widehat{X}$ is the completion of $\tau$ along $D^{\prime}$, then $\widehat{S}^{\prime}=\widehat{\tau}^{*} \widehat{S} \bmod I_{D^{\prime}}^{n}$, where $\widehat{S}^{\prime}$ is the restriction of $S^{\prime}$ to $\widehat{X}^{\prime}$.

Proof We may assume that $Z=\operatorname{Spec} B$ is affine. Let $(A, \mathfrak{m})$ be the henselisation of $B$ at $p$, and let $\widehat{A}$ be the formal completion of $B$ at $p$. Let $\widetilde{X}=X \times$ Spec $B$ Spec $\widehat{A}$. By the Grothendieck existence theorem there exists a divisor $\widetilde{S}$ on $\widetilde{X}$ such that $\left.\widetilde{S}\right|_{\widehat{X}}=\widehat{S}$.

Let $\widetilde{f}: \widetilde{X} \rightarrow$ Spec $\widehat{A}$ be the induced morphism. By the proper mapping theorem, we have that $\bar{V}:=\widetilde{f}_{*} \widetilde{S}$ is a divisor on Spec $\widehat{A}$. We claim that $\bar{V}$ is a $\mathbb{Q}$ Cartier divisor on Spec $\widehat{A} \backslash p$. Indeed, by assumption $Z$ is klt away from finitely many points. By [21, Proposition 9.4] a klt variety has quotient singularities in codimension two, and so it follows that, away from finitely many points, $Z$ has quotient singularities. In particular, it follows that Spec $\widehat{A} \backslash\{p\}$ is $\mathbb{Q}$-factorial and so $\bar{V}$ is $\mathbb{Q}$-Cartier.

Let $\widetilde{D}=\widetilde{f}^{-1}(p)$. Pick a positive integer $k$ large enough so that $\mathfrak{m}^{k} \subset$ $\widetilde{f}_{*} I_{\widetilde{D}}^{n+n^{\prime}}$ where $n^{\prime}$ is a sufficiently large positive integer so that $\mathcal{O}(-\bar{V})$ is not contained in $\widetilde{f}_{*} I_{\widetilde{D}}^{n^{\prime}}$. By Corollary 4.5, there exists a divisor $V$ on Spec $A$ which agrees with $\bar{V} \bmod \mathfrak{m}^{k}$. Recall

$$
A=\underset{\left(\operatorname{Spec} B^{\prime}, q\right) \rightarrow(\operatorname{Spec} B, p)}{\lim _{\longrightarrow}} B^{\prime}
$$

where we run over étale morphisms ( $\left.\operatorname{Spec} B^{\prime}, q\right) \rightarrow(\operatorname{Spec} B, p)$ sending $q$ to $p$. Thus, we see that there exists some étale cover $\operatorname{Spec} B^{\prime} \rightarrow \operatorname{Spec} B$ and a divisor $V^{\prime}$ on Spec $B^{\prime}$ which agrees with $V$ when pulled back to Spec $A$.

Let $S^{\prime}$ be the strict transform of $V^{\prime}$ on $X^{\prime}$ and let $\widehat{S^{\prime}}$ be the restriction of $S^{\prime}$ to $\widehat{X}^{\prime}$. Then we have that $\widehat{S^{\prime}}=\hat{\tau}^{*} \widehat{S} \bmod I_{D^{\prime}}^{n}$, as required.

Lemma 5.3 Notation as above. Let $\widehat{S}_{1}, \ldots, \widehat{S}_{k}$ be any collection of distinct irreducible $\widehat{\mathcal{F}}$-invariant divisors and suppose that $\widehat{S}_{1}, \ldots, \widehat{S}_{k}$ are $\mathbb{Q}$-Cartier and such that $\widehat{S}_{i}$ is not contained in Exc $f$ for any $i$. Let $X^{\prime}$ and $S_{1}^{\prime}, \ldots, S_{k}^{\prime}$ be as in Lemma 5.2, where $S_{i}^{\prime}$ is an approximation of $\widehat{S}_{i} \bmod I_{D^{\prime}}^{n}$.

Then for $n$ large enough and perhaps shrinking $X^{\prime}$ to a smaller neighborhood of $D^{\prime}$ we have $\left(X^{\prime}, \sum S_{i}^{\prime}\right)$ is log canonical.

Proof We first claim that $S_{i}^{\prime}$ is $\mathbb{Q}$-Cartier. Indeed, by construction we have an isomorphism $\hat{f}_{*} \mathcal{O}_{\widehat{X}}\left(\widehat{S}_{i}\right) \cong \hat{f}_{*} \mathcal{O}_{\widehat{X}}\left(S_{i}^{\prime}\right)$ of reflexive sheaves on $\widehat{Z}$. Since $\mathcal{O}_{\widehat{X}}\left(\widehat{S}_{i}\right)$ and $\mathcal{O}_{\widehat{X}}\left(S_{i}^{\prime}\right)$ are reflexive and $\widehat{S}_{i}$ and $S_{i}^{\prime}$ do not contain any $f$-exceptional divisors in their support it follows that this isomorphism on $Z$ gives an isomorphism $\mathcal{O}_{\widehat{X}}\left(\widehat{S}_{i}\right) \cong \mathcal{O}_{\widehat{X}}\left(S_{i}^{\prime}\right)$ on $\widehat{X}$. In particular, $S_{i}^{\prime}$ is $\mathbb{Q}$-Cartier because $\widehat{S}_{i}$ is. 
The result then follows by combining Lemmas 4.8 and 3.16.

\section{Constructing the flip}

\subsection{Set up}

Through out this section, we assume that $X$ is a $\mathbb{Q}$-factorial klt quasi-projective threefold, $\mathcal{F}$ is a co-rank one foliation on $X$ with non-dicritical singularities and $(\mathcal{F}, \Delta)$ is $\mathrm{F}$-dlt foliated pair.

The goal of this section is to show that if $f: X \rightarrow Z$ is a flipping contraction associated to a $\left(K_{\mathcal{F}}+\Delta\right)$-negative extremal ray $R$, then the $\left(K_{\mathcal{F}}+\Delta\right)$-flip exists (cf. Sect. 2.6). The basic idea is to reduce the $\left(K_{\mathcal{F}}+\Delta\right)$-flip to a $\left(K_{X}+\widetilde{\Delta}\right)$-flip for some klt pair $(X, \widetilde{\Delta})$.

Recall that in [41, Lemma 8.21] it was proven that the flipping contraction exists in the category of algebraic spaces. Observe that the proof given there only requires that $(\mathcal{F}, \Delta)$ has non-dicritical and $\log$ canonical singularities and so works for F-dlt foliated pairs with non-dicritical singularities.

Our goal here is to show that

(a) if $X$ is projective then also $Z$ is projective and $\rho(X / Y)=1$.

(b) the flip exists.

By Remark 3.7 and Lemma 3.30, it follows that Exc $f$ is tangent to the foliation.

The following result will be needed to address (a) and (b) above:

Lemma 6.1 Without loss of generality, we may assume that $\lfloor\Delta\rfloor=0$.

Proof The $\left(K_{\mathcal{F}}+\Delta\right)$-flip is the $\left(K_{\mathcal{F}}+(1-\epsilon) \Delta\right)$-flip for $\epsilon>0$ sufficiently small.

The following result will be needed to address (b) above:

Lemma 6.2 Let $f: X \rightarrow Y$ be a small contraction between algebraic spaces. Let $D$ be a $\mathbb{Q}$-Cartier divisor on $X$ such that $-D$ is $f$-ample. Let $\left\{U_{i} \rightarrow Y\right\}_{i \in I}$ be an étale cover of $Y$. Suppose that, for each $X_{i}=X \times_{Y} U_{i} \rightarrow U_{i}$, the $\left.D\right|_{X_{i}}{ }^{-}$ flip exists.

Then the D-flip exists.

Proof The existence of the flip is equivalent to the $\mathcal{O}_{Y}$-algebra

$$
\bigoplus_{m \geq 0} f_{*} \mathcal{O}_{X}(m D)
$$

being finitely generated, where $m$ is taken to be sufficiently divisble. Finite generation of an algebra can be checked étale locally and the result follows. 
We briefly sketch the remainder of the goals for this subsection. First, we show that, after possibly replacing $X$ by an appropriate small modification, we may find a formal divisor $\widehat{F}$ on $\widehat{X}$, the formal completion of $X$ along Exc $f$, such that $K_{\widehat{X}}+\widehat{F}$ is numerically equivalent to $K_{\widehat{\mathcal{F}}}$ and such that $(\widehat{X}, \widehat{F})$ has $\log$ canonical singularities. Indeed, we will show that if $\widehat{S}$ is the sum of all the $\mathcal{F}$-invariant divisors meeting Exc $f$ then $K_{\widehat{\mathcal{F}}}-\left(K_{\widehat{X}}+\widehat{S}\right)$ is $f$-nef. Since Exc $f$ is a curve, it is then easy to find an effective divisor $D$ on some analytic neighborhood of Exc $f$ such that $D$ restricted to $\widehat{X}$ is numerically equivalent to $K_{\widehat{\mathcal{F}}}-\left(K_{\widehat{X}}+\widehat{S}\right)$. Then $\widehat{F}=\widehat{S}+D$ will be our desired divisor. Second, we will apply our version of Elkik approximation theorem to show that we may approximate $\widehat{F}$ on some étale neighborhood $X^{\prime} \rightarrow X$ of Exc $f$ by a divisor $F^{\prime}$. We will then, in the next subsection, construct the foliated flip as a $\left(K_{X^{\prime}}+F^{\prime}\right)$-flip.

We now proceed with the construction outlined above. Let $S_{1}, \ldots, S_{\ell}$ be the collection of all the $\mathcal{F}$-invariant divisors (formal or otherwise) on $X$ meeting at least one of the curves contracted by $f$, whose existence is guaranteed by Remark 5.1. We remark that by Lemma 3.5 we may extend each $S_{i}$ to a formal divisor on $\widehat{X}$. We emphasise that each $f$-exceptional curve is contained in one such divisor. By Lemma 5.2, we may find a diagram

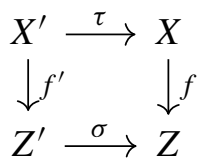

where $\sigma: Z^{\prime} \rightarrow Z$ is étale and divisors $S_{k}^{\prime}$ on $X^{\prime}$ which approximate the $S_{k}$ to some arbitrarily high (but fixed) order. Let $\mathcal{F}^{\prime}$ be the transformed foliation on $X^{\prime}$.

Note that $X^{\prime}$ is klt since $X$ is. Let $g: Y \rightarrow X^{\prime}$ be a small $\mathbb{Q}$-factorialisation of $X^{\prime}$ and let $\mathcal{F}_{Y}$ the pulled back foliation on $Y$. Then $g^{*} K_{\mathcal{F}^{\prime}}=K_{\mathcal{F}_{Y}}$ and $g^{*} K_{X^{\prime}}=K_{Y}$. Let $h=f^{\prime} \circ g: Y \rightarrow Z^{\prime}$ be the composition and write Exc $h=$ $C=\cup C_{i}$.

Let $U$ be a small analytic neighbourhood around $C$. We claim that, for each $i$, we can find $\mathbb{Q}$-divisors $D_{i 1}, \ldots, D_{i m_{i}} \geq 0$ on $U$ such that $D_{i k} \cdot C_{j}=\delta_{i j}$ for each $k=1, \ldots, m_{i}$, where $m_{i} \geq 0$ is an integer whose precise value will be determined later. Indeed, let $A$ be a general sufficiently ample Cartier divisor on $Y$ so that $A \cap C=\left\{P_{i j}\right\}$ is a finite collection of points where $P_{i j} \in C_{i}$ and $P_{i j} \notin C_{k}$ for $k \neq i$. Then, on a sufficiently small analytic neighborhood $U$ of $C$ we may write $A \cap U=\sum A_{i j}$ where $A_{i j} \cap C=P_{i j}$ and $A_{i j} \cap A_{i^{\prime} j^{\prime}}=\emptyset$ for $(i, j) \neq\left(i^{\prime}, j^{\prime}\right)$. Taking $D_{i 1}, \ldots, D_{i m_{i}}$ to be some of the $A_{i j}$ as $A$ varies across ample divisors will then have our desired properties 
We remark that if we write $g^{*}\left(K_{\mathcal{F}^{\prime}}+\Delta^{\prime}\right)=K_{\mathcal{F}_{Y}}+\Delta_{Y}$ then by Lemma 3.29, we have that $\left(\mathcal{F}_{Y}, \Delta_{Y}\right)$ is F-dlt. Moreover, since $\mathcal{F}^{\prime}$ has non-dicritical singularities it follows that each $g$-exceptional curve is tangent to $\mathcal{F}_{Y}$.

Let $\widetilde{S}_{k}$ be the strict transform of $S_{k}^{\prime}$ on $Y$ and note that $\widetilde{S}_{k}$ is a $\mathbb{Q}$-Cartier divisor. Let $\widehat{Y}$ be the formal completion of $Y$ along $\operatorname{Exc} h$, and notice that we have a morphism $\hat{g}: \widehat{Y} \rightarrow \widehat{X}$. Let $T_{k}$ be the strict transform of $S_{k}$ on $\widehat{Y}$. We claim that $T_{k}$ is a $\mathbb{Q}$-Cartier divisor. Observe that we have an isomorphism of sheaves $\mathcal{O}_{\widehat{Y}}\left(T_{k}\right) \cong \mathcal{O}_{\widehat{Y}}\left(\widetilde{S}_{k}\right)$ : indeed, by item (1) of Corollary 4.5 we know that if $\hat{h}$ is the restriction of $h$ to $\widehat{Y}$ then $\hat{h}_{*} \mathcal{O}_{\widehat{Y}}\left(T_{k}\right) \cong \hat{h}_{*} \mathcal{O}_{\widehat{Y}}\left(\widetilde{S}_{k}\right)$ and since Exc $h$ is of codimension $\geq 2$ this gives an isomorphism of reflexive sheaves on $\widehat{Y}$. Since $\widetilde{S}_{k}$ is $\mathbb{Q}$-Cartier this implies that $T_{k}$ is $\mathbb{Q}$-Cartier as well.

For any $T_{j}$ which is convergent and contains one of the $C_{i}$ by Corollary 3.20 we may write $\left.\left(K_{\widehat{Y}}+\sum T_{k}\right)\right|_{T_{j}}=K_{T_{j}}+\Theta_{j}$ and $\left.K_{\mathcal{F}_{Y}}\right|_{T_{j}}=K_{T_{j}}+\Delta_{j}$ where $\Theta_{j} \geq \Delta_{j}$.

Now fix $i$ and consider $C_{i}$. We consider two possibilities as in Lemma 3.14. First, suppose that $\mathcal{F}_{Y}$ is terminal at the generic point of $C_{i}$. In this case there exists a single $k_{i}$ such that $C_{i} \subset T_{k_{i}}$ and moreover $T_{k_{i}}$ is convergent. Again, by Corollary 3.20 we know that $\Theta_{k_{i}}$ and $\Delta_{k_{i}}$ have the same coefficient along $C_{i}$.

Now suppose that $\mathcal{F}_{Y}$ is canonical (but not terminal) at the generic point of $C_{i}$. In particular, Lemma 3.8 implies that $\mathcal{F}_{Y}$ has simple singularities at a general point of $C_{i}$. Let $q \in C_{i}$ be a general point and let $S$ be the strong separatrix of $\mathcal{F}_{Y}$ at $q$, recall that $S$ is convergent. Then by Lemma 3.5 this separatrix may be extended to a convergent divisor containing $C_{i}$, which is therefore one of the $T_{k}$. Let $T_{k_{i}}$ be this divisor. Again, we may apply Corollary 3.20 to see that $\Theta_{k_{i}}$ and $\Delta_{k_{i}}$ have the same coefficient along $C_{i}$.

It follows that

$$
\left(K_{\widehat{Y}}+\sum T_{k}\right) \cdot C_{i} \leq K_{\mathcal{F}_{Y}} \cdot C_{i},
$$

and so

$$
\left(K_{Y}+\sum \widetilde{S}_{k}\right) \cdot C_{i} \leq K_{\mathcal{F}_{Y}} \cdot C_{i} .
$$

By Lemma 5.2, we may find a diagram

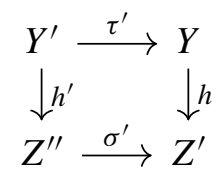

where $\sigma^{\prime}: Z^{\prime \prime} \rightarrow Z^{\prime}$ is étale and divisors $D_{i k}^{\prime}$ on $Y^{\prime}$ such that $D_{i k}^{\prime}$ approximate $D_{i k}$ to some arbitrarily high (but fixed) order. Notice that, as in the proof of 
the fact that $T_{k}$ is $\mathbb{Q}$-Cartier, we have that $D_{i k}^{\prime}$ is $\mathbb{Q}$-Cartier. Observe also that if $C_{i}^{\prime} \subset Y^{\prime}$ is an $h^{\prime}$-exceptional curve such that $\tau^{\prime}\left(C_{i}^{\prime}\right)=C_{i}$ then we still have $C_{i}^{\prime} \cdot D_{j k}^{\prime}=\delta_{i j}$.

Notice that if $\widetilde{S}_{k}^{\prime}$ is a surface on $Y^{\prime}$ such that $\tau^{\prime}\left(\widetilde{S}_{k}^{\prime}\right)=\widetilde{S}_{k}$, then we still have the inequality

$$
\left(K_{Y^{\prime}}+\sum \widetilde{S}_{k}^{\prime}\right) \cdot C^{\prime} \leq K_{\mathcal{F}_{Y^{\prime}}} \cdot C^{\prime}
$$

for any $h^{\prime}$-exceptional curve $C^{\prime}$. Thus, if we take

$$
a_{i}=\left(K_{\mathcal{F}_{Y^{\prime}}}-\left(K_{Y^{\prime}}+\sum \widetilde{S}_{k}^{\prime}\right)\right) \cdot C_{i}^{\prime} \geq 0
$$

where $C_{i}^{\prime}$ is a curve such that $\tau^{\prime}\left(C_{i}^{\prime}\right)=C_{i}$, then we have that

$$
K_{Y^{\prime}}+\sum \widetilde{S}_{k}^{\prime}+\sum \frac{a_{i}}{m_{i}} D_{i k}^{\prime} \equiv_{h^{\prime}} K_{\mathcal{F}_{Y^{\prime}}}
$$

Let $\Delta^{\prime}=\tau^{\prime *} g^{*} \tau^{*} \Delta$. Notice that $\left(\mathcal{F}_{Y^{\prime}}, \Delta^{\prime}\right)$ is F-dlt and the $\widetilde{S}_{k}^{\prime}$ are smooth in codimension one, and so Lemmas 3.16 and 4.8 imply that (perhaps replacing $Z^{\prime \prime}$ be an open neighborhood of $\left.h^{\prime}\left(\left(\tau^{\prime}\right)^{-1}(C)\right)\right)\left(Y^{\prime}, \Delta^{\prime}+\sum S_{k}^{\prime}\right)$ is lc and $\left(Y^{\prime}, \Delta^{\prime}+(1-\epsilon) \sum S_{k}^{\prime}\right)$ is klt for $0<\epsilon \leq 1$. So by choosing the $D_{i k}$ general enough and $m_{i}$ large enough so that $a_{i} \leq m_{i}$ we may assume that if $A=$ $\sum \widetilde{S}_{k}^{\prime}+\sum \frac{a_{i}}{m_{i}} D_{i k}^{\prime}$, then $\left(Y^{\prime}, \Delta^{\prime}+A\right)$ is lc and $\left(Y^{\prime}, \Delta+(1-\epsilon) A\right)$ is klt.

Since $X \rightarrow Z$ contracts only a single extremal ray and $-\left(K_{\mathcal{F}}+\Delta\right)$ is relatively ample, there exists $\lambda \in \mathbb{Q}$ such that $\lambda\left(K_{\mathcal{F}}+\Delta\right) \equiv_{f}\left(K_{X}+\Delta\right)$. Since $g$ is small and $\tau$ and $\tau^{\prime}$ are étale, it follows that $\lambda\left(K_{\mathcal{F}_{Y^{\prime}}}+\Delta^{\prime}\right) \equiv_{h^{\prime}}\left(K_{Y^{\prime}}+\Delta^{\prime}\right)$, where $\mathcal{F}_{Y^{\prime}}$ is the transformed foliation on $Y^{\prime}$. Since $A \equiv_{h^{\prime}}\left(K_{\mathcal{F}_{Y^{\prime}}}+\Delta^{\prime}\right)-$ $\left(K_{Y^{\prime}}+\Delta^{\prime}\right)$, we have

$$
A \equiv{ }_{h^{\prime}} \mu\left(K_{\mathcal{F}_{Y^{\prime}}}+\Delta^{\prime}\right)
$$

for $\mu=1-\lambda$.

\subsection{Existence of the flip}

Below, we use the same notation as in the previous subsection.

Lemma 6.3 Set up as above.

Then the following hold:

1. $\rho(X / Z)=1$; and

2. if, in addition, $X$ is projective, then also $Z$ is projective. 
Proof Let $D$ be a $\mathbb{Q}$-Cartier divisor on $X$ which is $f$-numerically trivial. In order to show that $\rho(X / Z)=1$, it is enough to show that there exists a $\mathbb{Q}$ Cartier divisor $M$ on $Z$ such that $f^{*} M=D$. Indeed, since $f: X \rightarrow Z$ is a flipping contraction associated to an extremal ray $R$, the claim implies that the sequence

$$
0 \longrightarrow \operatorname{Pic}(Z) \otimes \mathbb{Q} \stackrel{f^{*}}{\longrightarrow} \operatorname{Pic}(X) \otimes \mathbb{Q} \longrightarrow \mathbb{Q} \longrightarrow 0
$$

is exact, where the second to last arrow is given by $D \mapsto D \cdot \xi$ where $\xi$ is a fixed curve such that $[\xi] \in R$.

Let $D^{\prime}=\tau^{\prime *} g^{*} \tau^{*} D$. Since the descent problem above is étale local, it suffices to show that $D^{\prime}=\left(h^{\prime}\right)^{*} M^{\prime}$ for some $\mathbb{Q}$-Cartier divisor on $Z^{\prime}$. The existence of $M^{\prime}$ follows by applying the classical relative base point free theorem to the pair $\left(Y^{\prime}, \Delta^{\prime}+(1-\epsilon) A\right)$, for some $0<\epsilon \ll 1$. Indeed, recall from Sect. 6.1 we have that $\left(Y^{\prime}, \Delta^{\prime}+(1-\epsilon) A\right)$ is klt. Thus, since

$$
D^{\prime}-\left(K_{Y^{\prime}}+\Delta^{\prime}+(1-\epsilon) A\right) \equiv_{h^{\prime}}-(1-\epsilon \mu)\left(K_{\mathcal{F}_{Y^{\prime}}}+\Delta^{\prime}\right)
$$

is $h^{\prime}$-big and nef for small $\epsilon$, we have that $D^{\prime}$ is $h^{\prime}$-semi-ample. Thus, by definition, there is some $n \gg 0$ and a Cartier divisor $L$ on $Z^{\prime}$ such that $\left(h^{\prime}\right)^{*} L=$ $n D^{\prime}$. Thus, we may choose $M^{\prime}=\frac{1}{n} L$ and (1) follows.

Assume now that $X$ is projective and that $D=H_{R}$ is a nef $\mathbb{Q}$-Cartier divisor on $X$ which defines a supporting hyperplane for $R$ in $\overline{N E}(X)$ and let $M$ be the induced $\mathbb{Q}$-Cartier divisor on $Z$. The existence of $H_{R}$ is a direct consequence of the Cone theorem, [41] or Theorem 3.31. Projectivity then follows by noting that, for any subvariety $V$ of $Z$ we have $M^{\operatorname{dim} V} \cdot V>0$. Indeed, $M$ is ample by the Nakai-Moishezon criterion for algebraic spaces, [26, Theorem 3.11], and so $Z$ is projective. Thus, (2) follows.

Theorem 6.4 Let $X$ be a $\mathbb{Q}$-factorial projective threefold and let $\mathcal{F}$ be a corank one foliation on $X$ with non-dicritical singularities. Suppose that $(\mathcal{F}, \Delta)$ is a F-dlt foliated pair. Let $f: X \rightarrow Z$ be a $\left(K_{\mathcal{F}}+\Delta\right)$-negative flipping contraction.

Then the $\left(K_{\mathcal{F}}+\Delta\right)$-flip $X \rightarrow X^{+}$exists. Moreover,

1. $X^{+}$is projective and $\mathbb{Q}$-factorial,

2. $\left(\mathcal{F}^{+}, \Delta^{+}\right)$has $F$-dlt singularities,

3. If, in addition, the foliated pair $(\mathcal{F}, \Delta)$ admits terminal (resp. canonical, resp. log terminal, resp. log canonical) singularities then so does $\left(\mathcal{F}^{+}, \Delta^{+}\right)$,

4. $\mathcal{F}^{+}$has non-dicritical singularities and

5. $X^{+}$is klt. 
Proof By Lemma 6.2 it suffices to construct the flip étale locally on the base. Thus, taking $Z^{\prime \prime}$ as in Sect. 6.1, we see that to construct the flip it suffices to produce an ample model for $K_{\mathcal{F}_{Y^{\prime}}}+\Delta^{\prime}$ over $Z^{\prime \prime}$, see Sect. 2.6.

However, we know that for $\epsilon>0$ sufficiently small $K_{Y^{\prime}}+\Delta^{\prime}+A, K_{Y^{\prime}}+$ $\Delta+(1-\epsilon) A$ and $K_{\mathcal{F}_{Y^{\prime}}}+\Delta^{\prime}$ all have the same ample model over $Z^{\prime \prime}$. As above, we have that $\left(Y^{\prime}, \Delta^{\prime}+(1-\epsilon) A\right)$ is klt and so the ample model over $Z^{\prime \prime}$ exists by [7, Theorem 1.2]. Call this model

$$
c: Y^{\prime} \rightarrow Y^{\prime+} \text {. }
$$

Since $h^{\prime}: Y^{\prime} \rightarrow Z^{\prime \prime}$ is small, we know that $c$ is small and so $\left(Y^{\prime+}, c_{*}\left(\Delta^{\prime}+\right.\right.$ $(1-\epsilon) A))$ is in fact klt. Projectivity and $\mathbb{Q}$-factoriality follow easily.

Our claims on the singularities of $\left(\mathcal{F}^{+}, \Delta^{+}\right)$are a direct consequence of Lemma 2.7.

Non-dicriticality of $\mathcal{F}^{+}$follows from the fact that $X$ is klt by Lemmas 3.16 and 3.33 .

Remark 6.5 If one is so inclined this can all be done in the analytic topology around the flipping curves. The relevant classical log MMP is known to exist by [34].

Remark 6.6 Since the construction of the flip is local on the base, if $f: X \rightarrow Z$ is a flipping contraction between quasi-projective varieties then the arguments above show that the flip $\phi: X \rightarrow X^{+}$still exists and enjoys all the properties of the flip listed in Theorem 6.4, with the exception of projectivity of $X^{+}$.

\section{$6.3\left(K_{\mathcal{F}}+\Delta\right)$-negative contractions are extremal}

Theorem 6.7 Let $X$ be a normal $\mathbb{Q}$-factorial projective threefold and let $\mathcal{F}$ be a co-rank one foliation on $X$ with non-dicritical singularities. Let $(\mathcal{F}, \Delta)$ be a $F$-dlt pair and let $R$ be a $\left(K_{\mathcal{F}}+\Delta\right)$-negative extremal ray.

Then the contraction associated to $R$

$$
\phi_{R}: X \rightarrow Y
$$

exists in the category of projective varieties and $\rho(X / Y)=1$. In particular, $\phi_{R}$ is extremal. Moreover, if $\phi_{R}$ is a divisorial contraction then $Y$ is $\mathbb{Q}$-factorial and klt.

Proof First, observe that, by Lemma 3.16, $X$ is klt.

If $\operatorname{loc}(R)=X$, then, as in [41, Theorem 8.13], it follows that $R$ is in fact $K_{X}$-negative and so the contraction exists.

If $\operatorname{loc}(R)=D$ a divisor and $D$ is transverse to the foliation, then as in [41, Lemma 8.15], it follows that $R$ is $\left(K_{X}+\Delta\right)$-negative. If $D$ is invariant then we 
claim that that $R$ is $\left(K_{X}+\Delta+D\right)$-negative. Indeed, $D$ is covered by curves $C_{t}$ which span $R$ and if $D^{v} \rightarrow D$ is the normalisation of $D$ and we write

$$
\left.\left(K_{\mathcal{F}}+\Delta\right)\right|_{D^{v}}=K_{D^{v}}+\Theta \quad \text { and }\left.\quad\left(K_{X}+\Delta+D\right)\right|_{D^{v}}=K_{D^{v}}+\Theta^{\prime}
$$

then Corollary 3.20 implies that $\left(\Theta-\Theta^{\prime}\right) \cdot C_{t} \geq 0$ and so since $\left(K_{D^{\nu}}+\Theta\right) \cdot C_{t}<$ 0 we see that $\left(K_{D^{v}}+\Theta^{\prime}\right) \cdot C_{t}<0$ as required. In either case, the contraction exists by Lemma 3.16 and the existence of $\left(K_{X}+D\right)$-negative divisorial contractions.

If $\operatorname{loc}(R)=C$ is a curve then the result follows by Lemma 6.3.

\section{Special termination}

The goal of this section is to show the following:

Theorem 7.1 (Special termination) Let $X$ be a $\mathbb{Q}$-factorial quasi-projective threefold and let $\mathcal{F}$ be a co-rank one foliation with non-dicritical singularities on $X$. Let $(\mathcal{F}, \Delta)$ be an $F$-dlt pair. Let

$$
X=X_{0} \rightarrow X_{1} \rightarrow X_{2} \rightarrow \cdots
$$

be an infinite sequence of $\left(K_{\mathcal{F}}+\Delta\right)$-flips and let $\left(\mathcal{F}_{i}, \Delta_{i}\right)$ be the transformed foliated pair on $X_{i}$.

Then after finitely many flips, the flipping and flipped locus are disjoint from any lc centres of $\left(\mathcal{F}_{i}, \Delta_{i}\right)$.

Note that the result and the some of the proofs below were inspired by Shokurov's special termination in the classical setting [40] (see also [13, Sect. 4.2]).

We begin with the following:

Lemma 7.2 Let $X$ be a $\mathbb{Q}$-factorial quasi-projective threefold and let $\mathcal{F}$ be a co-rank one foliation with non-dicritical singularities on $X$. Let $(\mathcal{F}, \Delta)$ be an $F$-dlt pair. Suppose that there exist infinitely many $\mathcal{F}$-invariant divisors.

Then any sequence of $\left(K_{\mathcal{F}}+\Delta\right)$-flips terminates.

Proof Since the intersection of two invariant divisors is contained in Sing $\mathcal{F}$ and since $\mathcal{F}$ has non-dicritical singularities, it follows that there exist infinitely many pairwise disjoint $\mathcal{F}$-invariant divisors. By [35, Theorem 2], there exists a morphism $f: X \rightarrow C$ onto a smooth curve $C$ such that $\mathcal{F}$ is induced by $f$ and, in particular,

$$
K_{\mathcal{F}}=K_{X / C}+\sum\left(1-\ell_{D}\right) D
$$


where the sum is taken over all the vertical irreducible divisors and $\ell_{D}$ denotes the multiplicity of the fibre $f^{-1}(f(D))$ along $D$. Thus

$$
K_{\mathcal{F}} \sim_{\mathbb{Q}, f} K_{X}+\Gamma
$$

where $\Gamma$ is the sum of all the vertical prime divisors which are contained in a non-reduced fibre. Since $(\mathcal{F}, \Delta)$ is F-dlt and since any component of $\Gamma$ is $\mathcal{F}$-invariant, Lemma 3.16 implies that $(X, \Delta+\Gamma)$ is $\log$ canonical.

Note that if $X \rightarrow X^{\prime}$ is a $\left(K_{\mathcal{F}}+\Delta\right)$-flip and the flipping curve $\xi$ is horizontal, then $F \cdot \xi>0$ for any general fibre $F$ of $f$ and, in particular, the strict transform $F^{\prime}$ of $F$ on $X^{\prime}$ contains the flipped curve $\xi^{\prime}$, contradicting the fact that the transformed foliation $\mathcal{F}^{\prime}$ on $X^{\prime}$ has non-dicritical singularities (cf. Lemma 3.33). Thus, we may assume that any sequence of $\left(K_{\mathcal{F}}+\Delta\right)$ flips preserves the fibration onto the curve $C$. In particular, any sequence of $\left(K_{\mathcal{F}}+\Delta\right)$-flips is also a sequence of $\left(K_{X}+\Delta+\Gamma\right)$-flips. Thus, termination follows from termination of three-dimensional log canonical flips (cf. [40, Theorem 2.3]).

Thus, from now on, we assume that $\mathcal{F}$ admits at most finitely many invariant divisors. The proof proceeds in two steps. We first consider the case of lc centres transverse to the foliation. We then handle the case of lc centres tangent to the foliation by induction on dimension: supposing the statement is true for all $(d-1)$-dimensional lc centres, we then prove it for all $d$-dimensional lc centres.

\subsection{Log canonical centres transverse to the foliation}

Proposition 7.3 Let $X$ be a $\mathbb{Q}$-factorial quasi-projective threefold and let $\mathcal{F}$ be a co-rank one foliation with non-dicritical singularities on $X$. Suppose that $(\mathcal{F}, \Delta)$ is $F$-dlt and let $W \subset X$ be an lc centre transverse to $\mathcal{F}$. Let $\phi: X \rightarrow X^{+}$be a flip and let $\Delta^{+}=\phi_{*} \Delta$. Suppose that $W$ is not contained in the flipping locus $Z$. Let $W^{+}$be the strict transform of $W$ and $Z^{+}$be the flipped locus.

Then $W^{+} \cap Z^{+} \subset W^{+}$is not a divisor.

Proof Suppose for sake of contradiction that $W^{+} \cap Z^{+}=: D \subset W^{+}$is a divisor.

By Remark 3.10 it follows that $W$ is contained in the support of $\lfloor\Delta\rfloor$, and in fact is a stratum of $\lfloor\Delta\rfloor$. Hence $W^{+}$is a stratum of $\left\lfloor\Delta^{+}\right\rfloor$.

Let $\mathcal{F}^{+}$be the transformed foliation on $X^{+}$and let $\mathcal{G}^{+}$be the foliation restricted to $W^{+, v}$ where $v: W^{+, v} \rightarrow W^{+}$is the normalisation. Thus, since $W^{+}$is a stratum of $\left\lfloor\Delta^{+}\right\rfloor$, we may apply Lemma 3.18 and by induction on the 
codimension of $W^{+}$, we may write

$$
\left.\left(K_{\mathcal{F}^{+}}+\Delta^{+}\right)\right|_{W^{+, v}}=K_{\mathcal{G}^{+}}+\Theta^{+}
$$

where $\left(\mathcal{G}^{+}, \Theta^{+}\right)$is $\log$ canonical. Likewise we may write $\left.\left(K_{\mathcal{F}}+\Delta\right)\right|_{W^{v}}=$ $K_{\mathcal{G}}+\Theta$.

We now claim that $D$ is $\mathcal{G}^{+}$invariant.

If $\operatorname{dim}(W)=2$ then $D$ is a curve and it must be tangent to $\mathcal{F}^{+}$by Lemma 3.33, and hence it is invariant by $\mathcal{G}^{+}$. Indeed, by Lemma 2.7 we know that $\mathcal{F}^{+}$is terminal at the generic point of $D$ and so by Lemma 3.14 there exists a unique germ $S$ of an invariant surface containing $D$. It follows that $v^{-1}\left(S \cap W^{+}\right)$is $\mathcal{G}^{+}$-invariant and so $D$ is also $\mathcal{G}^{+}$-invariant.

If $\operatorname{dim}(W)=1$ then $D$ is a finite collection of points and $\mathcal{G}^{+}$is the foliation by points on $W^{+}$and so $D$ is automatically $\mathcal{G}^{+}$-invariant.

By Lemma 2.7, applied to the map $W^{v} \rightarrow W^{+, v}$, we know that $a(D, \mathcal{G}, \Theta)<a\left(D, \mathcal{G}^{+}, \Theta^{+}\right)$. However, since $\left(\mathcal{G}^{+}, \Theta^{+}\right)$is log canonical and since $D$ is invariant it follows that $a\left(D, \mathcal{G}^{+}, \Theta^{+}\right)=0$, i.e., the coefficient of $D$ in $\Theta^{+}$is zero, see Remark 2.3. On the other hand, since $(\mathcal{G}, \Theta)$ is log canonical, we have $a(D, \mathcal{G}, \Theta) \geq-\epsilon(D)=0$. This, however, is an impossibility.

Corollary 7.4 Let $X$ be a $\mathbb{Q}$-factorial quasi-projective threefold and let $\mathcal{F}$ be a co-rank one foliation with non-dicritical singularities on $X$. Suppose that $(\mathcal{F}, \Delta)$ is $F$-dlt.

Then, after finitely many flips, the flipping locus is disjoint from all the lc centres transverse to the foliation.

Proof Remark 3.10 implies that there are only finitely many lc centres transverse to $\mathcal{F}$ and these are strata of $\lfloor\Delta\rfloor$. Thus, by Lemma 2.7, we may assume that no lc centre transverse to the foliation is contained in the flipping locus. Let $\phi: X \rightarrow X^{+}$be a flip. Suppose that the flipping locus meets some lc centre of $(\mathcal{F}, \Delta)$ transverse to the foliation. Then, since these are strata of $\lfloor\Delta\rfloor$, it follows that the flipping locus meets some divisorial component $W$ of $\lfloor\Delta\rfloor$. Thus, to prove our result it suffices to show that, for any component $W$ of $\lfloor\Delta\rfloor$, after finitely many flips the flipping locus is disjoint from $W$.

So suppose that $W$ meets the flipping locus. Note that by Lemma 3.16 we see that $(X, \Delta)$ is dlt and so by [25, Corollary 5.52] it follows that $W$ is normal. Let $\psi: W \rightarrow W^{+}$be the induced map. Let $\mathcal{G}$ denote the restricted foliation and write

$$
\left.\left(K_{\mathcal{F}}+\Delta\right)\right|_{W}=K_{\mathcal{G}}+\Theta
$$

where by Lemma $3.18,(\mathcal{G}, \Theta)$ is F-dlt. In particular, $W$ is klt. 
By Proposition 7.3, none of the curves in the flipped locus is contained in $W^{+}$. Thus, $\psi$ is a birational contraction. If $\psi$ does not contract any divisors, then there exists a curve $Z$ contained in the flipping locus, such that $Z \cap W \neq \emptyset$ but $Z$ is not contained in $W$. Then $Z \cdot W>0$ and so if $Z^{+}$is a flipped curve we must have $Z^{+} \cdot W^{+}<0$ implying that $Z^{+} \subset W^{+}$, a contradiction.

Thus, $\psi$ contracts a divisor at each flip. In particular, each flip reduces $\rho(W)$ by 1 and we can only have finitely many such flips.

By Corollary 7.4, it suffices to show that the flipping locus is eventually disjoint from lc centres which are tangent to the foliation.

Lemma 7.5 After a finite sequence of flips, if $Z$ is an lc centre and $C$ is a flipping curve then $Z$ is not contained in $C$.

Proof By Corollary 7.4, after finitely many flips we may assume that the flipping locus is disjoint from all lc centres transverse to the foliation, in particular, it is disjoint from $\lfloor\Delta\rfloor$.

By Proposition 3.9, there are only finitely many lc centres of $(\mathcal{F}, \Delta)$ not contained in $\lfloor\Delta\rfloor$ and so the claim follows from Lemma 2.7.

Definition 7.6 (Hyperstandard set) Let $I \subset[0,1]$ be a subset. We define:

$$
I_{+}=\left\{\sum_{j=1}^{m} a_{j} i_{j} \mid i_{j} \in I, a_{j} \in \mathbb{N} \text { for } j=1, \ldots, m\right\} \cap[0,1],
$$

and

$$
D(I)=\left\{\frac{m-1+f}{m} \mid m \in \mathbb{N}, f \in I_{+}\right\} \cap[0,1] .
$$

Note that $D(D(I))=D(I)$ [33, Lemma 4.4]. Moreover, if $I \subset[0,1]$ is a finite set, then the only accumulation point of $D(I)$ is 1 and, in particular, it satisfies DCC.

Lemma 7.7 Let $(X, \Delta)$ be a log canonical pair such that the coefficients of $\Delta$ belong to a subset $I \subset[0,1]$, and let $S$ be an irreducible component of $\lfloor\Delta\rfloor$. Let $v: S^{\nu} \rightarrow S$ be the normalisation and let $\Theta$ be the divisor on $S^{\nu}$ defined by adjunction:

$$
\left.\left(K_{X}+\Delta\right)\right|_{S^{\nu}}=K_{S^{\nu}}+\Theta .
$$

Then, the coefficients of $\Theta$ belong to $D(I)$.

Proof Let $\pi: X^{\prime} \rightarrow X$ be a dlt blow up (X, $\Delta$ ), see [20, Theorem 10.4], and write $K_{X^{\prime}}+\Delta^{\prime}=\pi^{*}\left(K_{X}+\Delta\right)$. Note that, after possibly replacing $I$ by 
$I \cup\{1\}$, the coefficients of $\Delta^{\prime}$ belong to $I$ and that every irreducible component of $\lfloor\Delta\rfloor$ is normal. We may freely replace $(X, \Delta)$ by $\left(X^{\prime}, \Delta^{\prime}\right)$ and so we may assume without loss of generality that $S$ is normal. The result is then a direct consequence of [33, Lemma 4.3].

Given a F-dlt pair $(\mathcal{F}, \Delta)$ on a $\mathbb{Q}$-factorial quasi-projective threefold $X$, we denote by $d$ the minimal dimension of an lc centre of $(\mathcal{F}, \Delta)$ which is tangent to $\mathcal{F}$ and intersects the flipping locus of a $\left(K_{\mathcal{F}}+\Delta\right)$-flip. Our goal is to show that there can be only finitely many flips with $d=0,1$ or 2 .

\subsection{Special termination in dimension $d=0$}

This follows directly from Lemma 7.5

\subsection{Special termination in dimension $d=1$}

Lemma 7.8 Set up as above. Then after finitely many flips the flipping locus is disjoint from all one-dimensional lc centres.

Proof Let $I$ be the set of coefficients of $\Delta$. Recall that $D(I)$ satisfies DCC. Let $C$ be a one-dimensional lc centre which is tangent to $\mathcal{F}$ and let $v: C^{\nu} \rightarrow C$ be its normalisation. Let $\Theta \geq 0$ be the $\mathbb{Q}$-divisor on $C^{v}$ whose existence is guaranteed by Lemma 3.22. Since $D(D(I))=D(I)$, it follows by Lemma 7.7 and the proof of Lemma 3.22 that the coefficients of $\{\Theta\}$ take values in $D(I)$.

It follows by Lemma 2.7 that, after a flip, $\{\Theta\}$ strictly decreases. However, by Lemma 3.22 and since we are assuming that there are no zero-dimensional lc centre intersecting the flipping locus, it follows that the flip is an isomorphism near $\lfloor\Theta\rfloor$ and the result follows.

\subsection{Special termination in dimension $d=2$}

Lemma 7.9 Set up as above. Then after finitely many flips the flipping locus is disjoint from all two-dimensional lc centres.

Proof Let $I \subset[0,1]$ be a finite set containing the coefficients of $\Delta$. Let $S$ be a two-dimensional lc centre intersecting the flipping locus. By Corollary 7.4, we may assume that $S$ is $\mathcal{F}$-invariant and, by Lemma 3.18, we may write $\left.\left(K_{\mathcal{F}}+\Delta\right)\right|_{W}=K_{W}+\Theta$ for some $\mathbb{Q}$-divisor $\Theta \geq 0$ where $W \rightarrow S$ is the normalisation and such that $\left(W, \Theta^{\prime}:=\lfloor\Theta\rfloor_{\text {red }}+\{\Theta\}\right)$ is lc and $\left(W,(1-\epsilon) \Theta^{\prime}\right)$ is klt for $0<\epsilon<1$. Note that the coefficients of $\Theta^{\prime}$ belong to $D(I)$. 
We define

$$
d_{I}(W, \Theta)=\sum_{a \in D(I)} \#\left\{E \mid a(E, W, \Theta)<-a \text { and } c_{X}(E) \not \subset\left\lfloor\Theta^{\prime}\right\rfloor\right\} .
$$

Then $d_{I}(W, \Theta)<\infty$.

Let $\phi: X \rightarrow X^{+}$be a flip, and let $\psi: S \rightarrow S^{+}$be the induced birational map. We denote by $\left(\mathcal{F}^{+}, \Delta^{+}\right)$be the transformed foliated pair and we write $\left.\left(K_{\mathcal{F}^{+}}+\Delta^{+}\right)\right|_{W^{+}}=K_{W^{+}}+\Theta^{+}$where $W^{+} \rightarrow S^{+}$is the normalisation. Note that $d_{I}(W, \Theta) \geq d_{I}\left(W^{+}, \Theta^{+}\right)$.

Suppose first that $\psi^{-1}$ contracts a divisor $D \subset S^{+}$. Let $Z \subset S$ be the centre of $D$ on $S$. By induction we know that $Z$ is not contained in $\left\lfloor\Theta^{\prime}\right\rfloor$. It follows that $d_{I}\left(W^{+}, \Theta^{+}\right)<d_{I}(W, \Theta)$.

Thus, after finitely many flips, we may assume that $\psi$ is a birational contraction. As in the proof of Corollary 7.4, the claim follows.

The Lemma above concludes the proof of Theorem 7.1.

Corollary 7.10 Let $X$ be a $\mathbb{Q}$-factorial quasi-projective threefold and let $\mathcal{F}$ be a co-rank 1 foliation with non-dicritical singularities on $X$. Let $\pi: X \rightarrow Z$ be a birational morphism. Let $(\mathcal{F}, \Delta)$ be an $F$-dlt pair on $X$. Assume that every component of $\operatorname{exc}(\pi)$ is an lc centre for $(\mathcal{F}, \Delta)$.

Then any sequence of $\left(K_{\mathcal{F}}+\Delta\right)$-flips over $Z$

$$
X=X_{0} \rightarrow X_{1} \rightarrow X_{2} \rightarrow \cdots
$$

terminates.

Proof By Theorem 7.1, any sequence of flips is eventually disjoint from the lc centres of $(\mathcal{F}, \Delta)$ and so is eventually disjoint from $\operatorname{exc}(\pi)$, in which case the MMP terminates.

\section{Existence of F-dlt modifications}

We now show the existence of an F-dlt modification as in Definition 3.28. The result is a consequence of the existence of flips and special termination and it will be used to prove the base point free theorem in Sect. 9 .

Theorem 8.1 (Existence of F-dlt modifications) Let $\mathcal{F}$ be a co-rank one foliation on a normal projective variety $X$ of dimension at most three. Let $(\mathcal{F}, \Delta)$ be a foliated pair.

Then $(\mathcal{F}, \Delta)$ admits a F-dlt modification $\pi: Y \rightarrow X$ (cf. Definition 3.28) such that if $\mathcal{G}$ is the pulled back foliation on $Y$ then

1. $Y$ is $\mathbb{Q}$-factorial, 
2. $Y$ is klt and

3. $\mathcal{G}$ is non-dicritical.

Suppose in addition that $(\mathcal{F}, \Delta)$ is lc and let $\Gamma:=\pi_{*}^{-1} \Delta+\sum \epsilon\left(E_{i}\right) E_{i}$ where the sum is over all the $\pi$-exceptional divisors. Then we may choose $\pi: Y \rightarrow X$ so that if $Z$ is an lc centre of $(\mathcal{G}, \Gamma)$ then $Z$ is contained in a codimension one lc centre of $(\mathcal{G}, \Gamma)$.

Proof We assume that $\operatorname{dim} X=3$. The case where $\operatorname{dim} X=2$ is similar. Let $\phi: W \rightarrow X$ be a foliated $\log$ resolution. Let $\mathcal{H}$ be the pulled back foliation on $W$.

We may write

$$
K_{\mathcal{H}}+\sum b_{i} G_{i}+\sum a_{j} F_{j}+\phi_{*}^{-1} \Delta=\phi^{*}\left(K_{\mathcal{F}}+\Delta\right),
$$

where $G_{i}, F_{j}$ are $\phi$-exceptional prime divisors such that $b_{i} \geq \epsilon\left(G_{i}\right)$ and $a_{j}<\epsilon\left(F_{j}\right)$. Let

$$
\widetilde{\Delta}=\sum b_{i} G_{i}+\sum a_{j} F_{j}+\phi_{*}^{-1} \Delta
$$

and

$$
\Theta=\sum \epsilon\left(G_{i}\right) G_{i}+\sum \epsilon\left(F_{j}\right) F_{j}+\phi_{*}^{-1} \Delta .
$$

Note that $(\mathcal{H}, \Theta)$ is F-dlt. By Proposition 3.9, after possibly replacing $W$ by a sufficiently high log resolution, we may assume that every lc centre is contained in a codimension one lc centre.

We run a $\left(K_{\mathcal{H}}+\Theta\right)$-MMP over $X$. Recall that, by Remark 2.13, simple singularities are non-dicritical. By Theorem 6.7 all the required divisorial contractions exists and by Theorem 6.4 all the flips exist. By construction each $G_{i}, F_{j}$ is an lc centre of $(\mathcal{H}, \Theta)$ and so Corollary 7.10 implies that this MMP terminates. Call this MMP $f: W \rightarrow Y$ and let $\mathcal{G}$ be the transformed foliation on $Y$. Note that Lemma 3.11 implies that $\left(\mathcal{G}, f_{*} \Theta\right)$ is F-dlt.

The MMP preserves $\mathbb{Q}$-factoriality, klt singularities (cf. Theorems 6.4, 6.7) and non-dicriticality (cf. Lemma 3.33), and so we have that $Y$ is $\mathbb{Q}$-factorial and klt and $\mathcal{G}$ is non-dicritical.

Denote by $\pi: Y \rightarrow X$ the induced morphism. We have $K_{\mathcal{G}}+f_{*} \widetilde{\Delta}=$ $\pi^{*}\left(K_{\mathcal{F}}+\Delta\right)$ and so $D:=f_{*} \Theta-f_{*} \widetilde{\Delta}$ is $\pi$-nef and $\pi$-exceptional. The negativity lemma then implies that $f_{*} \widetilde{\Delta}-f_{*} \Theta=-D \geq 0$. Thus, setting $F=-D$ and noting that $f_{*} \Theta=\pi_{*}^{-1} \Delta+\sum \epsilon\left(E_{i}\right) E_{i}$ where we sum over the $\pi$-exceptional divisors, we have

$$
K_{\mathcal{G}}+\pi_{*}^{-1} \Delta+\sum \epsilon\left(E_{i}\right) E_{i}+F=\pi^{*}\left(K_{\mathcal{F}}+\Delta\right) .
$$


We now show the last claim. To this end, we may freely replace $(\mathcal{F}, \Delta)$ by $\left(\mathcal{G}, f_{*} \Theta\right)$ and so we may assume that $(\mathcal{F}, \Delta)$ is F-dlt. Arguing as above we see that it suffices to show that the $\left(K_{\mathcal{H}}+\Theta\right)$-MMP does not contract any component in the support of $\sum G_{i}$. By assumption, $b_{i}=\epsilon\left(G_{i}\right)$ and so

$$
\left(K_{\mathcal{H}}+\Theta\right)-\left(K_{\mathcal{H}}+\widetilde{\Delta}\right)=\sum\left(\epsilon\left(F_{j}\right)-a_{j}\right) F_{j} \geq 0 .
$$

Since $K_{\mathcal{H}}+\widetilde{\Delta}$ is trivial over $X$, each step of the $\left(K_{\mathcal{H}}+\Theta\right)$-MMP is $\sum\left(\epsilon\left(F_{j}\right)-\right.$ $\left.a_{j}\right) F_{j}$-negative and so only components in the support of $\sum\left(\epsilon\left(F_{j}\right)-a_{j}\right) F_{j}$ are contracted. In particular, no component in the support of $\sum E_{i}$ is contracted by the MMP and our result follows.

Theorem 8.2 (Cone theorem for lc pairs) Let X be a normal projective threefold and let $\mathcal{F}$ be a co-rank one foliation on $X$ with non-dicritical singularities. Suppose that $X$ is potentially klt. Let $(\mathcal{F}, \Delta)$ be an lc pair where $\Delta \geq 0$ and let $H$ be an ample $\mathbb{Q}$-divisor.

Then there exist countable many curves $\xi_{1}, \xi_{2}, \ldots$ such that

$$
\overline{N E(X)}=\overline{N E(X)}_{K_{\mathcal{F}}+\Delta \geq 0}+\sum \mathbb{R}_{+}\left[\xi_{i}\right] .
$$

Furthermore, for each $i$, $\xi_{i}$ is a rational curve tangent to $\mathcal{F}$ such that $\left(K_{\mathcal{F}}+\right.$ $\Delta) \cdot \xi \geq-6$, and if $C \subset X$ is a curve such that $[C] \in \mathbb{R}_{+}\left[\xi_{i}\right]$ and $\operatorname{loc}\left(\mathbb{R}_{+}\left[\xi_{i}\right]\right) \neq$ $X$ then $C$ is tangent to $\mathcal{F}$.

In particular, there exist only finitely many $\left(K_{\mathcal{F}}+\Delta+H\right)$-negative extremal rays.

Proof By Theorem 8.1, there exists a F-dlt modification $\pi: Y \rightarrow X$ for the foliated pair $(\mathcal{F}, \Delta)$. We may write $K_{\mathcal{G}}+\Gamma=\pi^{*}\left(K_{\mathcal{F}}+\Delta\right)$ where $\mathcal{G}$ is the transformed foliation on $Y$ and $\Gamma \geq 0$.

Observe that if $R \subset \overline{N E}(X)$ is an extremal ray then there exists an extremal ray $R^{\prime} \subset \overline{N E}(Y)$ such that $\pi_{*} R^{\prime}=R$. Moreover, if $R$ is $\left(K_{\mathcal{F}}+\Delta\right)$-negative then $R^{\prime}$ is $\left(K_{\mathcal{G}}+\Gamma\right)$-negative and so by Theorem $3.31 R^{\prime}$ is spanned by a rational curve $\xi$ tangent to $\mathcal{G}$ with $\left(K_{\mathcal{G}}+\Gamma\right) \cdot \xi \geq-6$. Then $\pi(\xi)$ spans $R$ and has all the desired properties.

If $C \subset X$ and $[C] \in \mathbb{R}_{+}\left[\xi_{i}\right]$ for some $i$ then we may apply Lemma 3.30 to conclude that $C$ is tangent to $\mathcal{F}$.

\subsection{Contraction in the non- $\mathbb{Q}$-factorial case}

Through out this subsection, we assume that $(X, \Gamma)$ is klt for some $\Gamma \geq 0$ but that $X$ is not necessarily $\mathbb{Q}$-factorial. 
Lemma 8.3 Let $X$ be a normal projective threefold and let $\mathcal{F}$ be a co-rank one foliation on $X$ with non-dicritical singularities. Suppose that $X$ is potentially klt. Let $\Delta \geq 0$ be a $\mathbb{Q}$-divisor such that $(\mathcal{F}, \Delta)$ is a log canonical pair, and suppose there exists a small $\mathbb{Q}$-factorialisation $\pi: \bar{X} \rightarrow X($ cf. Definition 2.22) such that if we write $K_{\overline{\mathcal{F}}}+\bar{\Delta}=\pi^{*}\left(K_{\mathcal{F}}+\Delta\right)$, where $\overline{\mathcal{F}}$ is the pulled back foliation on $\bar{X}$, then for any choice of $\epsilon>0$ we may find $\Theta$ such that $(1-\epsilon) \bar{\Delta} \leq$ $\Theta \leq \bar{\Delta}$ and $(\overline{\mathcal{F}}, \Theta)$ is F-dlt.

Let $R$ be a $\left(K_{\mathcal{F}}+\Delta\right)$-negative extremal ray. Assume that $\operatorname{loc}(R) \neq X$.

Then there exists a contraction

$$
\phi_{R}: X \rightarrow \mathcal{Y}
$$

of $R$ in the category of algebraic spaces.

Proof By Theorem 8.2, there exists a nef $\mathbb{Q}$-Cartier divisor $H_{R}$ on $X$ which defines a supporting hyperplane for $R$ in $\overline{N E}(X)$. Let $\pi: \bar{X} \rightarrow X$ be a small $\mathbb{Q}$-factorialisation of $X$ as in the hypotheses of the Lemma.

First, suppose that $\operatorname{loc}(R)=D$ is a divisor. By Theorem 8.2, we may find $\epsilon>0$ sufficiently small and a $\mathbb{Q}$-divisor $\Theta$ as in the hypotheses of the Lemma so that $K_{\overline{\mathcal{F}}}+\Theta$ is negative on any extremal ray $R^{\prime}$ in $\overline{N E}(\bar{X})$ such that $\pi_{*} R^{\prime}=R$.

Suppose that $D$ contains an irreducible component $D_{0}$ transverse to the foliation. Let $\overline{D_{0}}$ be the strict transform of $D_{0}$ under $\pi$ and let $v:{\overline{D_{0}}}^{v} \rightarrow \overline{D_{0}}$ the normalisation map. We may write

$$
v^{*}\left(K_{\overline{\mathcal{F}}}+\bar{\Delta}+\overline{D_{0}}\right)=K_{\mathcal{G}}+\Gamma
$$

where $\mathcal{G}$ is the restricted foliation and $\Gamma \geq 0$. It follows that ${\overline{D_{0}}}^{v}$ is covered by $\left(K_{\mathcal{G}}+\Gamma\right)$-negative curves and, therefore, $K_{\mathcal{G}}+\Gamma$ is not pseudo-effective. Thus, [41, Corollary 2.28] implies that $\overline{D_{0}}$ ' is covered by rational curves which are tangent to $\mathcal{G}$ and, in particular, $\mathcal{G}$ is algebraically integrable. Thus, since $\overline{\mathcal{F}}$ is non-dicritical, there exists a morphism ${\overline{D_{0}}}^{v} \rightarrow B$ onto a curve $B$ such that the general fibre is $\mathbb{P}^{1}$. In particular, if $F$ is a general fibre in this $\mathbb{P}^{1}$-fibration structure, then we claim that

$$
\left(K_{\overline{\mathcal{F}}}+\bar{\Delta}+\overline{D_{0}}\right) \cdot F=\left(K_{\bar{X}}+\bar{\Delta}+\overline{D_{0}}\right) \cdot F \text {. }
$$

Indeed, if we write $v^{*}\left(K_{X}+\bar{\Delta}+\overline{D_{0}}\right)=K_{D_{0}^{v}}+\Gamma^{\prime}$ then by Lemma 3.23 we see that the coefficient in $\Gamma$ and $\Gamma^{\prime}$ of a curve $\Sigma$ which is not a fibre of $D_{0}^{v} \rightarrow B$ is the same. In particular, it follows that $\Gamma \cdot F=\Gamma^{\prime} \cdot F$. Finally, observe that $K_{{\overline{D_{0}}}^{v}} \cdot F=K_{\mathcal{G}} \cdot F=-2$ and our stated equality follows. 
We run a $\left(K_{\overline{\mathcal{F}}}+\Theta\right)$-MMP contracting/flipping only $\pi^{*} H_{R}$-trivial extremal rays $R^{\prime}$ such that $\operatorname{loc}\left(R^{\prime}\right)$ meets the strict transform of $D$. Theorem 6.4 implies that all the required flips exist.

By our above observation we know that if the strict transform of $D$ contains a non-invariant component we may choose this extremal ray to also be $\left(K_{\bar{X}}+\Theta\right)$ negative. Thus, by classical termination of log flips we see that there can only be finitely many flips before the strict transform of each non-invariant component of $D$ is contracted. By Special Termination (cf. Theorem 7.1), there are only finitely many such flips before the strict transform of each invariant component of $D$ is contracted.

Denote by $\bar{Y}$ the step in this MMP after the last component of $D$ is contracted, and let $f: \bar{X} \rightarrow \bar{Y}$ denote the induced rational map. Observe that each step of this MMP is $\pi^{*} H_{R}$-trivial and so $\pi^{*} H_{R}$ descends to a $\mathbb{Q}$-Cartier divisor $M$ on $\bar{Y}$

We know that $\bar{Y}$ is $\mathbb{Q}$-factorial and, as in the proof of [41, Lemma 8.20], we know that if $S$ is a divisor on $\bar{Y}$ then $M^{2} \cdot S>0$. Moreover, if $B$ is a curve we see that $M \cdot B=0$ if and only if $B$ is the strict transform of a $\pi$-exceptional curve, $B$ is the strict transform of a flipped curve or $B$ is the strict transform of a curve $C$ with $[C] \in R$. Notice that there are finitely many such curves and let $\Sigma$ be the union of all such curves. By [41, Lemma 8.21] there exists a contraction of $\Sigma$ in the category of algebraic spaces, call it $c: \bar{Y} \rightarrow \mathcal{Y}$. Since $c$ contracts every $\pi$-exceptional curve and every flipped curve it gives a contraction

$$
\phi_{R}: X \rightarrow \mathcal{Y}
$$

Now suppose that $\operatorname{loc}(R)$ is a curve. In this case we see that if $S$ is a divisor on $\bar{X}$ then, as in the proof of [41, Lemma 8.20], we have that $\left(\pi^{*} H_{R}\right)^{2} \cdot S>0$ and that $\left(\pi^{*} H_{R}\right) \cdot C=0$ if and only if $C \subset \pi^{-1}(\operatorname{loc}(R)) \cup \operatorname{Exc} \pi$. As above, we apply [41, Lemma 8.21] to produce a contraction of $\pi^{-1}(\operatorname{loc}(R))$ in the category of algebraic spaces, which factors through $\pi$.

Theorem 8.4 Let $(X, \Gamma)$ be a projective three-dimensional klt pair and let $\mathcal{F}$ be a co-rank one foliation on $X$ with non-dicritical singularities. Let $\Delta \geq 0$ be a $\mathbb{Q}$-divisor such that $(\mathcal{F}, \Delta)$ is a log canonical pair and suppose there exists a small $\mathbb{Q}$-factorialisation $\pi: \bar{X} \rightarrow X$ such that if we write $K_{\overline{\mathcal{F}}}+\bar{\Delta}=$ $\pi^{*}\left(K_{\mathcal{F}}+\Delta\right)$, where $\overline{\mathcal{F}}$ is the pulled back foliation on $\bar{X}$, then for any choice of $\epsilon>0$ we may find $\Theta$ such that $(1-\epsilon) \bar{\Delta} \leq \Theta \leq \bar{\Delta}$ and $(\overline{\mathcal{F}}, \Theta)$ is F-dlt. Let $R$ be a $\left(K_{\mathcal{F}}+\Delta\right)$-negative extremal ray. Assume that $\operatorname{loc}(R) \neq X$.

Then the contraction associated to $R$

$$
\phi_{R}: X \rightarrow Y
$$


exists in the category of projective varieties and $\rho(X / Y)=1$. In particular, $\phi_{R}$ is extremal.

Note that, by Lemma 3.29, the assumption of the Theorem are verified if $(\mathcal{F}, \Delta)$ is F-dlt.

Proof Let $\phi_{R}: X \rightarrow \mathcal{Y}$ be the contraction onto an algebraic space $\mathcal{Y}$, whose existence is guaranteed by Lemma 8.3. We first show that if $M$ is a $\mathbb{Q}$-Cartier divisor with $M \cdot R=0$ then $M=\phi_{R}^{*} N$ for some $\mathbb{Q}$-Cartier divisor on $\mathcal{Y}$. Observe that this problem is étale local on $\mathcal{Y}$, so we may freely replace $\mathcal{Y}$ by a sufficiently small étale neighborhood of some point $y \in \mathcal{Y}$.

Let $\pi: \bar{X} \rightarrow X$ be a small $\mathbb{Q}$-factorialisation of $X$ as in the hypotheses of the Lemma and let $g: \bar{X} \rightarrow \mathcal{Y}$ be the composition of $\pi$ with $\phi_{R}$. We may write

$$
K_{\overline{\mathcal{F}}}+\bar{\Delta}=\pi^{*}\left(K_{\mathcal{F}}+\Delta\right) \text { and } K_{\bar{X}}+\bar{\Gamma}=\pi^{*}\left(K_{X}+\Gamma\right) .
$$

By Lemma 5.2, we may replace $\mathcal{Y}$ by another étale neighborhood of $y \in \mathcal{Y}$, so that we may approximate every invariant divisor (formal or otherwise) of $\overline{\mathcal{F}}$ meeting $g^{-1}(y)$ by global divisors on $\bar{X}$. Let $S_{k}$ be the collection of all such divisors. As in Sect. 6.1 we see that

$$
\left(K_{\overline{\mathcal{F}}}+\bar{\Delta}\right)-\left(K_{\bar{X}}+\bar{\Delta}+\sum S_{k}\right)
$$

is $g$-nef.

For some $\epsilon>0$ sufficiently small, we may run a $\left(K_{\bar{X}}+\bar{\Gamma}+\epsilon\left(\bar{\Delta}+\sum S_{k}\right)\right)$ MMP over $X$, and we obtain a map $\bar{X} \rightarrow \bar{X}^{\prime}$. Let $\pi^{\prime}: \bar{X}^{\prime} \rightarrow X$ be the induced morphism. Each step of this MMP is $\left(K_{\bar{X}}+\bar{\Gamma}\right)$-trivial and so if we let $T_{k}$ be the strict transform of $S_{k}$ and $\bar{\Delta}^{\prime}$ be the strict transform of $\bar{\Delta}$ on $\bar{X}^{\prime}$, we see that $\bar{\Delta}^{\prime}+\sum T_{k}$ is nef over $X$. Observe that $\sum T_{k}$ still approximates the divisors which are invariant with respect to the transformed foliation $\overline{\mathcal{F}}^{\prime}$ on $\bar{X}^{\prime}$.

Thus, replacing $\bar{X}$ by $\bar{X}^{\prime}$ we may freely assume that $\left(\Delta+\sum S_{k}\right) \cdot C \geq 0$ for any $\pi$-exceptional curve $C$. Since $K_{\mathcal{F}}+\Delta$ is strictly negative on any $\phi_{R}$-exceptional curve, we see that for $0<\delta \ll 1$ we have

$$
-\left(K_{\bar{X}}+(1-\delta)\left(\bar{\Delta}+\sum S_{k}\right)\right)
$$

is nef over $\mathcal{Y}$. By Lemma 3.16 and the fact that $(\overline{\mathcal{F}}, \bar{\Delta})$ is log canonical we see that $\left(\bar{X}, \bar{\Delta}+\sum S_{k}\right)$ is $\log$ canonical and since $\bar{X}$ is klt we have that $\left(\bar{X},(1-\delta)\left(\bar{\Delta}+\sum S_{k}\right)\right)$ is klt for $\delta>0$ and so we may apply the base point free theorem to $\pi^{*} M$ to conclude that there exists a $\mathbb{Q}$-Cartier divisor $N$ on $\mathcal{Y}$ with $\phi_{R}^{*} N=M$. 
Let $H_{R}$ be a nef $\mathbb{Q}$-Cartier divisor on $X$ which defines a supporting hyperplane for $R$ in $\overline{N E}(X)$. Taking $M=H_{R}$ we see that by applying the Nakai-Moishezon criterion for algebraic spaces (cf. [26, Theorem 3.11]) to $N$ that $N$ is ample and hence $\mathcal{Y}=Y$ is projective.

Observe that, by Lemma 3.29, the hypotheses of Theorem 8.4 are satisfied if we suppose that $(\mathcal{F}, \Delta)$ is F-dlt.

\subsection{Potentially klt varieties}

Lemma 8.5 Let $X$ be a normal projective variety. Suppose that $X$ is étale locally potentially klt. Then $X$ is potentially klt.

In particular, let $\phi_{R}: X \rightarrow Y$ be the contraction associated to an extremal ray as in Theorem 8.4. Then $Y$ is potentially klt.

Proof Choose a finite étale cover

$$
\left\{g_{i}: U_{i} \rightarrow X\right\}_{i=1, \ldots, N}
$$

such that $U_{i}$ is affine and there exists $\Delta_{i} \geq 0$ such that $\left(U_{i}, \Delta_{i}\right)$ is klt.

Without loss of generality we may assume each $g_{i}$ is Galois, with Galois group $G_{i}$. Perhaps replacing $\Delta_{i}$ by $\frac{1}{\# G_{i}} \sum_{g \in G_{i}} g \cdot \Delta_{i}$ we may assume that there exists a Zariski open set $V_{i} \subset X$ and a $\mathbb{Q}$-divisor $\Theta_{i} \geq 0$ on $V_{i}$ such that $g_{i}$ factors through $V_{i}, K_{V_{i}}+\Theta_{i}$ is $\mathbb{Q}$-Cartier and $g_{i}^{*}\left(K_{V_{i}}+\Theta_{i}\right)=K_{U_{i}}+\Delta_{i}$. Observe that $\left(V_{i}, \Theta_{i}\right)$ is klt and so we may freely assume that $g_{i}: U_{i} \rightarrow X$ is an open immersion.

There exists $m>0$ such that $m \Delta_{i} \in\left|-m K_{U_{i}}\right|$ for all $i$. Let $H$ be a divisor on $X$ such that $\mathcal{O}\left(-m K_{X}+m H\right)$ is globally generated. We may assume that, for all $i$, there exists $D_{i} \in\left|-m K_{X}+m H\right|$ such that $\left(U_{i},\left.\frac{1}{m} D_{i}\right|_{U_{i}}\right)$ is klt. It follows that, for a general element $D \in\left|-m K_{X}+m H\right|$, we have that $\left(X, \frac{1}{m} D\right)$ is klt. Thus, $X$ is potentially klt.

To prove our final claim it suffices to check that $Y$ is étale locally potentially klt. So let $y \in Y$ and let $U$ be a sufficiently small étale neighborhood of $y$, and let $X_{U}=X \times{ }_{Y} U$. By the construction given in proof of Theorem 8.4, there exists a small morphism $\pi: \overline{X_{U}} \rightarrow X_{U}$ and a divisor $D \geq 0$ such that $\left(\overline{X_{U}}, D\right)$ is klt and $-\left(K_{\overline{X_{U}}}+D\right)$ is $\phi_{R}$-nef. By the basepoint free theorem, we may find a $0 \leq A \sim_{\mathbb{Q}}-\left(K_{\overline{X_{U}}}+D\right)$ such that $\left(\overline{X_{U}}, D+A\right)$ is klt and $K_{\overline{X_{U}}}+D+A$ is $\phi_{R}$-trivial. Thus, $\left(U,\left(\phi_{R}\right)_{*}(D+A)\right)$ is klt and so $X$ is étale locally potentially klt. 


\section{Base point free theorem}

The goal of this section is to prove the base point free theorem. We begin with the following version of the canonical bundle formula:

Lemma 9.1 Let $X$ be a normal projective variety of dimension at most three and let $(\mathcal{F}, \Delta)$ be an lc pair on $X$ such that $\Delta \geq 0$. Suppose that there is a fibration $f: X \rightarrow Y$ onto a variety $Y$ with klt singularities and such that the general fibre of $f$ is tangent to $\mathcal{F}$. Assume that $K_{\mathcal{F}}+\Delta \sim_{\mathbb{Q}, f} 0$.

Then there is a foliation $\mathcal{G}$ on $Y$ such that $f^{-1} \mathcal{G}=\mathcal{F}$, and a $\mathbb{Q}$-divisor $\Theta \geq 0$ and a semi-ample divisor $D$ such that $K_{\mathcal{F}}+\Delta \sim_{\mathbb{Q}} f^{*}\left(K_{\mathcal{G}}+\Theta+D\right)$ and $(\mathcal{G}, \Theta)$ is $l c$.

Proof First, notice that since the fibres of $f$ are tangent to $\mathcal{F}$ there exists a foliation $\mathcal{G}$ on $Y$ so that $\mathcal{F}=f^{-1} \mathcal{G}$. We also have that there exists $M$ on $Y$ so that $K_{\mathcal{F}}+\Delta \sim_{\mathbb{Q}} f^{*} M$.

Consider a commutative diagram as follows:

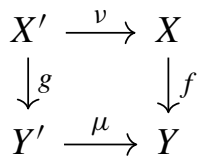

where $\mu, v$ are resolutions of singularities. Let $\mathcal{F}^{\prime}$ and $\mathcal{G}^{\prime}$ be the transformed foliations on $X^{\prime}$ and $Y^{\prime}$ respectively.

Write

$$
K_{\mathcal{F}^{\prime}}+\Delta^{\prime}=v^{*}\left(K_{\mathcal{F}}+\Delta\right) .
$$

and so we have $K_{\mathcal{F}^{\prime}}+\Delta^{\prime} \sim_{\mathbb{Q}} g^{*}\left(\mu^{*} M\right)$.

We claim that there exists an open subset $U \subset Y^{\prime}$ such that $Y^{\prime} \backslash U$ has codimension at least two and such that, over $U$ we have that $K_{\mathcal{F}^{\prime}} / \mathcal{G}^{\prime}=K_{X^{\prime} / Y^{\prime}}-$ $R$ where $R$ is $\mathcal{F}^{\prime}$-invariant and $K_{\mathcal{F}^{\prime} / G^{\prime}}:=K_{\mathcal{F}^{\prime}}-g^{*} K_{\mathcal{G}^{\prime}}$. Indeed, $R$ is supported in the zero locus of a 1-form obtained as the pull-back of a 1-form on $Y^{\prime}$ which defines $\mathcal{G}^{\prime}$. Thus, the claim follows. By [27, Theorem 8.3.7] we may find a nef $\mathbb{Q}$-divisor $J$ and an effective $\mathbb{Q}$-divisor $B$ such that $\mu^{*} M \sim_{\mathbb{Q}} K_{\mathcal{G}^{\prime}}+$ $B+J$. Furthermore, by [5, Theorem 0.1] in the case $\operatorname{dim}(Y)=1$ and [36, Theorem 8.1] when $\operatorname{dim}(Y)=2$ we know that $J$ is in fact semi-ample.

If $B=\sum a_{i} B_{i}$, then

$a_{i}=1-\sup \left\{t \mid\left(X^{\prime}, \Delta^{\prime}-R+\operatorname{tg}^{*} B_{i}\right)\right.$ is lc above the generic point of $\left.B_{i}\right\}$.

An explicit calculation shows that

$a_{i}=\epsilon\left(B_{i}\right)-\sup \left\{t \mid\left(\mathcal{F}^{\prime}, \Delta^{\prime}+\operatorname{tg}^{*} B_{i}\right)\right.$ is lc above the generic point of $\left.B_{i}\right\}$. 
Thus, since $\left(\mathcal{F}^{\prime}, \Delta^{\prime}\right)$ is lc, it follows that $a_{i} \leq \epsilon\left(B_{i}\right)$ and $\mu_{*} B \geq 0$.

Since $Y$ is klt of dimension at most two, it follows that $Y$ is $\mathbb{Q}$-factorial. Since $J$ is semi-ample the base locus of $\mu_{*} J$ consists of isolated points. Thus, Fujita Theorem [19, Theorem 1.10] implies that $\mu_{*} J$ is semi-ample. Letting $\Theta=\mu_{*} B$ and $D=\mu_{*} J$ gives our result.

Lemma 9.2 Let $X$ be a normal projective threefold and let $\mathcal{F}$ be a co-rank one foliation with non-dicritical singularities. Suppose that $(X, D)$ is klt for some $D \geq 0$. Let $\Delta=A+B$ be a $\mathbb{Q}$-divisor such that $(\mathcal{F}, \Delta)$ is an lc pair, $A \geq 0$ is an ample $\mathbb{Q}$-divisor and $B \geq 0$. Assume that $K_{\mathcal{F}}+\Delta$ is not nef, but there exists a $\mathbb{Q}$-divisor $H$ such that $K_{\mathcal{F}}+\Delta+H$ is nef. Let

$$
\lambda=\inf \left\{t>0 \mid K_{\mathcal{F}}+\Delta+t H \text { is nef }\right\} .
$$

Then there exists a $\left(K_{\mathcal{F}}+\Delta\right)$-negative extremal ray $R$ such that $\left(K_{\mathcal{F}}+\right.$ $\Delta+\lambda H) \cdot \xi=0$, for any $\xi \in R$.

Proof By Theorem 8.2, there exist only finitely many curves $\xi_{1}, \ldots, \xi_{m}$ such that if $R_{i}=\mathbb{R}_{+}\left[\xi_{i}\right]$ for $i=1, \ldots, m$, then $R_{1}, \ldots, R_{m}$ are $\left(K_{\mathcal{F}}+\Delta\right)$-negative extremal rays.

Let $C:=K_{\mathcal{F}}+\Delta+H$ and let

$$
\mu=\min _{i} \frac{C \cdot \xi_{i}}{-\left(K_{\mathcal{F}}+\Delta\right) \cdot \xi_{i}} .
$$

It follows easily that $\mu=\frac{1-\lambda}{\lambda}$. By construction, there exists $j$ such that

$$
\frac{1}{\lambda}\left(K_{\mathcal{F}}+\Delta+\lambda H\right) \cdot \xi_{j}=\left(\mu\left(K_{\mathcal{F}}+\Delta\right)+C\right) \cdot \xi_{j}=0 .
$$

Thus, the claim follows by taking $R=R_{j}$.

Lemma 9.3 Let $X$ be a normal projective threefold and let $\mathcal{F}$ be a co-rank one foliation with non-dicritical singularities. Suppose that $X$ is potentially klt. Let $\Delta \geq 0$ be a $\mathbb{Q}$-divisor such that $(\mathcal{F}, \Delta)$ is a log canonical pair, and suppose there exists a small $\mathbb{Q}$-factorialisation $\pi: \bar{X} \rightarrow X$ such that if we write $K_{\overline{\mathcal{F}}}+\bar{\Delta}=\pi^{*}\left(K_{\mathcal{F}}+\Delta\right)$, where $\overline{\mathcal{F}}$ is the pulled back foliation on $\bar{X}$, then for any choice of $\epsilon>0$ we may find $\Theta$ such that $(1-\epsilon) \bar{\Delta} \leq \Theta \leq \bar{\Delta}$ and $(\overline{\mathcal{F}}, \Theta)$ is $F$-dlt. Let $A \geq 0$ and $B \geq 0$ be $\mathbb{Q}$-divisors such that $\Delta=A+B$ and $A$ is ample. Assume that $K_{\mathcal{F}}+\Delta$ is nef.

Then $K_{\mathcal{F}}+\Delta$ is semi-ample.

Proof Let $\Gamma=\frac{1}{2} A+B$ and let

$$
\lambda=\min \left\{t \geq 0 \mid K_{\mathcal{F}}+\Gamma+t A \text { is nef }\right\} .
$$


If $\lambda<1 / 2$ then $K_{\mathcal{F}}+\Delta$ is ample and there is nothing to prove. Thus, we may assume that $\lambda=1 / 2$. By Lemma 9.2, there exists a $\left(K_{\mathcal{F}}+\Gamma\right)$-negative extremal ray $R$ such that $\left(K_{\mathcal{F}}+\Delta\right) \cdot \xi=0$ for all $\xi \in R$.

Suppose that $\operatorname{loc}(R) \neq X$. By Theorem 8.4, there exists a morphism $f: X \rightarrow X^{\prime}$ which contracts exactly all the curves in $R$. Let $\mathcal{F}^{\prime}$ be the transformed foliation on $X^{\prime}$ and let $A^{\prime}$ be an ample $\mathbb{Q}$-divisor on $X^{\prime}$ such that $A-f^{*} A^{\prime}$ is also ample. Then there exists a $\mathbb{Q}$-divisor $A^{\prime \prime} \geq 0$ on $X^{\prime}$ and a $\mathbb{Q}$ divisor $B \geq 0$ on $X$ such that $A^{\prime} \sim_{\mathbb{Q}} A^{\prime \prime}$ and if $\Delta^{\prime}:=f^{*} A^{\prime \prime}+B^{\prime}$, then $\left(\mathcal{F}, \Delta^{\prime}\right)$ is $\log$ canonical. Let $\Delta^{\prime \prime}$ be the image of $\Delta^{\prime}$ in $X^{\prime}$. Then $\Delta^{\prime \prime}=A^{\prime \prime}+B^{\prime \prime}$ where $B^{\prime \prime} \geq 0$ and $\left(\mathcal{F}^{\prime}, \Delta^{\prime \prime}\right)$ is lc. Note that $\rho\left(X^{\prime}\right)<\rho(X)$. Lemma 8.5 implies that $X^{\prime}$ is potentially klt.

If $f$ is a flipping contraction then the existence of a small $\mathbb{Q}$-factorialisation $\pi: \overline{X^{\prime}} \rightarrow X^{\prime}$ satisfying the hypotheses of the lemma is an immediate consequence of the existence by such a small $\mathbb{Q}$-factorialisation for $X$. By Lemma 3.33, it follows that $\mathcal{F}^{\prime}$ has non-dicritical singularities. Thus we may replace $(\mathcal{F}, \Delta)$ by $\left(\mathcal{F}^{\prime}, \Delta^{\prime \prime}\right)$ and continue.

Now suppose $f$ is a divisorial contraction. Consider a diagram as in the proof of Lemma 8.3

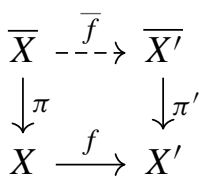

where $\pi: \bar{X} \rightarrow X$ is a small $\mathbb{Q}$-factorialisation satisfying the hypotheses of the lemma, $\bar{f}$ is a $\left(K_{\overline{\mathcal{F}}}+\bar{\Gamma}\right)$-MMP where $K_{\overline{\mathcal{F}}}+\bar{\Gamma}=\pi^{*}\left(K_{\mathcal{F}}+\Gamma\right)$ and $\pi^{\prime}$ is the induced morphism. We claim that $\pi^{\prime}: \overline{X^{\prime}} \rightarrow X^{\prime}$ satisfies the hypotheses of the lemma. It is immediate that $\overline{X^{\prime}}$ is projective and $\mathbb{Q}$-factorial since $\bar{X}$ is and $\pi^{\prime}$ is small. We may choose $\epsilon, \delta>0$ sufficiently small and $\Theta$ on $\bar{X}$ such that $(\overline{\mathcal{F}}, \Theta)$ is F-dlt as guaranteed by our hypotheses and such that $\bar{f}$ is $\left(K_{\overline{\mathcal{F}}}+\Theta-\delta \pi^{*} A\right)$-MMP. Thus, if we let $\Theta^{\prime}=\bar{f}_{*}\left(\Theta-\epsilon \pi^{*} A\right)$ we see that $\left(\overline{\mathcal{F}^{\prime}}, \Theta^{\prime}\right)$ is F-dlt. Observe again, that Lemma 3.33 implies that $\mathcal{F}^{\prime}$ has non-dicritical singularities. We may therefore replace $(\mathcal{F}, \Delta)$ by $\left(\mathcal{F}^{\prime}, \Delta^{\prime \prime}\right)$ and continue. After finitely many steps we obtain the claim.

Now assume that $\operatorname{loc}(R)=X$. Let $H_{R}=K_{\mathcal{F}}+\Delta+A$ be a supporting hyperplane to $R$ where $A$ is an ample divisor and let $v=v\left(H_{R}\right)<3$. Observe that $H_{R}^{3}=0$ and $\left(K_{\mathcal{F}}+\Delta\right) \cdot D_{1} \cdot D_{2}<0$ where $D_{i}=H_{R}$ for $1 \leq i \leq v$ and $D_{i}=A$ otherwise. Then we may apply [41, Corollary 2.28] to see that $X$ is covered by rational curves tangent to $\mathcal{F}$ and spanning $R$.

Let $C$ be a general curve spanning $R$ tangent to $\mathcal{F}$ and let $C^{\prime}$ be the strict transform of $C$ on $\bar{X}$. Notice that $K_{\overline{\mathcal{F}}} \cdot C<0$ and $K_{\bar{X}} \cdot C<0$. We may run a $K_{\bar{X}}$-MMP where each step of the MMP is $K_{\overline{\mathcal{F}}}+\bar{\Delta}$-trivial. Call this 
MMP $\psi: \bar{X} \rightarrow W$ and set $\mathcal{H}=\psi_{*} \overline{\mathcal{F}}$ and $\Gamma=\psi_{*} \bar{\Delta}$. Notice that $(\mathcal{H}, \Gamma)$ has canonical singularities. Observe that it suffices to show that $K_{\mathcal{H}}+\Gamma$ is semi-ample.

Notice that our MMP will terminate with a Mori fibre space $f: W \rightarrow Y$ whose fibres are tangent to $\mathcal{H}$. Notice that, as in the proof of [41, Theorem 8.13], it follows that $f$ is $K_{X}$-negative. Thus, $Y$ is klt. By Lemma 9.1, there is a foliation $\mathcal{G}$ on $Y$, a semi-ample divisor $D$ and $\Theta \geq 0$ such that $(\mathcal{G}, \Theta)$ is lc and

$$
K_{\mathcal{H}}+\Gamma \sim_{\mathbb{Q}} f^{*}\left(K_{\mathcal{G}}+\Theta+D\right) .
$$

Let $G$ be an ample divisor on $Y$ and choose $0<\delta \ll 1$ such that $\Delta-$ $\delta f^{*} G \sim_{\mathbb{Q}} \Delta^{\prime} \geq 0$ and $\left(\mathcal{F}, \Delta^{\prime}\right)$ is lc. Since $K_{\mathcal{F}}+\Delta^{\prime} \sim_{\mathbb{Q}, f} 0$, we may apply Lemma 9.1 again to find $D^{\prime}$ and $\Theta^{\prime} \geq 0$ such that $\Theta+D \sim_{\mathbb{Q}} \Theta^{\prime}+D+$ $D^{\prime}+\delta G \sim_{\mathbb{Q}} \Theta^{\prime \prime} \geq 0$ where $\left(\mathcal{G}, \Theta^{\prime \prime}\right)$ is lc. Replacing $X$ and $(\mathcal{F}, \Delta)$ by $Y$ and $\left(\mathcal{G}, \Theta^{\prime \prime}\right)$ respectively and proceeding as above, we obtain the claim.

Theorem 9.4 Let $X$ be a normal projective threefold and let $\mathcal{F}$ be a co-rank one foliation with non-dicritical singularities. Suppose that $X$ is potentially klt. Let $\Delta$ be a $\mathbb{Q}$-divisor such that $(\mathcal{F}, \Delta)$ is a F-dlt pair. Let $A \geq 0$ and $B \geq 0$ be $\mathbb{Q}$-divisors such that $\Delta=A+B$ and $A$ is ample. Assume that $K_{\mathcal{F}}+\Delta$ is nef.

Then $K_{\mathcal{F}}+\Delta$ is semi-ample.

Proof The Theorem follows immediately from Lemmas 3.29 and 9.3.

\section{Minimal model program with scaling}

The goal of this section is to show the existence of a minimal model for a F-dlt pair $(\mathcal{F}, A+B)$ where $A \geq 0$ is an ample $\mathbb{Q}$-divisor, $B \geq 0$ and such that $K_{\mathcal{F}}+A+B$ has non-negative Kodaira dimension. To this end, we are not able to show termination of flips in general, but we can show that a special sequence of flips terminates. This process is called MMP with scaling. Below, we adopt many of the techniques used in [7].

Let $f: X \rightarrow Y$ be a proper birational map of normal varieties and let $D$ be a $\mathbb{Q}$-divisor on $X$ such that both $D$ and $D^{\prime}:=f_{*} D$ are $\mathbb{Q}$-Cartier. We say that $f$ is $D$-non-positive if for any resolution of indeterminacy $p: W \rightarrow X$ and $q: W \rightarrow Y$, we may write

$$
p^{*} D=q^{*} D^{\prime}+E,
$$

where $E \geq 0$ is $q$-exceptional.

In particular, if $(\mathcal{F}, \Delta)$ is a F-dlt foliation on a normal projective variety $X$, then a sequence of $\left(K_{\mathcal{F}}+\Delta\right)$-flips and divisorial contractions is a $\left(K_{\mathcal{F}}+\right.$ 
$\Delta$ )-non-positive birational map. A minimal model of $(\mathcal{F}, \Delta)$ is a $\left(K_{\mathcal{F}}+\Delta\right)$ non-positive birational map $f: X \rightarrow X^{\prime}$ such that if $\mathcal{F}^{\prime}$ is the transformed foliation on $X^{\prime}$ and $\Delta^{\prime}=f_{*} \Delta$, then

1. $X^{\prime}$ is $\mathbb{Q}$-factorial and klt and $\mathcal{F}^{\prime}$ is non-dicritical,

2. $\left(\mathcal{F}^{\prime}, \Delta^{\prime}\right)$ is F-dlt and $K_{\mathcal{F}^{\prime}}+\Delta^{\prime}$ is nef, and

3. if $E$ is a $f^{-1}$-exceptional divisor on $X^{\prime}$ then $E$ is $\mathcal{F}^{\prime}$-invariant and $a(E, \mathcal{F})=0$.

Lemma 10.1 Let $\mathcal{F}$ be a co-rank one foliation with non-dicritical singularities on a normal $\mathbb{Q}$-factorial projective threefold $X$. Let $\Delta$ be a $\mathbb{Q}$-divisor such that $(\mathcal{F}, \Delta)$ is a F-dlt pair. Let $A \geq 0$ and $B \geq 0$ be $\mathbb{Q}$-divisors such that $\Delta \sim_{\mathbb{Q}} A+B, A$ is ample and $(\mathcal{F}, A+B)$ is $F$-dlt. Assume that $H \geq 0$ is a $\mathbb{Q}$-divisor such that $K_{\mathcal{F}}+\Delta+H$ is nef and

$$
K_{\mathcal{F}}+\Delta \sim_{\mathbb{R}} D+\alpha H,
$$

where $\alpha \geq 0$, and $D \geq 0$ is a $\mathbb{R}$-divisor whose support is a union of lc centres of $(\mathcal{F}, \Delta)$.

Then there exists a birational contraction $f: X \rightarrow Y$ which is a minimal model for $(\mathcal{F}, \Delta)$.

Proof Note that, by Lemma 3.16, $X$ is klt. Let

$$
\lambda=\inf \left\{t>0 \mid K_{\mathcal{F}}+\Delta+t H \text { is nef }\right\} .
$$

If $\lambda=0$, then $K_{\mathcal{F}}+\Delta$ is nef and there is nothing to prove. Otherwise, by Lemma 9.2, there exists a curve $\xi$ in $X$ such that $R=\mathbb{R}_{+}[\xi]$ is an extremal ray of $\overline{N E(X)}$ satisfying:

$$
\left(K_{\mathcal{F}}+\Delta\right) \cdot \xi<0 \quad \text { and } \quad\left(K_{\mathcal{F}}+\Delta+\lambda H\right) \cdot \xi=0 .
$$

Note that, since $(D+\alpha H) \cdot \xi<0, \alpha \geq 0$ and $H \cdot \xi>0$, it follows that $\xi$ is contained in the support of $D$ and, in particular, $\xi$ intersects an lc centre of $(\mathcal{F}, \Delta)$.

By Theorems 6.4 and 6.7, $R$ defines a divisorial contraction or a flip $\phi: X \rightarrow X^{\prime}$ and $X^{\prime}$ is klt and $\mathbb{Q}$-factorial. Let $\mathcal{F}^{\prime}$ be the transformed foliation on $X^{\prime}$ and let $\Delta^{\prime}, H^{\prime}$ and $D^{\prime}$ be the image in $X^{\prime}$ of $\Delta, H$ and $D$ respectively. It follows that $K_{\mathcal{F}^{\prime}}+\Delta^{\prime}+\lambda H^{\prime}$ is nef. By Lemma 3.11, $\left(\mathcal{F}^{\prime}, \Delta^{\prime}\right)$ is F-dlt and by Lemma 3.33, $\mathcal{F}^{\prime}$ is non-dicritical.

By Lemma 3.27, there exist $\mathbb{Q}$-divisors $A^{\prime} \geq 0$ and $B^{\prime} \geq 0$ such that $\Delta^{\prime} \sim_{\mathbb{Q}} A^{\prime}+B^{\prime}, A^{\prime}$ is ample and $\left(\mathcal{F}^{\prime}, A^{\prime}+B^{\prime}\right)$ is F-dlt.

Thus, we may replace $X, \Delta, \mathcal{F}, D, H$ and $\alpha$ by $X^{\prime}, \mathcal{F}^{\prime}, \Delta^{\prime}, D^{\prime}, \lambda H^{\prime}$ and $\alpha / \lambda$ respectively and we proceed as above. Theorem 7.1 implies that, after finitely many steps, we obtain a minimal model of $(\mathcal{F}, \Delta)$. 
Lemma 10.2 Let $\mathcal{F}$ be a non-dicritical co-rank one foliation on a smooth projective threefold $X$. Let $\Delta=A+B$ be a $\mathbb{Q}$-divisor such that $(\mathcal{F}, \Delta)$ is a $F$-dlt pair, $A \geq 0$ is an ample $\mathbb{Q}$-divisor and $B \geq 0$. Assume that there exists $a \mathbb{Q}$-divisor $D \geq 0$ such that

1. $K_{\mathcal{F}}+\Delta \sim_{\mathbb{Q}} D$,

2. $(\mathcal{F}, \Delta+D)$ is $\log$ smooth, and

3. any component of $D$ is either semi-ample or it is contained in the stable base locus of $D$.

Then there exists a birational contraction $f: X \rightarrow Y$ which is a minimal model for $(\mathcal{F}, \Delta)$.

Proof We may write $D=D_{1}+D_{2}$ where $D_{1}, D_{2} \geq 0$ and the components of $D_{1}$ are exactly the components of $D$ which are lc centres of $(\mathcal{F}, \Delta)$. Note that, in particular, $D_{1}$ contains all the components of $D$ which are $\mathcal{F}$-invariant. Let $k$ be the number of components of $D_{2}$. We proceed by induction on $k$.

If $k=0$, then $D_{2}=0$ and the support of $D$ is a union of lc centres of $(\mathcal{F}, \Delta)$. Let $H$ be a sufficiently ample $\mathbb{Q}$-divisor such that $K_{\mathcal{F}}+\Delta+H$ is ample. Then Lemma 10.1 implies that there exists a birational contraction $f: X \rightarrow Y$ which is a minimal model for $(\mathcal{F}, \Delta)$.

We now assume that $k>0$. Let

$$
\lambda=\sup \left\{t \geq 0 \mid\left(\mathcal{F}, \Delta+t D_{2}\right) \text { is } F \text {-dlt }\right\} .
$$

By Item (2), it follows that $\lambda>0$ and $\left(\mathcal{F}, \Delta+\lambda D_{2}\right)$ is F-dlt. Note that $\lambda \in \mathbb{Q}$. Moreover, we have

$$
K_{\mathcal{F}}+\Delta+\lambda D_{2} \sim_{\mathbb{Q}} D+\lambda D_{2}
$$

By induction, it follows that $\left(\mathcal{F}, \Delta+\lambda D_{2}\right)$ admits a minimal model $X \rightarrow X^{\prime}$, which is a birational contraction. Let $\mathcal{F}^{\prime}$ be the transformed foliation on $X^{\prime}$ and let $\Delta^{\prime}, D^{\prime}, D_{1}^{\prime}$ and $D_{2}^{\prime}$ be the image of $\Delta, D, D_{1}$ and $D_{2}$ on $X^{\prime}$ respectively. Let $H^{\prime}=\lambda D_{2}^{\prime}$. Then $K_{\mathcal{F}^{\prime}}+\Delta^{\prime}+H^{\prime}$ is nef and

$$
K_{\mathcal{F}^{\prime}}+\Delta^{\prime} \sim_{\mathbb{Q}} D_{1}^{\prime}+\frac{1}{\lambda} H^{\prime}
$$

Thus, Lemma 10.1 implies that there exists a birational contraction $X^{\prime} \rightarrow-Y$ which is a minimal model for $\left(\mathcal{F}^{\prime}, \Delta^{\prime}\right)$.

Let $f: X \rightarrow Y$ be the induced map. Note that $f$ is a birational contraction. In order to show that $f: X \rightarrow Y$ is a minimal model for $(\mathcal{F}, \Delta)$, it is enough to show that $f$ is $\left(K_{\mathcal{F}}+\Delta\right)$-non-positive. Let $\mathcal{G}$ be the transformed foliation on $Y$ and let $\Gamma=f_{*} \Delta$. By Lemma 3.27, there exists $\mathbb{Q}$-divisors $A^{\prime} \geq 0$ and 
$B^{\prime} \geq 0$ such that $\Gamma \sim_{\mathbb{Q}} A^{\prime}+B^{\prime}, A^{\prime}$ is ample and $\left(\mathcal{F}^{\prime}, A^{\prime}+B^{\prime}\right)$ is F-dlt. Thus, Theorem 9.4 implies that $K_{\mathcal{G}}+\Gamma$ is semi-ample.

Let $p: W \rightarrow X$ and $q: W \rightarrow Y$ be a resolution of indeterminacy of $f$. Then, we may write

$$
p^{*}\left(K_{\mathcal{F}}+\Delta\right)+F=q^{*}\left(K_{\mathcal{G}}+\Gamma\right)+E,
$$

where $E, F \geq 0$ are $q$-exceptional $\mathbb{Q}$-divisors without any common component. Since $K_{\mathcal{G}}+\Gamma$ is semi-ample, it follows that the stable base locus of $q^{*}\left(K_{\mathcal{G}}+\Gamma\right)+E$ coincides with the support of $E$. Let us assume that $F \neq 0$. Then, we claim that there exists a component $S$ of $F$ which is contained in the stable base locus of $p^{*} D+F$. Indeed either there exists a component $S$ of $F$ which is $p$-exceptional and the claim follows immediately or the image $T$ of a component $S$ of $F$ in $X$ is $f$-exceptional. In particular, $T$ is contained in the support of $D$ and, by Item 3, $T$ is contained in the stable base locus of $D$. It follows that $S$ is contained in the stable base locus of $p^{*} D+F$. Thus, $S$ is a component of $E$, a contradiction. It follows that $F=0$ and, in particular, $f$ is $\left(K_{\mathcal{F}}+\Delta\right)$-non-positive. Thus, $f: X \rightarrow Y$ is a minimal model for $(\mathcal{F}, \Delta)$.

Theorem 10.3 Let $\mathcal{F}$ be a co-rank one foliation with non-dicritical singularities on a $\mathbb{Q}$-factorial projective threefold X. Let $\Delta=A+B$ be a $\mathbb{Q}$-divisor such that $(\mathcal{F}, \Delta)$ is a $F$-dlt pair, $A \geq 0$ is an ample $\mathbb{Q}$-divisor and $B \geq 0$. Assume that there exists a $\mathbb{Q}$-divisor $D \geq 0$ such that $K_{\mathcal{F}}+\Delta \sim_{\mathbb{Q}} D$.

Then $(\mathcal{F}, \Delta)$ admits a minimal model.

Proof By Lemma 3.26, after possibly replacing $A$ by a $\mathbb{Q}$-equivalent divisor, we may assume that $\lfloor\Delta\rfloor=0$ and that, for any exceptional divisor $E$ over $X$, if $a(E, \mathcal{F}, \Delta)=-\epsilon(E)$ then $E$ is invariant and $a(E, \mathcal{F})=a(E, \mathcal{F}, \Delta)=0$.

By [7, Proposition 3.5.4], we may find a positive integer $m$ and $\mathbb{Q}$-divisors $P \geq 0$ and $N \geq 0$ such that $P+N \sim_{\mathbb{Q}} D$ and any component of $N$ is contained in the stable base locus of $P+N$, whilst every component $\Sigma$ of $P$ is such that $m \Sigma$ is mobile. Let $\pi: Z \rightarrow X$ be a foliated log resolution of $(\mathcal{F}, \Delta+P+N)$ which also resolves the base locus of $|m \Sigma|$ for any component $\Sigma$ of $P$. Let $\mathcal{G}$ be the transformed foliation on $Z$. We may write

$$
K_{\mathcal{G}}+\Delta_{Z}=\pi^{*}\left(K_{\mathcal{F}}+\Delta\right)+F
$$

for some $\mathbb{Q}$-divisors $\Delta_{Z}, F \geq 0$ without common components. Let $C \geq 0$ be a $\pi$-exceptional $\mathbb{Q}$-divisor on $Z$ such that $\pi^{*} A-C$ is ample. Notice that $\pi^{*} A-t C$ is ample for any $0<t<1$.

Thus, there exist $\delta, \epsilon>0$ and a $\mathbb{Q}$-divisor $\Gamma \sim_{\mathbb{Q}} \Delta_{Z}-\delta C+\epsilon \sum E_{i}$ where the sum is taken over all the non-invariant $\pi$-exceptional divisors and such that 
1. $\Gamma=A^{\prime}+B^{\prime}$ where $A^{\prime} \geq 0$ is an ample $\mathbb{Q}$-divsor and $B^{\prime} \geq 0$,

2. $(\mathcal{G}, \Gamma)$ is F-dlt,

3. we may write

$$
K_{\mathcal{G}}+\Gamma \sim_{\mathbb{Q}} \pi^{*}\left(K_{\mathcal{F}}+\Delta\right)+F^{\prime}
$$

where $F^{\prime} \geq 0$ is a $\pi$-exceptional $\mathbb{Q}$-divisor, whose support contains every exceptional divisor $E$ of $\pi$ such that $a(E, \mathcal{F})>-\epsilon(E)$,

4. there exists an effective divisor $D^{\prime} \sim_{\mathbb{Q}} K_{\mathcal{G}}+\Gamma$ such that any component of $D^{\prime}$ is either semi-ample or it is contained in the stable base locus of $D^{\prime}$, and

5. $\left(\mathcal{G}, \Gamma+D^{\prime}\right)$ is a $\log$ smooth foliation.

Lemma 10.2 implies that $(\mathcal{G}, \Gamma)$ admits a minimal model $g: Z \rightarrow Y$, which is a birational contraction. We want to show that the induced map $f: X \rightarrow Y$ is a minimal model of $(\mathcal{F}, \Delta)$. Let $p: W \rightarrow Z$ and $q: W \rightarrow Y$ be proper birational morphisms that resolve the indeterminacy locus of $g$. Let $r: W \rightarrow X$ be the induced morphism. Since $g$ is $\left(K_{\mathcal{G}}+\Gamma\right)$-non-positive, we may write

$$
p^{*}\left(K_{\mathcal{G}}+\Gamma\right)=q^{*}\left(K_{\mathcal{F}^{\prime}}+\Gamma^{\prime}\right)+G
$$

where $\mathcal{F}^{\prime}$ is the transformed foliation on $Y, \Gamma^{\prime}=g_{*} \Gamma$ and $G \geq 0$ is $q$ exceptional. On the other hand, we also have

$$
p^{*}\left(K_{\mathcal{G}}+\Gamma\right) \sim_{\mathbb{Q}} r^{*}\left(K_{\mathcal{F}}+\Delta\right)+p^{*} F^{\prime} .
$$

Since $K_{\mathcal{F}^{\prime}}+\Gamma^{\prime}$ is nef, the negativity lemma implies that $G \geq p^{*} F^{\prime}$. In particular, the support of $G$ contains every exceptional divisor $E$ of $\pi$ such that $a(E, \mathcal{F})>-\epsilon(E)$. Thus, if $E^{\prime}$ is a $f^{-1}$-exceptional divisor on $Y$ then $E^{\prime}$ is invariant and $a\left(E^{\prime}, \mathcal{F}\right)=-\epsilon(E)=0$. Moreover, it follows that $f$ is $\left(K_{\mathcal{F}}+\Delta\right)$-non-positive. Thus, the claim follows.

\section{Existence of F-terminalisations}

Theorem 11.1 (Existence of F-terminalisations) Let $\mathcal{F}$ be a co-rank one foliation on a normal variety $X$ of dimension $\leq 3$. Let $(\mathcal{F}, \Delta)$ be a foliated pair with $\Delta \geq 0$.

Then there exists a birational morphism $\pi: Y \rightarrow X$ such that

1. if $\mathcal{G}$ is the transformed foliation and $\Delta_{Y}=\pi_{*}^{-1} \Delta$, then $\left(\mathcal{G}, \Delta_{Y}\right)$ is F-dlt, canonical and terminal along Sing $Y$,

2. $Y$ is klt and $\mathbb{Q}$-factorial and

3. $K_{\mathcal{G}}+\Delta_{Y}+E=\pi^{*}\left(K_{\mathcal{F}}+\Delta\right)$ where $E \geq 0$. 
Proof We assume that $\operatorname{dim} X=3$. The case where $\operatorname{dim} X=2$ is similar. Let $\mu: W \rightarrow X$ be a foliated log resolution of $\mathcal{F}$, let $\mathcal{H}$ be the pulled back foliation on $W$ and let $\Delta^{\prime}=\mu_{*}^{-1} \Delta$. Let $A$ be an ample Cartier divisor on $X$ and let $C \geq 0$ be a $\mu$-exceptional $\mathbb{Q}$-divisor on $W$ such that $\mu^{*} A-C$ is ample. Let $0<\delta \ll 1$ such that if $\Gamma=\mu^{*} A-\delta C$ then

$$
K_{\mathcal{H}}+\Delta^{\prime}+\Gamma+G_{1}=\mu^{*}\left(K_{\mathcal{F}}+\Delta+A\right)+G_{2},
$$

where $G_{1}, G_{2} \geq 0$ are $\mu$-exceptional $\mathbb{Q}$-divisors without any common component and the support of $G_{2}$ contains all the $\mu$-exceptional divisors with discrepancy greater than zero with respect to $(\mathcal{F}, \Delta)$.

For $0<\epsilon \ll 1$ we have that $\mu^{*} A-\delta C+\epsilon\left\lfloor\Delta^{\prime}\right\rfloor$ is ample. Thus, by Lemma 3.24 and Remark 2.13 we may find $0 \leq A^{\prime} \sim_{\mathbb{Q}} \Gamma+\epsilon\left\lfloor\Delta^{\prime}\right\rfloor$ such that $\left(\mathcal{H}, \Delta^{\prime \prime}+A^{\prime}\right)$ is F-dlt where $\Delta^{\prime \prime}:=\Delta^{\prime}-\epsilon\left\lfloor\Delta^{\prime}\right\rfloor$. We claim that if $n \geq 6$ then any $\left(K_{\mathcal{H}}+\Delta^{\prime \prime}+A^{\prime}+n \mu^{*} A\right)$-negative extremal ray is generated by a curve which is contracted by $\mu$. Indeed let $C$ be a curve spanning a $\left(K_{\mathcal{H}}+\Delta^{\prime \prime}+A^{\prime}\right)$ negative extremal ray and suppose that $\mu_{*} C \neq 0$. On one hand, Theorem 3.31 implies that $-\left(K_{\mathcal{H}}+\Delta^{\prime \prime}+A^{\prime}\right) \cdot C \leq 6$, on the other hand $n \mu^{*} A \cdot C \geq 6$ and so $\left(K_{\mathcal{H}}+\Delta^{\prime \prime}+A^{\prime}+n \mu^{*} A\right) \cdot C \geq 0$, proving our claim.

By choosing $n$ sufficiently large, we may also assume that there exists a $\mathbb{Q}$ divisor $D \geq 0$ on $W$ such that $D \sim_{\mathbb{Q}} K_{\mathcal{H}}+\Delta^{\prime \prime}+A^{\prime}+n \mu^{*} A$. By Lemma 3.24, Remark 2.13 and Corollary 3.25 we may find $0 \leq A^{\prime \prime} \sim_{\mathbb{Q}} A^{\prime}+n \mu^{*} A$ such that $\left(\mathcal{H}, \Delta^{\prime \prime}+A^{\prime \prime}\right)$ is F-dlt and canonical. By Theorem $10.3, K_{\mathcal{H}}+\Delta^{\prime \prime}+A^{\prime \prime}$ admits a minimal model $f: W \rightarrow Y$.

We claim that each step in this MMP will be an MMP over $X$. Indeed, as observed above, the first step of this MMP must contract only $\mu$-exceptional curves, and so this first step is a step over $X$. Let $W^{\prime} \rightarrow W^{\prime \prime}$ be an intermediate step of this MMP. By induction, we have a morphism $\mu^{\prime}: W^{\prime} \rightarrow X$ and, as observed earlier, Theorem 3.31 implies that if $f^{\prime}: W \rightarrow W^{\prime}$ is the induced map then every $\left(K_{f_{*}^{\prime} \mathcal{H}}+f_{*}^{\prime} \Delta^{\prime \prime}+f_{*}^{\prime} A^{\prime \prime}\right)$-negative extremal ray is spanned by a curve $C$ such that $\left(\mu^{\prime}\right)^{*} A \cdot C=0$, and so this step of the MMP will again be a step over $X$. Let $\pi: Y \rightarrow X$ be the induced morphism.

Note that, Lemma 2.7 implies that if $\mathcal{G}$ is the transformed foliation and $\Delta_{Y}=f_{*} \Delta^{\prime}=\pi_{*}^{-1} \Delta$, then $\mathcal{G}$ is F-dlt and canonical and, moreover it is terminal along Sing $Y$. Moreover, by definition of minimal model, we have that $Y$ is klt and $\mathbb{Q}$-factorial. Finally, $f_{*}\left(G_{2}-G_{1}\right)$ is nef over $X$ and $\pi$ exceptional and so the negativity lemma applies to show that $f_{*} G_{2}=0$. Thus, if $E:=\pi^{*}\left(K_{\mathcal{F}}+\Delta\right)-\left(K_{\mathcal{G}}+\Delta_{Y}\right)$ then $E \geq 0$.

Definition 11.2 We call a modification $\pi: Y \rightarrow X$ as in Theorem 11.1 an F-terminalisation for the foliated pair $(\mathcal{F}, \Delta)$.

Theorem 11.3 Let $(\mathcal{F}, \Delta)$ be a foliated pair on a projective threefold $X$. Assume that $\mathcal{F}$ is a co-rank one foliation and either 
1. $(\mathcal{F}, \Delta)$ is F-dlt or

2. $(\mathcal{F}, \Delta)$ is canonical.

Then $\mathcal{F}$ has non-dicritical singularities.

Furthermore, if $(\mathcal{F}, \Delta)$ is $F$-dlt and $K_{X}$ is $\mathbb{Q}$-Cartier then $X$ is klt.

Proof We will only prove the case where $(\mathcal{F}, \Delta)$ is F-dlt. The other one may be handled in a similar manner. Let $\mu: W \rightarrow X$ be a foliated $\log$ resolution of $(\mathcal{F}, \Delta)$ which only extracts divisors $E$ of discrepancy $-\epsilon(E)$ and let $\mathcal{H}$ be the pulled back foliation on $W$. Our result follows if we can show $\mu^{-1}(P)$ is tangent to $\mathcal{H}$ for all $P \in X$. So suppose for sake of contradiction that there is some $P$ such that $\mu^{-1}(P)$ is not tangent to $\mathcal{H}$ and let $C \subset \mu^{-1}(P)$ be a general curve transverse to the foliation.

Write $K_{\mathcal{H}}+\Gamma=\mu^{*}\left(K_{\mathcal{F}}+\Delta\right)+E$ where $E \geq 0$ is $\mu$-exceptional, $\Gamma \geq 0$ and $\mu_{*} \Gamma=\Delta$, so that $E$ and $\Gamma$ do not have any common component. Observe that $\left\lfloor\Gamma-\mu_{*}^{-1} \Delta\right\rfloor=0$. Let $A \geq 0$ be an ample divisor on $X$ and let $G \geq 0$ be a $\mu$-exceptional $\mathbb{Q}$-divisor on $W$ such that $\mu^{*} A-G$ is ample. Let $F$ be the sum of all the $\mu$-exceptional non-invariant divisors. There exist sufficiently small $\epsilon, \delta>0$ such that if $\Theta=\mu^{*} A-\delta G+\Gamma+\epsilon F$, then we may write

$$
K_{\mathcal{H}}+\Theta+E_{1}=\mu^{*}\left(K_{\mathcal{F}}+\Delta+A\right)+E_{2},
$$

where $E_{1}, E_{2} \geq 0$ are $\mu$-exceptional $\mathbb{Q}$-divisors without common components and such that the support of $E_{2}$ contains all the $\mu$-exceptional non-invariant divisors.

As in the proof of Theorem 11.1, by Theorem 10.3, we may run a $\left(K_{\mathcal{H}}+\Theta+\right.$ $n \mu^{*} A$ )-MMP $\phi: W \rightarrow Y$, where $n$ is sufficiently large so that the induced map $v: Y \rightarrow X$ is a proper morphism. Let $\mathcal{G}$ be the transformed foliation on $Y$. Notice that, the negativity lemma implies that $\phi_{*} E_{2}=0$ and, in particular, $\phi$ contracts all the non-invariant $\mu$-exceptional divisors. Moreover, we have

$$
E_{2}-E_{1}=E-\delta G+\epsilon F .
$$

Thus, if $\delta$ is sufficiently small, then the support of $E$ is contained in the support of $E_{2}$ and therefore $\phi_{*} E=0$. It follows that

$$
K_{\mathcal{G}}+\phi_{*} \Gamma=K_{\mathcal{G}}+v_{*}^{-1} \Delta=v^{*}\left(K_{\mathcal{F}}+\Delta\right)
$$

and that every $\nu$-exceptional divisor is $\mathcal{G}$-invariant. Since $C$ is transverse to the foliation we have $0 \neq \phi_{*} C=: C^{\prime}$ is also transverse to the foliation and so is not contained in any $v$-exceptional divisor. Let $A_{1}$ and $A_{2}$ be two distinct effective Cartier divisors containing $v\left(C^{\prime}\right)=P$ and write $v^{*} A_{i}=v_{*}^{-1} A_{i}+B_{i}$ where $B_{i} \geq 0$ is $v$-exceptional. On one hand we know that $B_{i} \cdot C^{\prime}>0$, on 
the other hand we know that $v^{*} A_{i} \cdot C^{\prime}=0$ and so $v_{*}^{-1} A_{i} \cdot C^{\prime}<0$. Let $D=v_{*}^{-1} A_{1}+v_{*}^{-1} A_{2}$ and let

$\lambda=\sup \left\{t \geq 0 \mid\left(\mathcal{G}, v_{*}^{-1} \Delta+t D\right)\right.$ is $\log$ canonical at the generic point of $\left.C^{\prime}\right\}$.

We claim that $\lambda>0$. Indeed, suppose that $\lambda=0$. In this case $C^{\prime}$ is an lc centre of $\left(\mathcal{G}, v_{*}^{-1} \Delta\right)$, which in turn implies that $v\left(C^{\prime}\right)=P$ is an lc centre of $(\mathcal{F}, \Delta)$. By Lemma 3.8, it follows that $\mathcal{F}$ has simple singularities near $P$, and so, by Remark 2.13, we have that $\mathcal{F}$ is non-dicritical near $P$, a contradiction.

Thus, by the definition of $\lambda$, it follows that $C^{\prime}$ is an lc centre of $\left(\mathcal{G}, v_{*}^{-1} \Delta+\right.$ $\lambda D)$. Moreover, we have $\left(K_{\mathcal{G}}+v_{*}^{-1} \Delta+\lambda D\right) \cdot C^{\prime}<0$. This however is a contradiction of foliation subadjunction, [41, Theorem 4.5] which implies that $\left(K_{\mathcal{G}}+v_{*}^{-1} \Delta+\lambda D\right) \cdot C^{\prime} \geq 0$.

To see our final claim, since $(\mathcal{F}, \Delta)$ is F-dlt, we may find a log resolution $\mu: W \rightarrow X$ only extracting divisors $E$ of discrepancy $>-\epsilon(E)$. We run a $\left(K_{\mathcal{H}}+\mu_{*}^{-1} \Delta+F\right)$-MMP over $X$, where $F$ is the sum of all the $\mu$-exceptional non-invariant divisors. Note that this MMP terminates by Corollary 7.10. Let $\phi: W \rightarrow Y$ be the output of this MMP, with induced morphism $v: Y \rightarrow X$. Observe that $Y$ is klt. By the negativity lemma, we know that $v$ is small and so $K_{Y}=v^{*} K_{X}$ which implies that $X$ is klt.

Remark 11.4 Theorem 11.3 shows that the hypothesis of non-dicriticality in the cone theorem (and in the above results) is superfluous. When $X$ is smooth this result follows from [29, Proposition 3.11].

Proof of Theorems 1.1 and 1.2 Note that Theorem 1.1 (resp. Theorem 1.2) follows directly from Theorems 11.3 and 6.4 (resp. Theorem 10.3).

\section{Abundance for $c_{1}\left(K_{\mathcal{F}}+\Delta\right)=0$}

The goal of this section is to prove the following:

Theorem 12.1 Let $X$ be a projective threefold and $\mathcal{F}$ be a co-rank one foliation. Let $(\mathcal{F}, \Delta)$ be a foliated pair with log canonical singularities where $\Delta \geq 0$ is a $\mathbb{Q}$-divisor such that $c_{1}\left(K_{\mathcal{F}}+\Delta\right)=0$.

Then $\kappa\left(K_{\mathcal{F}}+\Delta\right)=0$.

As we mentioned in the Introduction, the result above is a consequence of [29, Theorem 2] in the case of foliations with canonical singularities defined on a smooth projective variety. See also [16, Theorem 1.3] for results in this direction on singular varieties, as well as the recent preprint [15], which achieves the main result of Sect. 12.2 in all dimensions. 


\section{1 $\Delta \neq 0$ or $\mathcal{F}$ is $\log$ canonical but not canonical}

Lemma 12.2 Suppose $X$ is a klt projective surface and $\mathcal{F}$ is a rank one foliation on $X$. Suppose that $(\mathcal{F}, \Delta)$ is lc where $\Delta \geq 0$ is a $\mathbb{Q}$-divisor and $c_{1}\left(K_{\mathcal{F}}+\Delta\right)=0$.

Then $\kappa\left(K_{\mathcal{F}}+\Delta\right)=0$.

Proof Without loss of generality we may replace $(\mathcal{F}, \Delta)$ by an F-dlt modification. In particular, we may assume that $\mathcal{F}$ has canonical singularities.

If $\Delta=0$, then our claim follows from [31, Lemma IV.3.1].

If $\Delta \neq 0$, then $K_{\mathcal{F}}$ is not pseudo-effective. Running an MMP for $K_{\mathcal{F}}$ with scaling of some ample divisor, and replacing $\mathcal{F}$ by this output we may assume that we have a $\mathbb{P}^{1}$-fibration $f: X \rightarrow C$ such that $\mathcal{F}$ is induced by the fibration.

By Lemma 9.1 we see that $K_{\mathcal{F}}+\Delta \sim_{\mathbb{Q}} f^{*} \Theta$ where $\Theta \geq 0$ and our result is proven.

Lemma 12.3 Let $X$ be a normal projective threefold and $\mathcal{F}$ be a co-rank one foliation on $X$. Suppose that $\mathcal{F}$ is algebraically integrable, $(\mathcal{F}, \Delta)$ is $\log$ canonical where $\Delta \geq 0$ is a $\mathbb{Q}$-divisor and $c_{1}\left(K_{\mathcal{F}}+\Delta\right)=0$.

Then $\kappa\left(K_{\mathcal{F}}+\Delta\right)=0$.

Proof By Theorem 8.1 we may replace $(\mathcal{F}, \Delta)$ by an F-dlt modification and so we may assume without loss of generality that $X$ is $\mathbb{Q}$-factorial and klt.

By assumption $\mathcal{F}$ admits a meromorphic first integral $f: X \rightarrow C$ where $C$ is a smooth proper curve. Let $\mu: X^{\prime} \rightarrow X$ be a resolution of indeterminacies of $f$ and let $f^{\prime}: X^{\prime} \rightarrow C$ be the resolved map. Observe that $f^{\prime}$ is a holomorphic first integral of $\mathcal{F}^{\prime}$, the pull back of $\mathcal{F}$ on $X^{\prime}$.

As $(\mathcal{F}, \Delta)$ is F-dlt it follows that $\mathcal{F}$ has non-dicritical singularities by Theorem 11.3, and so if $p \in X$ then $\mu^{-1}(p)$ is tangent to $\mathcal{F}^{\prime}$. Since $f^{\prime}$ is a holomorphic first integral of $\mathcal{F}^{\prime}$ this implies that $f^{\prime}\left(\mu^{-1}(p)\right)$ is a single point and so $f^{\prime}$ contracts every fibre of $\mu$. The rigidity lemma then implies that in fact $f: X \rightarrow C$ is a morphism.

We apply Lemma 9.1 to write $K_{\mathcal{F}}+\Delta \sim_{\mathbb{Q}} f^{*} \Theta$ for some $\mathbb{Q}$-divisor $\Theta \geq 0$ and we can conclude.

Proposition 12.4 Let $X$ be a normal projective threefold and $\mathcal{F}$ be a co-rank one foliation. Let $(\mathcal{F}, \Delta)$ be a foliated pair with log canonical singularities and $\Delta \geq 0$ is a $\mathbb{Q}$-divisor. Suppose that $c_{1}\left(K_{\mathcal{F}}+\Delta\right)=0$ and that either $\Delta \neq 0$ or $\mathcal{F}$ is $\log$ canonical but not canonical.

Then $\kappa\left(K_{\mathcal{F}}+\Delta\right)=0$.

Proof By Theorem 11.1, we may replace $(\mathcal{F}, \Delta)$ with a F-terminalisation, so we may assume without loss of generality that $(\mathcal{F}, \Delta)$ is F-dlt and canonical with $\Delta \neq 0$ and that $X$ is $\mathbb{Q}$-factorial and klt. 
In this case, $K_{\mathcal{F}}$ is not pseudo-effective and hence it is uniruled by Lemma 2.21. So there exists a diagram

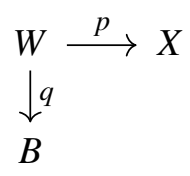

where $q: W \rightarrow B$ parametrizes a dominant family of rational curves tangent to $\mathcal{F}$.

We may obviously assume that $\operatorname{dim}(B)=2$. Let $G$ be an ample divisor on $B$. Let $b \in B$ be general and let $W_{b}$ be the fibre over $b$. There are two cases, either

1. $p\left(W_{b}\right) \cap p\left(W_{b^{\prime}}\right) \neq \emptyset$ for infinitely many $b^{\prime}$ or

2. $p\left(W_{b}\right) \cap p\left(W_{b^{\prime}}\right)=\emptyset$ for $b \neq b^{\prime}$.

Suppose that we are in case (1), and let $Z_{b} \subset B$ be the closed subset of $B$ parametrizing those points $b^{\prime}$ with $p\left(W_{b^{\prime}}\right) \cap p\left(W_{b}\right) \neq \emptyset$. Set $S=p\left(q^{-1}\left(Z_{b}\right)\right)$. Observe that $\mathcal{F}$ has non-dicritical singularities by Theorem 11.3. We claim that $S$ is $\mathcal{F}$-invariant. Supposing the claim we see that a general leaf of $\mathcal{F}$ is algebraic and we may conclude by Lemma 12.3. To see the claim, without loss of generality we may assume that $p\left(W_{b}\right)$ and $p\left(W_{b^{\prime}}\right)$ all pass through the same point $x \in X$. Let $m: X^{\prime} \rightarrow X$ be a resolution of singularities of $X$ such that $m^{-1}(x)$ is a divisor, let $S^{\prime}=m_{*}^{-1} S$ and let $\Sigma_{b}$ and $\Sigma_{b^{\prime}}$ be the strict transforms of $p\left(W_{b}\right)$ and $p\left(W_{b^{\prime}}\right)$ respectively. Observe that $\Sigma_{b^{\prime}} \cap m^{-1}(x) \subset \operatorname{Sing} \mathcal{F}^{\prime}$ where $\mathcal{F}^{\prime}$ is the pulled back foliation. Thus, $S^{\prime}$ is covered by curves tangent to $\mathcal{F}^{\prime}$ which pass through Sing $\mathcal{F}^{\prime}$ which by [12, Théorème 4] and the fact that $\mathcal{F}^{\prime}$ is non-dicritical implies that $S^{\prime}$ must be $\mathcal{F}^{\prime}$-invariant, as required.

So suppose we are in case (2). Note that the rational map $q \circ p^{-1}: X \rightarrow B$ is almost proper, and in particular, we see that

$$
p\left(W_{b}\right) \cdot p_{*} q^{*} G=0
$$

where $G$ is an ample Cartier divsior on $B$. Let $A$ be an ample divisor on $X$. Given a sufficiently large positive integer $m$, we may run a $K_{X}$-MMP with scaling of $A+p_{*} q^{*}(m G)$ Denote this MMP by $\phi: X \rightarrow X^{\prime}$, and let $\left(\mathcal{F}^{\prime}, \Delta^{\prime}\right)$ be the transformed foliated pair on $X^{\prime}$. Each step of this MMP is $\left(K_{\mathcal{F}}+\Delta\right)$-trivial and so we see that $\left(\mathcal{F}^{\prime}, \Delta^{\prime}\right)$ is lc, $c_{1}\left(K_{\mathcal{F}^{\prime}}+\Delta^{\prime}\right)=0$ and that $\kappa\left(K_{\mathcal{F}^{\prime}}+\Delta^{\prime}\right)=0$ implies that $\kappa\left(K_{\mathcal{F}}+\Delta\right)=0$.

Observe that $K_{X} \cdot p\left(W_{b}\right)<0$. If we choose $m$ larger than $6 m^{\prime}$ where $m^{\prime}$ is the Cartier index of $p_{*} q^{*} G$ then, arguing as in the proof of Theorem 11.1, each step of this MMP will be $p_{*} q^{*} m G$ trivial and so this MMP must terminate in a Mori fibre space $f: X^{\prime} \rightarrow S$ such that $S$ is a klt surface and $f$ contracts the 
strict transform of $p\left(W_{b}\right)$. Thus, the fibration $f$ is tangent to $\mathcal{F}^{\prime}$. By Lemma 9.1, there is a foliation $\mathcal{G}$ on $S$ so that $\mathcal{F}^{\prime}=f^{-1} \mathcal{G}$, a pseudo-effective divisor $D$ and divisor $\Theta \geq 0$ such that $K_{\mathcal{F}^{\prime}}+\Delta^{\prime} \sim_{\mathbb{Q}} f^{*}\left(K_{\mathcal{G}}+\Theta+D\right)$ and $(\mathcal{G}, \Theta)$ is lc.

Suppose for sake of contradiction that $D \neq 0$. Since $c_{1}\left(K_{\mathcal{G}}+\Theta\right)=-D$ it follows that $\mathcal{G}$ is uniruled by Lemma 2.21, in particular, $\mathcal{G}$ is algebraically integrable which implies the same holds for $\mathcal{F}$. Since $f$ is $K_{X^{\prime}}$-negative, we see that, in addition, $S$ is klt. Thus, we can apply Lemma 12.2 to conclude that $K_{\mathcal{G}}+\Theta$, and hence $K_{\mathcal{F}^{\prime}}+\Delta^{\prime}$, is torsion.

\section{2 $\Delta=0$ and $\mathcal{F}$ is canonical}

In this section $\mathcal{F}$ is a co-rank one foliation on a normal projective threefold with $c_{1}\left(K_{\mathcal{F}}\right)=0$. Suppose that $\mathcal{F}$ has canonical singularities.

Lemma 12.5 We may freely replace $\mathcal{F}$ by an $F$-terminalisation. Thus we may assume that $X$ is klt and $\mathbb{Q}$-factorial and $\mathcal{F}$ is terminal along Sing $X$. In particular, Sing $X$ is tangent to $\mathcal{F}$.

Proof Let $\pi: Y \rightarrow X$ be an F-terminalisation, whose existence is guaranteed by Theorem 11.1 and let $\mathcal{G}$ be the pulled back foliation on $Y$. By definition, $K_{\mathcal{G}}+F=\pi^{*} K_{\mathcal{F}}$ where $F \geq 0$. On the other hand, since $\mathcal{F}$ is canonical, $K_{\mathcal{G}}=\pi^{*} K_{\mathcal{F}}+E$ where $E \geq 0$. Thus $E=F=0$ and so $c_{1}\left(K_{\mathcal{G}}\right)=0$. Furthermore, if $K_{\mathcal{G}}$ is torsion then so is $K_{\mathcal{F}}$. The last claim follows from [41, Lemma 8.6] together with Theorem 11.3.

Lemma 12.6 Let $\phi: X \rightarrow X^{\prime}$ be a sequence of steps of a $K_{X}-M M P$ such that $\phi$ is birational and let $\mathcal{F}^{\prime}$ be the transformed foliation on $X^{\prime}$. Then

1. $c_{1}\left(K_{\mathcal{F}^{\prime}}\right)=0$;

2. $X^{\prime}$ has klt and $\mathbb{Q}$-factorial singularities,

3. $\mathcal{F}^{\prime}$ has canonical singularities, and

4. Sing $X^{\prime}$ is tangent to $\mathcal{F}^{\prime}$.

Moreover, if $K_{\mathcal{F}^{\prime}}$ is torsion then so is $K_{\mathcal{F}}$.

Proof Each step of the MMP is $K_{\mathcal{F}}$-trivial so we see that $\mathcal{F}^{\prime}$ has canonical singularities and $c_{1}\left(K_{\mathcal{F}^{\prime}}\right)=0$. Furthermore, we see that $K_{\mathcal{F}}=\phi^{*} K_{\mathcal{F}^{\prime}}$ and so if $K_{\mathcal{F}^{\prime}}$ is torsion then so is $K_{\mathcal{F}}$.

Since $X$ has klt and $\mathbb{Q}$-factorial singularities it follows that $X^{\prime}$ has klt and $\mathbb{Q}$-factorial singularities.

By Theorem 11.3 at each step of the MMP the transformed foliation has non-dicritical singularities and so we see that only curves tangent to $\mathcal{F}$ are contracted by the MMP. In particular, we see that the flipping and flipped loci are all tangent to the foliation. 
To prove Item (4), consider a step in the $K_{X}$-MMP, call it $f: Y \rightarrow W$ and let $\mathcal{F}_{Y}$ and $\mathcal{F}_{W}$ be the transformed foliations on $Y$ and $W$ respectively. We claim that if $f$ is a divisorial contraction, then $\operatorname{exc}(f)$ is foliation invariant. Indeed suppose not. By our above observation, $f$ contracts a divisor $E$ transverse to the foliation to a curve $C$ and such that the foliation restricted to $E$ must be tangent to the fibration $E \rightarrow C$. Let $F$ be a general fibre of $E \rightarrow C$. By Lemma 3.16 we know that

$$
0=K_{\mathcal{F}_{Y}} \cdot F=\left(K_{Y}+E\right) \cdot F
$$

a contradiction of the fact that $f$ is a $K_{Y}$-negative contraction.

Thus, all divisorial contractions in the MMP only contract invariant divisors and so by Lemma 12.5 we may conclude that Sing $X^{\prime}$ is indeed tangent to $\mathcal{F}^{\prime}$.

\section{Lemma $12.7 \mathcal{F}$ is not uniruled.}

Proof The proof of [29, Theorem 3.7] works equally well in the case where $X$ is singular.

Lemma 12.8 Suppose we have a morphism $f: X \rightarrow S$ where $S$ is a surface with klt singularities. Suppose furthermore that $K_{\mathcal{F}} \sim_{\mathbb{Q}, f} 0$ and the fibres of $f$ are tangent to $\mathcal{F}$.

Then $\kappa\left(K_{\mathcal{F}}\right)=0$.

Proof By Lemma 9.1, there is a foliation $\mathcal{G}$ on $S$ so that $\mathcal{F}=f^{-1} \mathcal{G}$, a pseudoeffective divisor $D$ and divisor $\Theta \geq 0$ such that $K_{\mathcal{F}} \sim_{\mathbb{Q}} f^{*}\left(K_{\mathcal{G}}+\Theta+D\right)$ and $(\mathcal{G}, \Theta)$ is lc. It suffices to show that $K_{\mathcal{G}}+\Theta$ is torsion.

Suppose for sake of contradiction that $D \neq 0$. Since $c_{1}\left(K_{\mathcal{G}}+\Theta\right)=-D$ it follows that $\mathcal{G}$ is uniruled, by Lemma 2.21. In particular, $\mathcal{G}$ is algebraically integrable, which implies the same holds for $\mathcal{F}$.

We may freely replace $(\mathcal{G}, \Theta)$ by an F-dlt modification and so we may assume without loss of generality that $S$ is klt. Thus, so we may apply Lemma 12.2 to conclude that $K_{\mathcal{G}}+\Theta$ is torsion.

Lemma 12.9 Suppose we have a morphism $f: X \rightarrow S$ where $S$ is a surface with klt singularities and $\kappa(S) \geq 0$. Suppose moreover that the fibres of $f$ are generically transverse to $\mathcal{F}$ and that for all $s \in S$, the fibre $f^{-1}(s)$ does not contain a divisor.

Then $\kappa\left(K_{\mathcal{F}}\right)=0$.

Proof Let $S^{\circ}=S \backslash \operatorname{Sing} S$ and let $X^{\circ}=f^{-1}\left(S^{\circ}\right)$.

The pull back of a pluri-canonical form on $S^{\circ}$ restricts to a non-zero form on the leaves of $\left.\mathcal{F}\right|_{X^{\circ}}$, i.e., we have a non-zero map

$$
H^{0}\left(S^{\circ}, \mathcal{O}_{S}\left(m K_{S}\right)\right) \rightarrow H^{0}\left(X^{\circ}, \mathcal{O}_{X^{\circ}}\left(m K_{\mathcal{F}}\right)\right)
$$


for all $m \geq 0$. Since the complement of $X^{\circ}$ in $X$ has codimension at least two, we see that $H^{0}\left(X^{\circ}, \mathcal{O}_{X^{\circ}}\left(m K_{\mathcal{F}}\right)\right) \cong H^{0}\left(X, \mathcal{O}_{X}\left(m K_{\mathcal{F}}\right)\right)$. By assumption, we have that $H^{0}\left(S, \mathcal{O}_{S}\left(m K_{S}\right)\right) \neq 0$ for some $m$ sufficiently divisible, and our result follows.

We will need the following definition, [43, Definition 8.1].

Definition 12.10 Let $X$ be a projective manifold and let $\mathcal{F}$ be a co-rank one foliation on $X$. Let $H_{1}, \ldots, H_{p}$ be $\mathcal{F}$-invariant hypersurfaces such that $\sum H_{i}$ is a normal crossings divisor. We say that $\mathcal{F}$ is of KLT type with respect to $H_{1}, \ldots, H_{p}$ if there exist rational numbers $0 \leq a_{i}<1$ such that

$$
N_{\mathcal{F}}^{*}+\sum a_{i} H_{i}
$$

is pseudo-effective.

We will also need the following theorem, due to Touzet.

Theorem 12.11 Let $X$ be a projective manifold and let $\mathcal{F}$ be a co-rank one foliation on $X$. Let $H_{1}, \ldots, H_{p}$ be $\mathcal{F}$-invariant hypersurfaces such that $\sum H_{i}$ is a normal crossings divisor. Suppose that $\mathcal{F}$ is of KLT type with respect to $H_{1}, \ldots, H_{p}$.

Then either

1. $\kappa\left(N_{\mathcal{F}}^{*}+\sum H_{i}\right)=v\left(N_{\mathcal{F}}^{*}+\sum H_{i}\right) \geq 0$ or

2. $\kappa\left(N_{\mathcal{F}}^{*}+\sum H_{i}\right)=-\infty, v\left(N_{\mathcal{F}}^{*}+\sum H_{i}\right)=1$ and there exists a holomorphic map $\Psi: X \rightarrow \mathfrak{h}$ where $\mathfrak{h}=\mathbb{D}^{n} / \Gamma$ is a quotient of a polydisc by an irreducible lattice $\Gamma \subset(\text { Aut } \mathbb{D})^{n}$ and $\mathcal{F}=\Psi^{-1} \mathcal{H}$ where $\mathcal{H}$ is one of the tautological foliations on $\mathfrak{h}$.

Proof This is [43, Theorem 6] and [43, Theorem 9.7].

We will also need the following classification theorem on surface foliations:

Theorem 12.12 Let $X$ be a normal projective surface and let $\mathcal{L}$ be a rank one foliation on $X$ with canonical foliation singularities. Suppose $c_{1}\left(K_{\mathcal{L}}\right)=0$.

Then there exists a finite cover $\tau: \widetilde{X} \rightarrow X$ and a birational morphism $\mu: \widetilde{X} \rightarrow Y$ such that if $\widetilde{\mathcal{L}}$ and $\mathcal{G}$ are the transformed foliations on $\tilde{X}$ and $Y$ respectively, then $\tau$ is ramified along $\widetilde{\mathcal{L}}$-invariant divisors and $\mu$ contracts

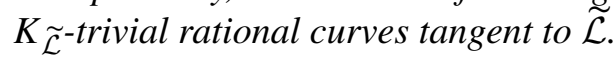

Moreover, one of the following holds:

1. $X=C \times E / G$ where $g(E)=1, C$ is a smooth projective curve, $G$ is a finite group acting on $C \times E$ and $\mathcal{G}$ is the foliation induced by the $G$-invariant fibration $C \times E \rightarrow C$;

2. $\mathcal{G}$ is a linear foliation on the abelian surface $Y$; 
3. $Y$ is a $\mathbb{P}^{1}$-bundle over an elliptic curve and $\mathcal{G}$ is transverse to the bundle structure and leaves at least one section invariant; or

4. $Y \cong \mathbb{P}^{2}, \mathbb{P}^{1} \times \mathbb{P}^{1}$ or $\mathbb{F}_{n}$ (the $n$-th Hirzebruch surface) and $\mathcal{G}$ admits at least 3 invariant rational curves. If $Y \cong \mathbb{P}^{1} \times \mathbb{P}^{1}$ or $\mathbb{F}_{n}$ then at least 2 of these invariant curves must be fibres of a $\mathbb{P}^{1}$-bundle structure on $Y$.

Proof This follows directly from [31, Theorem IV.3.6].

Lemma 12.13 Suppose that $\kappa(X) \geq 0$. Then $\kappa\left(K_{\mathcal{F}}\right)=0$.

Proof We have an equality of divisors $K_{\mathcal{F}}+N_{\mathcal{F}}^{*}=K_{X}$, in particular we see that $c_{1}\left(N_{\mathcal{F}}^{*}\right)=c_{1}\left(K_{X}\right)$.

If $\kappa(X)=3$, then $\kappa\left(N_{\mathcal{F}}^{*}\right)=3$, a contradiction of the BogomolovCastelnuovo-De Franchis inequality (see [21, Theorem 7.2] for the proof of this statement in the singular setting). Thus, we may assume that $\kappa(X) \leq 2$. Moreover, by Lemma 12.6 we may assume that $K_{X}$ is nef.

We distinguish two cases. We first assume that $\kappa\left(N_{\mathcal{F}}^{*}\right)=-\infty$. Let $\mu: Y \rightarrow$ $X$ be a $\log$ resolution of $X$ and let $E$ be the reduced divisor whose support coincides with the $\mu$-exceptional divisor and $\mathcal{G}$ be the pulled back foliation. By Lemma 12.6 Item (4), Sing $X$ is tangent to $\mathcal{F}$ and so $E$ is $\mathcal{G}$-invariant. Notice that $\mathcal{O}_{Y}\left(N_{\mathcal{G}}^{*}+E\right)$ is the saturation of the image of $\mu^{*} \mathcal{O}_{X}\left(N_{\mathcal{F}}^{*}\right)$ in $\Omega_{Y}^{1}(\log E)$. Since $\mu_{*} E=0$ and $\mu_{*} N_{\mathcal{G}}^{*}=N_{\mathcal{F}}^{*}$, we have that $\kappa\left(N_{\mathcal{G}}^{*}+E\right)=-\infty$.

Write $N_{\mathcal{G}}^{*}+E_{1} \sim_{\mathbb{Q}} \mu^{*} N_{\mathcal{F}}^{*}+E_{2}$ where $E_{i} \geq 0$ and is $\mu$-exceptional. We claim that $\left\lfloor E_{1}\right\rfloor=0$. Indeed, this is a local problem on both $X$ and $Y$, so perhaps shrinking both we may assume that $E_{1}=a E$ for some rational number $a>0$ so that $E_{1}$ consists of a single component and $N_{\mathcal{G}}^{*} \sim_{\mathbb{Q}} \mu^{*} N_{\mathcal{F}}^{*}-a E$. Consider the following commutative diagram

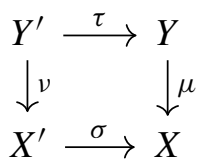

where $\sigma$ is the index one cover associated to $N_{\mathcal{F}}^{*}$. Let $\mathcal{F}^{\prime}$ be the pull back of $\mathcal{F}$ along $\sigma$ and let $\mathcal{G}^{\prime}$ be the pull back of $\mathcal{G}$ along $\tau$. Assume that the ramification index along $E$ is $r$. Since $X^{\prime}$ is klt, by [21] we have a morphism $v^{*}\left(\Omega_{X^{\prime}}^{[1]}\right) \rightarrow \Omega_{Y^{\prime}}^{1}$ and so

$$
N_{\mathcal{G}^{\prime}}^{*}=v^{*} N_{\mathcal{F}^{\prime}}^{*}+c E^{\prime},
$$

where $c \geq 0$. On the other hand, since $E^{\prime}$ is invariant

$$
N_{\mathcal{G}^{\prime}}^{*}=\tau^{*}\left(N_{\mathcal{G}}^{*}\right)+(r-1) E^{\prime} \sim_{\mathbb{Q}, v}(-r a+(r-1)) E^{\prime} .
$$


Thus, $-r a+(r-1)=c \geq 0$ which implies that $a \leq \frac{r-1}{r}<1$, as claimed.

Since $N_{\mathcal{G}}^{*}+E_{1}$ is pseudo-effective and $\left\lfloor E_{1}\right\rfloor=0$, it follows that $\mathcal{G}$ is a KLT type foliation.

Since $\kappa\left(N_{\mathcal{G}}^{*}+E\right)=-\infty$, Theorem 12.11 implies that $\nu\left(N_{\mathcal{G}}^{*}+E\right)=1$. Since $\mathcal{F}$ has canonical singularities, we may write $K_{\mathcal{G}} \equiv F$ where $F \geq 0$ is $\mu$-exceptional. Since $\left\lfloor E_{1}\right\rfloor=0$, it follows that

$$
K_{Y}+E=N_{\mathcal{G}}^{*}+E+K_{\mathcal{G}} \equiv N_{\mathcal{G}}^{*}+E+F
$$

has numerical dimension, and hence Kodaira dimension, equal to one, which in turn implies that $\kappa\left(K_{X}\right)=1$.

Let $f: X \rightarrow C$ be the Iitaka fibration associated to $K_{X}$. If $\mathcal{F}$ is the foliation induced by $f$ then we may conclude by Lemma 12.3. So suppose for sake of contadiction that the general fibre of $f$ is transverse to $\mathcal{F}$. We therefore get an exact sequence

$$
0 \rightarrow \mathcal{L} \rightarrow \mathcal{F} \rightarrow f^{*} T_{C} \otimes I_{Z} \rightarrow 0
$$

where $\mathcal{L}$ is the foliation by curves coming from the intersection of $\mathcal{F}$ and $T_{X / C}$ and $Z$ is a subscheme supported on $\operatorname{Sing} X \cup \operatorname{Sing} \mathcal{F}$ and on the locus where $\mathcal{F}$ is tangent to the fibres of $X \rightarrow C$.

Let $X_{p}$ be the fibre over $p \in C$, and let $\mathcal{F}_{p}$ be the foliation restricted to $X_{p}$. For general $p$ we see that $c_{1}\left(K_{\mathcal{F}_{p}}\right)=0$ and that $X_{p}$ is not uniruled. In particular, $\mathcal{F}_{p}$ is canonical. We claim that $N_{\mathcal{F}_{p}}^{*}$ is torsion for general $p$. Indeed, $K_{\mathcal{F}_{p}} \sim_{\mathbb{Q}} 0$ by Lemma 12.2 and $K_{X_{p}} \sim_{\mathbb{Q}} 0$ and so $N_{\mathcal{F}_{p}}^{*} \sim_{\mathbb{Q}} 0$. Thus, we may find a $\mathbb{Q}$-divisor $A$ on $X$ whose support is contained in fibres of $f$, but contains no fibre of $f$ and a divisor $B$ on $C$ such that $f^{*} B \sim_{\mathbb{Q}} N_{\mathcal{F}}^{*}+A$. However, $K_{X} \sim_{\mathbb{Q}} f^{*} H$ where $H$ is an ample $\mathbb{Q}$-divisor on $C$ and $K_{X} \equiv N_{\mathcal{F}}^{*}$. Thus, $A=0$ and $B \equiv H$. In particular, $B$ is ample and $\kappa\left(N_{\mathcal{F}}^{*}\right)=1$. It follows, by Lemma 12.14 , that $\mathcal{F}$ is algebraically integrable and so we may conclude by Lemma 12.3.

We now assume that $\kappa\left(N_{\mathcal{F}}^{*}\right) \geq 0$.

If $\kappa(X)=0$, then since $K_{X}$ is nef, it follows that both $N_{\mathcal{F}}^{*}$ and $K_{X}$, hence $K_{\mathcal{F}}$ are torsion, and we are done.

Suppose $\kappa(X) \geq 1$ and let $f: X \rightarrow B$ be the canonical map. By assumption, there exists $D \geq 0$ such that $N_{\mathcal{F}}^{*} \sim_{\mathbb{Q}} D$ and $D$ is numerically equivalent to $f^{*} H$ where $H \geq 0$ is ample. However, this implies that $D$ is actually supported on fibres of $f$, and is in fact equal to a sum of fibres (with the appropriate multiplicities), and so $D=f^{*} B$ for some ample divisor $B$. But this implies that $\kappa\left(N_{\mathcal{F}}^{*}\right) \geq 1$. By Lemma 12.14, it follows that $\mathcal{F}$ is in fact algebraically integrable and so we conclude by Lemma 12.3. 
Lemma 12.14 Let $X$ be a klt projective variety and let $\mathcal{F}$ be a co-rank one foliation on $X$ with non-dicritical singularities. Suppose that $\operatorname{Sing} X$ is tangent to $\mathcal{F}, N_{\mathcal{F}}^{*}$ is $\mathbb{Q}$-Cartier and $\kappa\left(N_{\mathcal{F}}^{*}\right)=1$.

Then $\mathcal{F}$ is algebraically integrable.

We thank the referee for suggesting the following proof of Lemma 12.14.

Proof Let $\pi: X^{\prime} \rightarrow X$ be a $\log$ resolution of $X$ and let $\mathcal{F}^{\prime}$ be the pulled back foliation. Let $E$ be the sum of all the $\pi$-exceptional divisors and observe that $E$ is $\mathcal{F}^{\prime}$-invariant.

By [16, Lemma 4.6] we may find effective $\pi$-exceptional divsiors $E_{1}$ and $E_{2}$ such that $E_{2}$ is $\mathcal{F}^{\prime}$-invariant and $\left\lfloor E_{2}\right\rfloor=0$ such that $\pi^{*} N_{\mathcal{F}}^{*}+E_{1} \sim_{\mathbb{Q}}$ $N_{\mathcal{F}^{\prime}}+E_{2}$. In particular, $\kappa\left(N_{\mathcal{F}^{\prime}}^{*}(E)\right)=1$. We may then apply [43, Lemma 9.2] to conclude.

Lemma 12.15 Let $X$ be a $\mathbb{Q}$-factorial projective threefold and let $\pi: X \rightarrow B$ be a fibration over a curve. Let $\mathcal{L}$ be a rank one foliation on $X$ tangent to the fibres of $X \rightarrow B$. Suppose that

1. $c_{1}\left(K_{\mathcal{L}}\right)=0$;

2. $\mathcal{L}$ is not uniruled; and

3. the general fibre of $\pi: X \rightarrow B$ does not admit a quasi-étale cover by an abelian surface.

Then $\kappa\left(K_{\mathcal{L}}\right)=0$.

Proof Observe first of all that we may assume that $\mathcal{L}$ has canonical singularities, otherwise $\mathcal{L}$ would be uniruled, a contradiction. $K_{\mathcal{L}_{p}}$.

Let $\mathcal{L}_{p}$ be the foliation restricted to $X_{p}$. For a general $p$ we have $\left.K_{\mathcal{L}}\right|_{X_{p}}=$

By Theorem 12.12, for a general fibre $X_{p}$, we may find a finite morphism $\tau_{p}: \widetilde{X}_{p} \rightarrow X_{p}$, ramified along foliation invariant divisors such that the pulled back foliation on $\widetilde{X}_{p}$ is generated by a global vector field. In particular, $\left.K_{\mathcal{L}}\right|_{X_{p}} \sim_{\mathbb{Q}} 0$. This implies that $K_{\mathcal{L}} \sim_{\mathbb{Q}} \sum a_{i} F_{i}$ where $a_{i}$ are rational numbers and $F_{i}$ are divisors supported on fibres of $f$, and are therefore in fact entire fibres (counted with multiplicity) since $c_{1}\left(K_{\mathcal{L}}\right)=0$. Thus, we may find a finite morphism $\sigma: \widetilde{X} \rightarrow X$ ramified only along divisors supported on fibres and such that if $\widetilde{\mathcal{L}}$ be the pulled back foliation on $\widetilde{X}$ then $K_{\widetilde{\mathcal{L}}}$ is Cartier. Since all the fibres of $X \rightarrow B$ are $\mathcal{L}$-invariant we have that $K_{\widetilde{\mathcal{L}}}=\sigma^{*} K_{\mathcal{L}}$. Thus we may freely replace $X$ and $\mathcal{L}$ by $\widetilde{X}$ and $\widetilde{\mathcal{L}}$ respectively and we may assume that $K_{\mathcal{L}}$ is Cartier.

In particular, around all points of $X$, we have that $\mathcal{L}$ is generated by a vector field $v$. Thus by [8] we may find a resolution of singularities $\mu: Y \rightarrow X$ by only blowing up in $\mathcal{L}$-invariant centres. Since $\mathcal{L}$ has canonical singularities this implies that $K_{\mathcal{L}_{Y}}=\mu^{*} K_{\mathcal{L}}$ where $\mathcal{L}_{Y}$ is the transformed foliation. Thus, 
we may replace $X$ and $\mathcal{L}$ by $Y$ and $\mathcal{L}_{Y}$ respectively and we may assume that $X$ is smooth.

We first handle the case where $\mathcal{L}_{p}$ is algebraically integrable for general $p$, and hence $\mathcal{L}$ is algebraically integrable. In this case we get a rational map $g: X \rightarrow S$ such that the fibres of $g$ induce $\mathcal{L}$. Again, by [8] we may find a resolution of singularities of $g, \mu: X^{\prime} \rightarrow X$, by blowing up in $\mathcal{L}$-invariant centres and let $f^{\prime}: X^{\prime} \rightarrow S$ be the induced map. Observe that $\mu^{*} K_{\mathcal{L}}=K_{\mathcal{L}^{\prime}}$ where $\mathcal{L}^{\prime}$ is the transformed foliation and we may argue as in Lemma 12.3 to show that $\kappa\left(K_{\mathcal{L}^{\prime}}\right)=0$ and so $\kappa\left(K_{\mathcal{L}}\right)=0$.

By Theorem 12.12 we may find a cover $\tau_{p}: \widetilde{X}_{p_{\mathcal{L}}} \rightarrow X_{p}$ ramified along invariant divisors and a birational contraction $\mu_{p}: \widetilde{X}_{p} \rightarrow Y_{p}$ such that $Y_{p}$ falls into one the types listed in Theorem 12.12. We now argue based on which case $Y_{p}$ in falls into. Note that, since the surfaces appearing in Cases (2), (3) and (4) of Theorem 12.12 are not deformation equivalent, we may consider the four cases separately. Observe also that in Case (2) that $\tau_{p}$ is an isomorphism, unless $\mathcal{L}_{p}$ is algebraically integrable. In Case (2) we also have that $\mu_{p}$ is an isomorphism since the foliation on $Y_{p}$ is smooth, hence terminal, and $c_{1}\left(K_{\tau_{p}^{-1} \mathcal{L}_{p}}\right)=0$.

In Case (1), we see that $\mathcal{L}$ is algebraically integrable and so we are done.

Case (2) does not occur by assumption.

In Case (3), we run a $K_{X}$-MMP over $B$, call it $\phi: X \rightarrow X^{\prime}$ and let $\mathcal{L}^{\prime}$ be the transformed foliation on $X^{\prime}$. Each step of this MMP is $\mathcal{L}$-trivial. Thus, it suffices to check that $\kappa\left(K_{\mathcal{L}^{\prime}}\right)=0$. A general fibre is uniruled, but not rationally connected, and so this MMP terminates in a Mori fibre space $g: X^{\prime} \rightarrow S$ over $B$ with $\operatorname{dim}(S)=2$. Let $h: S \rightarrow B$ be the induced morphism. As in the proof of Lemma 12.6 we see that $\phi$ only contracts curves tangent to $\mathcal{L}$. Note that since $K_{\mathcal{L}}$ is Cartier we also have that $K_{\mathcal{L}^{\prime}}$ is Cartier, and so $K_{\mathcal{L}^{\prime}}=g^{*} M$ where $M$ is a Cartier divisor on $S$ with $c_{1}(M)=0$.

By assumption, we know that the fibres of $g$ are generically not tangent to $\mathcal{L}^{\prime}$ and so we have a non-zero morphism

$$
d g:\left(g_{*} \mathcal{L}^{\prime}\right)^{* *} \cong M \rightarrow T_{S / B}
$$

Since $c_{1}(M)=0$ this immediately implies that for a general $p \in B$ that $\left.\left.M\right|_{S_{p}} \cong T_{S / B}\right|_{S_{p}}$, where $S_{p}$ is the fiber over $p$.

Since $\rho\left(X^{\prime} / S\right)=1$ and $K_{\mathcal{L}^{\prime}}$ is $g$-trivial, we see that $\left(g_{*} \mathcal{L}^{\prime}\right)^{* *}$ is a rank one reflexive sheaf and $c_{1}\left(\left(g_{*} \mathcal{L}^{\prime}\right)^{* *}\right)=0$. Thus, we have $\left(g_{*} \mathcal{L}^{\prime}\right)^{* *}=$ $T_{S / B}\left(-\sum a_{i} F_{i}\right)$ where $F_{i}$ are supported on fibres of $S \rightarrow B$.

Since $c_{1}\left(T_{S / B}\left(-\sum a_{i} F_{i}\right)\right)=0$ and noting that $T_{S / B}^{*}=K_{S / B}-R$ where $R$ is the ramification divisor of $h$, we have

$$
c_{1}\left(K_{S / B}+\sum a_{i} F_{i}-R\right)=0 .
$$


Thus,

$$
K_{S / B}+\sum a_{i} F_{i}-R \sim_{\mathbb{Q}} h^{*} M
$$

for some $\mathbb{Q}$-divisor $M$ on $B$ such that $c_{1}(M)=0$. We apply [6, Theorem 3.5] to conclude that $M \sim_{\mathbb{Q}} 0$ and hence $K_{\mathcal{L}}$ is torsion.

In Case (4), we proceed in a similar fashion to the above case with a few modifications. We first claim that we may assume without loss of generality that $\rho\left(X_{p}\right) \geq 2$. Since $\mathcal{L}_{p}$ is singular for a general $p$ it follows that there exists a component $\Sigma \subset$ Sing $\mathcal{L}$ such that $\Sigma$ dominates $B$. Let $b: \widetilde{X} \rightarrow X$ be a blow up centred in $\Sigma$ followed by a resolution of singularities which is a sequence of blow ups in foliation invariant centres, again which exists by [8]. Let $\widetilde{\mathcal{L}}$ be the pulled back foliation and observe that $K_{\widetilde{\mathcal{L}}}=b^{*} K_{\mathcal{L}}$. By construction we have $\rho\left(\widetilde{X}_{p}\right) \geq 2, c_{1}\left(K_{\widetilde{\mathcal{L}}}\right)=0$ and $\widetilde{\mathcal{L}}$ has canonical singularities. So we may freely replace $X$ by $\widetilde{X}$ as required.

For general $p$ let $D_{0, p}$ and $D_{\infty, p}$ denote two $\mathcal{L}_{p}$-invariant divisors which are fibres in a $\mathbb{P}^{1}$-fibration structure on $X_{p}$.

First, assume that there exist two divisors $D_{0}$ and $D_{\infty}$ on $X$ such that $D_{0} \cap X_{p}=D_{0, p}$ and $D_{\infty} \cap X_{p}=D_{\infty, p}$ for general $p$ and that if we run a $K_{X}$-MMP $\phi: X \rightarrow X^{\prime}$ over $B$, we terminate in a Mori fibre space $g: X^{\prime} \rightarrow S$ where $S$ is a surface, $D_{0}$ and $D_{\infty}$ are not contracted by $\phi$ and $g$ contracts the strict transforms of $D_{0}$ and $D_{\infty}$, call them $D_{0}^{\prime}$ and $D_{\infty}^{\prime}$. Arguing as above we see that $c_{1}\left(\left(g_{*} \mathcal{L}\right)^{* *}\right)=T_{S / B}\left(-\Sigma_{0}-\Sigma_{\infty}-\sum a_{i} F_{i}\right)$ where $\Sigma_{0}=g\left(D_{0}^{\prime}\right)$ and $\Sigma_{\infty}=g\left(D_{\infty}^{\prime}\right)$ are reduced divisors dominating $B$ and $F_{i}$ are supported on fibres of $S \rightarrow B$.

Since $c_{1}\left(T_{S / B}\left(-\Sigma_{0}-\Sigma_{\infty}-\sum a_{i} F_{i}\right)\right)=0$ and noting that $T_{S / B}^{*}=K_{S / B}-R$ where $R$ is the ramification divisor of $h$ we have

$$
c_{1}\left(K_{S / B}+\Sigma_{0}+\Sigma_{\infty}+\sum a_{i} F_{i}-R\right)=0 .
$$

Thus,

$$
K_{S / B}+\Sigma_{0}+\Sigma_{\infty}+\sum a_{i} F_{i}-R \sim_{\mathbb{Q}} h^{*} M .
$$

We again apply [6, Theorem 3.5] (see also [18, Theorem 1.3]) to conclude that $M \sim_{\mathbb{Q}} 0$ and hence $K_{\mathcal{L}}$ is torsion.

Thus, to conclude it suffices to arrange the existence of $D_{0}$ and $D_{\infty}$ and such a Mori fibre space structure. Let $p \in B$ be a general point. Observe that $\mathcal{L}$ is singular and so by assumption $\rho\left(X_{p}\right) \geq 2$. Then we may find a sufficiently small étale neighborhood $U$ of $p$ such that $X \times_{B} U$ admits divisors $D_{0}$ and $D_{\infty}$ as required and an MMP over $U$ terminating in the desired Mori fibre space structure. Thus, we may find a (possibly ramified) cover $\bar{B} \rightarrow B$ such 
that $\bar{X}=X \times{ }_{B} \bar{B}$ admits such an MMP over $\bar{B}$. Let $\overline{\mathcal{L}}$ be the pulled back foliation. Observe that $\sigma: \bar{X} \rightarrow X$ is ramified only along $\overline{\mathcal{L}}$-invariant divisors and so $K_{\overline{\mathcal{L}}}=\sigma^{*} K_{\mathcal{L}}$ and thus we may freely replace $X$ and $\mathcal{L}$ by $\bar{X}$ and $\overline{\mathcal{L}}$ ) respectively and our result follows.

Corollary 12.16 Suppose we have a morphism $f: X \rightarrow C$ where $C$ is a smooth curve of positive genus and suppose that the general fibre does not admit a quasi-étale cover by an abelian surface.

Then $\kappa\left(K_{\mathcal{F}}\right)=0$.

Proof By Lemma 12.3, we may assume that $\mathcal{F}$ is generically transverse to $f$. Let $\mathcal{L}$ be the foliation in curves tangent to both $\mathcal{F}$ and the fibration $f: X \rightarrow C$. We have an exact sequence

$$
0 \rightarrow \mathcal{L} \rightarrow \mathcal{F} \rightarrow\left(f^{*} T_{C}\right) \otimes I_{Z} \rightarrow 0,
$$

where $Z$ is supported on the components of fibres which are $\mathcal{F}$-invariant and on subvarieties of codimension at least 2. Thus, we have $K_{\mathcal{F}}=K_{\mathcal{L}}+f^{*} K_{C}+D$ where $D \geq 0$ is the codimension one part of $Z$.

By Lemma $12.7, \mathcal{F}$ is not uniruled and so we know that $K_{\mathcal{L}}$ is pseudoeffective. By assumption, $f^{*} K_{C}$ is nef, and since $K_{\mathcal{F}}$ is numerically trivial we must have $C$ is genus one and $D=0$. In particular, since $D=0$ we see that no component of a fibre of $f$ can be invariant under $\mathcal{F}$.

So $K_{\mathcal{L}} \sim_{\mathbb{Q}} K_{\mathcal{F}}$ and, in particular, it suffices to prove that $K_{\mathcal{L}}$ is torsion. Observe that $\mathcal{L}$ has canonical singularities above the generic point of $C$. We may now apply Lemma 12.15 to conclude.

Proposition 12.17 Suppose $\mathcal{F}$ has canonical singularities and $c_{1}\left(K_{\mathcal{F}}\right)=0$. Then $\kappa\left(K_{\mathcal{F}}\right)=0$.

Proof First, assume that $K_{X}$ is pseudo-effective. Then $\kappa\left(K_{X}\right) \geq 0$ and we apply Lemma 12.13 to conclude.

Now, assume that $K_{X}$ is not pseudo-effective. By Lemma 12.6, we may assume that $X$ admits a Mori fibre space $f: X \rightarrow B$. If $B$ is a surface with $\kappa(B) \geq 0$ or $f$ is tangent to $\mathcal{F}$, we may apply Lemmas 12.9 and 12.8 to conclude (note that since $f: X \rightarrow B$ is a Mori fibre space it follows that $f^{-1}(b)$ does not contain a divisor for any $b$ and that $B$ is klt since $X$ is). If $B$ is a rationally connected surface then $X$ is rationally connected and therefore it is immediate that $K_{\mathcal{F}}$ is torsion.

Otherwise, we may find a map $X \rightarrow C$ where $C$ is a curve. If $C$ is of positive genus then observe that if $F$ is a general fibre then $-K_{F} \neq 0$ and so it does not admit a quasi-étale cover by an abelian surface and we conclude by Corollary 12.16 . Otherwise $X$ is rationally connected and again it is immediate that $K_{\mathcal{F}}$ is torsion. 


\subsection{Proof of Theorem 12.1}

\section{Proof If $\Delta \neq 0$ or $\mathcal{F}$ is not canonical we apply Proposition 12.4. If $\Delta=0$ and $\mathcal{F}$ is canonical we apply Proposition 12.17 .}

Acknowledgements Both the authors were funded by EPSRC. We would like to thank J. $\mathrm{M}^{\mathrm{c}}$ Kernan, M. McQuillan, J. V. Pereira and R. Svaldi for many useful discussion. We are particularly grateful to the referees for carefully reading the paper and to help us to improve the presentation of the paper considerably. The first author would like to thank the National Center for Theoretical Sciences in Taipei and Professor J. A. Chen for their generous hospitality, where some of the work for this paper was completed.

Open Access This article is licensed under a Creative Commons Attribution 4.0 International License, which permits use, sharing, adaptation, distribution and reproduction in any medium or format, as long as you give appropriate credit to the original author(s) and the source, provide a link to the Creative Commons licence, and indicate if changes were made. The images or other third party material in this article are included in the article's Creative Commons licence, unless indicated otherwise in a credit line to the material. If material is not included in the article's Creative Commons licence and your intended use is not permitted by statutory regulation or exceeds the permitted use, you will need to obtain permission directly from the copyright holder. To view a copy of this licence, visit http://creativecommons.org/licenses/by/4.0/.

\section{References}

1. Araujo, C., Druel, S.: On Fano foliations. Adv. Math. 238, 70-118 (2013)

2. Araujo, C., Druel, S.: On codimension 1 del Pezzo foliations on varieties with mild singularities. Math. Ann. 360(3-4), 769-798 (2014)

3. Araujo, C., Druel, S.: Characterization of generic projective space bundles and algebraicity of foliations. Comment. Math. Helv. 94(4), 833-853 (2019)

4. Atiyah, M.F., Macdonald, I.G.: Introduction to Commutative Algebra. Addison-Wesley, London (1969)

5. Ambro, F.: Shokurov's boundary property. J. Differ. Geom. 67(2), 229-255 (2004)

6. Ambro, F.: The moduli $b$-divisor of an lc-trivial fibration. Compos. Math. 141(2), 385-403 (2005)

7. Birkar, C., Cascini, P., Hacon, C., $\mathbf{M}^{\mathrm{c}}$ Kernan, J.: Existence of minimal models for varieties of log general type. J. Am. Math. Soc. 23(2), 405-468 (2010)

8. Bierstone, E., Milman, P.D.: Canonical desingularization in characteristic zero by blowing up the maximum strata of a local invariant. Invent. Math. 128(2), 207-302 (1997)

9. Brunella, M.: Birational Geometry of Foliations. Monografías de Matemática. Instituto de Matemática Pura e Aplicada (IMPA), Rio de Janeiro (2000)

10. Cano, F.: Reduction of the singularities of codimension one singular foliations in dimension three. Ann. Math. (2) 160(3), 907-1011 (2004)

11. Cano, F., Cerveau, D.: Desingularization of nondicritical holomorphic foliations and existence of separatrices. Acta Math. 169(1-2), 1-103 (1992)

12. Cano, F., Mattei, J.: Hypersurfaces intégrales des feuilletages holomorphes. Ann. l'inst. Fourier 42(1-2), 49-72 (1992)

13. Corti, A.: 3-Fold flips after Shokurov. In: Corti, A. (ed.) Flips for 3-Folds and 4-Folds, pp. 13-40. Oxford University Press, Oxford (2005)

14. Campana, F., Păun, M.: Foliations with positive slopes and birational stability of orbifold cotangent bundles. Publ. Math. Inst. Hautes Études Sci. 129, 1-49 (2019) 
15. Druel, S., Ou, W.: Codimension one foliations with trivial canonical class on singular spaces II. arXiv:1912.07727 (2019)

16. Druel, S.: Codimension 1 foliations with numerically trivial canonical class on singular spaces. Duke Math. J. 170(1), 95-203 (2021)

17. Elkik, R.: Solutions d'équations à coefficients dans un anneau hensélien. Ann. Sci. École Norm. Sup. (4) 6(553-603), 1973 (1974)

18. Floris, E.: Inductive approach to effective b-semiampleness. Int. Math. Res. Not. IMRN 6, 1465-1492 (2014)

19. Fujita, T.: Semipositive line bundles. J. Fac. Sci. Univ. Tokyo Sect. IA Math. 30(2), 353-378 (1983)

20. Fujino, O.: Fundamental theorems for the log minimal model program. Publ. Res. Inst. Math. Sci. 47(3), 727-789 (2011)

21. Greb, D., Kebekus, S., Kovács, S., Peternell, T.: Differential forms on log canonical spaces. Publ. Math. Inst. Hautes Études Sci. 114, 87-169 (2011)

22. Grothendieck, A.: Éléments de géométrie algébrique. III. Inst. Hautes Études Sci. Publ. Math. 11, 91 (1961)

23. Hartshorne, R.: Algebraic Geometry. Springer, Berlin (1977)

24. Hartshorne, R.: Stable reflexive sheaves. Math. Ann. 254(2), 121-176 (1980)

25. Kollár, J., Mori, S.: Birational Geometry of Algebraic Varieties, Volume 134 of Cambridge Tracts in Mathematics. Cambridge University Press, Cambridge (1998)

26. Kollár, J.: Projectivity of complete moduli. J. Differ. Geom. 32(1), 235-268 (1990)

27. Kollár, J.: Kodaira's canonical bundle formula and adjunction. In: Flips for 3-Folds and 4Folds, Volume 35 of Oxford Lecture Series Mathematics and its Applications, pp. 134-162. Oxford University Press, Oxford (2007)

28. Kollár, J.: Singularities of the Minimal Model Program, Volume 200 of Cambridge Tracts in Mathematics. Cambridge University Press, Cambridge (2013). With a collaboration of Sándor Kovács

29. Loray, F., Pereira, J., Touzet, F.: Singular foliations with trivial canonical class. Invent. Math. 213(3), 1327-1380 (2018)

30. McQuillan, M.: Semi-stable reduction of foliations (2005). IHES/M/05/02

31. McQuillan, M.: Canonical models of foliations. Pure Appl. Math. Q. 4(3, Part 2), 877-1012 (2008)

32. Mendes, L.G.: Kodaira dimension of holomorphic singular foliations. Bol. Soc. Brasil. Mat. (N.S.) 31(2), 127-143 (2000)

33. $\mathrm{M}^{\mathrm{c}}$ Kernan, J., Prokhorov, Y.: Threefold thresholds. Manuscr. Math. 114(3), 281-304 (2004)

34. Nakayama, N.: The lower semicontinuity of the plurigenera of complex varieties. In: Algebraic Geometry, Sendai, 1985, Volume 10 of Adv. Stud. Pure Math., pp. 551-590. North-Holland, Amsterdam (1987)

35. Pereira, J.V.: Fibrations, divisors and transcendental leaves. J. Algebraic Geom. 15(1), 87-110 (2006). With an appendix by Laurent Meersseman

36. Prokhorov, Y.G., Shokurov, V.V.: Towards the second main theorem on complements. J. Algebraic Geom. 18(1), 151-199 (2009)

37. Raynaud, M.: Anneaux locaux henséliens. Lecture Notes in Mathematics, vol. 169. Springer, Berlin (1970)

38. Seidenberg, A.: Reduction of singularities of the differential equation $A d y=B d x$. Am. J. Math. 90, 248-269 (1968)

39. Shokurov, V.V.: Three-dimensional log perestroikas. Izv. Ross. Akad. Nauk Ser. Mat. 56(1), 105-203 (1992)

40. Shokurov, V.V.: Prelimiting flips. Proc. Steklov Inst. Math. 240, 82-219 (2003)

41. Spicer, C.: Higher dimensional foliated Mori theory. Compos. Math. 156(1), 1-38 (2020)

42. Temkin, M.: Functorial desingularization over $Q$ : boundaries and the embedded case. Isr. J. Math. 224(1), 455-504 (2018) 
43. Touzet, F.: On the structure of codimension 1 foliations with pseudoeffective conormal bundle. In: Foliation Theory in Algebraic Geometry, Simons Symp., pp. 157-216. Springer, Cham (2016)

Publisher's Note Springer Nature remains neutral with regard to jurisdictional claims in published maps and institutional affiliations. 